\title{
Correlation asymptotics for non-translation invariant lattice spin systems
}

\author{
Oliver Matte \\ Mathematisches Institut der Universität München \\ Theresienstraße 39, D-80333 München, Germany. \\ matte@mathematik. uni-muenchen.de
}

\begin{abstract}
We obtain asymptotic expressions for the Green kernels of certain non-translation invariant transition matrices using methods of semiclassical and microlocal analysis. Combined with a result by Bach and Møller this yields asymptotic formulas for the truncated two-point correlation functions of certain non-translation invariant lattice models of real-valued spins.
\end{abstract}

Keywords: Correlation asymptotics, Helffer-Sjöstrand formula, Fourier integral operator with complex-valued phase function, Finsler metric.

\section{Introduction}

This article is a further contribution to the study of correlation asymptotics for lattice systems of real-valued spins via the Witten-Laplacian approach [2, 3, 4, 13, 20, 21]. Like the authors did in the cited articles, we consider a lattice system at very small temperatures and assume that the on-site potentials have only one non-degenerated minimum and that the ferromagnetic pair interaction is sufficiently weak. In this situation one expects the truncated two-point correlation functions of the associated Gibbs measure to decay exponentially. In fact, in 2, 20, the leading asymptotics, as the temperature tends to zero and the distance on the lattice tends to infinity, of the exponential decay were computed under similar hypotheses. In [21] even a full asymptotic expansion has been obtained. The results of 2, 20, 21 hold for translation invariant systems on finite discrete tori and are uniform in the number of spins. (In 21 the thermodynamic limit of the derived expansions is discussed, too.) They provide a precise description of the Ornstein-Zernike behaviour which is actually well-known for translation invariant systems since a long time; see, e.g., [17. The starting point of the analysis of the correlation in [2, 3, 4, 20, 21] is the Helffer-Sjöstrand formula [7, 20]. which represents the truncated two-point correlation functions as matrix elements of the resolvent of a certain Witten-Laplacian on one-forms. If one works directly in the thermodynamic limit, or more precisely, if one considers a spin system on the lattice $\mathbb{Z}^{d}$ whose equilibrium distribution is given by a translation invariant tempered Gibbs measure, then the formula for the leading asymptotics derived in [2, 20, is still valid. This has been proved in [13] by replacing the WittenLaplacian by a supersymmetric Dirichlet operator and again using the Helffer-Sjöstrand formula. In [3, 4 the authors were able to drop the assumption of translation invariance in the study of the correlation asymptotics. (At the same time they were able to relax the conditions on the on-site potentials of 2, 20, 21.) They showed that, still for very small temperatures and for energy functionals with only one global minimum, the correlation asymptotics are determined by the Hessian of the energy functional at the global minimum. They derived this result for finite spin systems on graphs. Since all their results are again uniform in the number of spins, it is, however, easy to see that their methods work also in the infinite-dimensional case.

In this article we choose to work in the thermodynamic limit and our goal is to analyse the formal Hessian of the energy functional evaluated at the global minimum more precisely. The latter is an example of a transition operator in $\mathscr{L}\left(\ell^{2}\right)$. So the main result of this article is actually to develop a method to calculate the asymptotics of the Green kernel of a certain class of operators in $\mathscr{L}\left(\ell^{2}\right)$. Instead of considering the large distance asymptotics we fix two points in $\mathbb{R}^{d}$ and assume that they sit on lattices with vanishing lattice spacing, $\mathbb{Z}_{h}^{d}=\left\{h x: x \in \mathbb{Z}^{d}\right\}, h \in(0,1], d \geqslant 2$. At the same time we assume that the spatial variation of the interaction potentials is fixed, too. In 
this way we obtain a kind of continuum limit which permits to interprete the Hessian of our energy functional as a $h$-pseudodifferential operator on $\mathbb{R}^{d}$. We remark, however, that we introduce the continuum limit only for methodological reasons and that, in the limit, one would end up with a trivial theory. In order to analyse the Green kernel of the Hessian we therefore construct a parametrix for the $h$-pseudodifferential operator. This is essentially done via a geometric optics -or WKB- construction for the solution of a corresponding heat equation. Here we are lead to work with complex-valued symbols by the introduction of exponential weights. Consequently, we shall deal with Fourier integral operators with complex-valued phase functions [14, 15, 16, 19. Since the results of [15, 16] do not directly apply to our situation we show in detail how they can be suitably modified. In the end one verifies that the values of the Green kernel are, up to error terms of infinite order in $h$, given by the Fourier coefficients of the parametrix. Applying the method of stationary phase to these coefficients we find the leading asymptotics, as $h \rightarrow 0$, of the Green kernel. We obtain a formula which is similar to the expression known from the translation invariant case and which is determined by a certain Finsler structure naturally associated to the $h$-pseudodifferential operator. If we consider the correlation, for two points in $\mathbb{R}^{d}$ which are very close to each other, then we essentially observe again an Ornstein-Zernike behaviour. We remark that our results are closely related to those obtained in [8, 18, where the authors develop a symbol calculus on rescaled lattices and discuss Finsler structures associated to pseudodifferential operators in order to study the tunnel effect for a general class of difference operators.

The article is organized as follows. In Section 2 we state our hypotheses and our main result precisely. In Section 3 we determine the $h$-pseudodifferential operator mentioned above and conjugate it with exponential weights. In Section 4 we construct the appropriate exponential weight functions. In Section 5 we discuss the eikonal, or, time-dependent Hamilton-Jacobi equation proceeding along the lines of [15, 16. The transport equations are considered in Section 6. In Section 7 we construct the inverse of the formal Hessian of our energy functional at the global minimum and, finally, in Section 8 we calculate the leading asymptotics. The text is followed by three appendices: In Appendix $\mathrm{A}$ we prove that the results of 4 are applicable in our situation. In Appendix $B$ we present some elementary calculations which are used to reformulate the formula for the leading asymptotics in the translation invariant case. Some basic facts about almost analytic extensions are collected in Appendix C.

\section{Hypotheses and main results}

For every $h \in(0,1]$ and some $d \in \mathbb{N}, d \geqslant 2$, we consider a classical system of real-valued spins on the lattice $\mathbb{Z}_{h}^{d}:=\left\{h x: x \in \mathbb{Z}^{d}\right\}$, which is endowed with the Euclidean distance. A spin configuration is given by an element of $\mathbb{R}_{h}^{d}$ and denoted by $\sigma=\left(\sigma_{x}\right)_{x \in \mathbb{Z}_{h}^{d}}$. Furthermore, we write $\sigma_{\Upsilon}=\left(\sigma_{x}\right)_{x \in \Upsilon}$, for subsets $\Upsilon \subset \mathbb{Z}_{h}^{d}$. Our lattice spin model is determined by the choice of a heuristic energy functional of the system. The latter describes the interaction of the spins and is (formally) given by

$$
E(\sigma)=\sum_{x \in \mathbb{Z}_{h}^{d}} D\left(x, \sigma_{x}\right)+\frac{J}{2} \sum_{\substack{x, y \in \mathbb{Z}_{h}^{d}: \\ 0<|x-y| \leqslant h R}} W\left(\frac{x+y}{2}, \frac{x-y}{h}, \sigma_{x}-\sigma_{y}\right), \quad \sigma \in \mathbb{R}^{\mathbb{Z}_{h}^{d}}
$$

Here, the coupling constant $J>0$ will eventually be assumed to be sufficiently small. $R \in[1, \infty)$ is some arbitrarily large but finite interaction radius. We assume that the on-site potentials, $D$, and the pair interactions, $W$, fulfill the following

Hypothesis. 2.1 (i) It holds $D \in C^{\infty}\left(\mathbb{R}^{d} \times \mathbb{R}, \mathbb{R}\right)$ and there exist $C, \widetilde{C}, \varkappa>0$ and $L>2$ such 
that, for all $(x, \theta) \in \mathbb{R}^{d} \times \mathbb{R}$,

$$
\begin{aligned}
\left|\partial_{\theta}^{\nu} D(x, \theta)\right| & \leqslant C\langle\theta\rangle^{L-\nu}, \quad \nu=0,1,2, \\
\partial_{\theta}^{2} D(x, \theta) & \geqslant-C, \\
\left|\partial_{\theta}^{2} D(x, \theta)-\partial_{\theta}^{2} D(x, 0)\right| & \leqslant C\left|\partial_{\theta} D(x, \theta)\right|, \\
\partial_{\theta} D(x, \theta) \operatorname{sgn}(\theta) & \geqslant \widetilde{C} \max \left\{|\theta|^{1+\varkappa},|\theta|\right\} .
\end{aligned}
$$

For $\theta=0$, we have $\partial_{\theta}^{2} D(\cdot, 0) \in C_{b}^{\infty}\left(\mathbb{R}^{d}\right)$ and

$$
2 \inf _{x \in \mathbb{R}^{d}} \partial_{\theta}^{2} D(x, 0)>\sup _{x \in \mathbb{R}^{d}} \partial_{\theta}^{2} D(x, 0) .
$$

(ii) For all $\ell \in \mathbb{Z}^{d}, 0<|\ell| \leqslant R$, we have $W(\cdot, \ell, \cdot \cdot) \in C^{\infty}\left(\mathbb{R}^{d} \times \mathbb{R}, \mathbb{R}\right)$ and, for $(x, \theta) \in \mathbb{R}^{d} \times \mathbb{R}$,

$$
\begin{aligned}
W(x, \ell, \theta) & =W(x,-\ell, \theta)=W(x, \ell,-\theta), \\
W(x, \ell, \theta) & \geqslant 0, W(x, \ell, 0)=0 \\
|\ell|=1 & \Rightarrow \partial_{\theta}^{2} W(x, \ell, 0) \geqslant 1 \\
\sup _{(x, \theta) \in \mathbb{R}^{d+1}}\left\{\left|\partial_{\theta}^{2} W(x, \ell, \theta)\right|\right. & \left.+\left|\partial_{\theta}^{3} W(x, \ell, \theta)\right|\right\}<\infty .
\end{aligned}
$$

Finally, $\partial_{\theta}^{2} W(\cdot, \ell, 0) \in C_{b}^{\infty}\left(\mathbb{R}^{d}\right)$.

Remark. 2.2 The estimates (11)-(9) are required in order to apply the results of [1, 4, 13]. We assume that $\partial_{\theta}^{2} D(\cdot, 0)$ and $\partial_{\theta}^{2} W(\cdot, \ell, 0)$ are elements of $C_{b}^{\infty}\left(\mathbb{R}^{d}\right)$, because this will allow us to interprete the formal Hessian of $E$ at zero as a h-pseudodifferential operator. We notice that Hypothesis 2.1 implies that, for every $x \in \mathbb{Z}_{h}^{d}$, the on-site potential $D(x, \cdot)$ has only one local minimum, namely a non-degenerate one at zero, and tends to infinity, as $\theta \rightarrow \pm \infty$, faster than any quadratic polynomial. We also point out that $D(x, \cdot)$ does not have to be convex. (6) and (7) imply that $\partial_{\theta}^{2} W(x, \ell, 0) \geqslant 0$, for all $x \in \mathbb{R}^{d}$ and $\ell \in \mathbb{Z}^{d}, 0<|\ell| \leqslant R$.

Example. 2.3 We let $m \in \mathbb{N}, m \geqslant 2$, and suppose that there are functions $c_{0}, c_{1}, \ldots, c_{2 m} \in$ $C_{b}^{\infty}\left(\mathbb{R}^{d}\right)$ such that $\inf c_{2 m}>0,2 \inf c_{2}>\sup c_{2}$,

$$
D(x, \theta)=c_{2 m}(x) \theta^{2 m}+\ldots+c_{1}(x) \theta+c_{0}(x), \quad x \in \mathbb{R}^{d}, \theta \in \mathbb{R},
$$

and $\partial_{\theta} D(x, \theta)=0 \Leftrightarrow \theta=0$, for $x \in \mathbb{R}^{d}$. Furthermore, we suppose that $W(\cdot, \ell, \cdot \cdot) \equiv 0$, for $|\ell| \neq 1$, and that there is some $w \in C_{b}^{\infty}\left(\mathbb{R}^{d}\right), w \geqslant 1 / 2$, such that

$$
W(x, \ell, \theta)=w(x) \theta^{2},
$$

for $|\ell|=1, x \in \mathbb{R}^{d}$, and $\theta \in \mathbb{R}$. Then Hypothesis 2.1 is fulfilled. If $c_{0}, \ldots, c_{2 m}$ and $w$ are constant, we obtain examples of translation invariant models, which arise in lattice approximations of Euclidean quantum field theories of polynomial type and are known as " $P(\varphi)_{d}$-models".

Next, we recall the notion of a tempered Gibbs measure for the spin system under consideration. To begin with we introduce, for every finite subset $\Upsilon \subset \mathbb{Z}_{h}^{d}$, a (well-defined) local energy functional with boundary condition $\omega \in \mathbb{R}^{\mathbb{Z}_{h}^{d}}$,

$$
\begin{aligned}
E_{\Upsilon}\left(\sigma_{\Upsilon} \mid \omega_{\Upsilon^{c}}\right):= & \sum_{x \in \Upsilon} D\left(x, \sigma_{x}\right)+\frac{J}{2} \sum_{\substack{x, y \in \Upsilon \\
0<|x-y| \leqslant h R}} W\left(\frac{x+y}{2}, \frac{x-y}{h}, \sigma_{x}-\sigma_{y}\right) \\
& +J \sum_{\substack{x \in \Upsilon, y \in \Upsilon^{c}: \\
|x-y| \leqslant h R}} W\left(\frac{x+y}{2}, \frac{x-y}{h}, \sigma_{x}-\omega_{y}\right), \quad \sigma_{\Upsilon} \in \mathbb{R}^{\Upsilon} .
\end{aligned}
$$


We further introduce a local specification, which is a family of stochastic kernels indexed by all finite subsets $\Upsilon \subset \mathbb{Z}_{h}^{d}$ and depending on the parameters $h \in(0,1]$ and the so-called inverse temperature $\beta \in[1, \infty)$. Given a tempered boundary condition, $\omega \in \bigcup_{\alpha \in \mathbb{N}} \ell_{-\alpha}^{2}$, where $\ell_{\alpha}^{2}=\{\sigma \in$ $\left.\mathbb{R}_{h}^{\mathbb{Z}_{h}}:\left(\langle x\rangle^{\alpha} \sigma_{x}\right)_{x} \in \ell_{\mathbb{R}}^{2}\left(\mathbb{Z}_{h}^{d}\right)\right\}$, their values are defined by

$$
\mu_{\beta, \Upsilon, h}(A, \omega):=\frac{1}{\mathscr{Z}_{\Upsilon}(\beta, \omega, h)} \int_{\mathbb{R}^{\Upsilon}} \mathbb{1}_{A}\left(\sigma_{\Upsilon} \mid \omega_{\Upsilon^{c}}\right) \exp \left(-\beta E_{\Upsilon}\left(\sigma_{\Upsilon} \mid \omega_{\Upsilon^{c}}\right)\right) d \sigma_{\Upsilon},
$$

for $A \in \mathfrak{B}\left(\mathbb{R}_{h}^{d}\right)$, where $\mathfrak{B}\left(\mathbb{R}_{h}^{d}\right)$ denotes the Borel- $\sigma$-algebra defined by the product topology on $\mathbb{R}^{\mathbb{Z}_{h}^{d}}$. The partition function, $\mathscr{Z}_{\Upsilon}(\beta, \omega, h)$, is a normalization factor. For $\omega \in \mathbb{R}_{h}^{d} \backslash\left(\bigcup_{\alpha \in \mathbb{N}} \ell_{-\alpha}^{2}\right)$, we set $\mu_{\beta, \Upsilon, h}(A, \omega)=0$. A probability measure, $\mu_{\beta, h}$, on the Borel sets of $\mathbb{R}_{h}^{d}$ is called a Gibbs measure (for the model determined by $E$ ), iff it satisfies the Dobrushin-Lanford-Ruelle equilibrium equations,

$$
\int_{\mathbb{R}^{\mathbb{Z}_{h}^{d}}} \mu_{\beta, h, \Upsilon}(A, \omega) d \mu_{\beta, h}(\omega)=\mu_{\beta, h}(A), \quad \Upsilon \subset \mathbb{Z}_{h}^{d} \text { finite, } A \in \mathfrak{B}\left(\mathbb{R}^{\mathbb{Z}_{h}^{d}}\right) .
$$

A Gibbs measure $\mu_{\beta, h}$ is called tempered iff $\mu_{\beta, h}\left(\ell_{-\alpha}^{2}\right)=1$, for some $\alpha>d / 2$.

Under Hypothesis 2.1 the results of [1] are applicable and ensure, for all $\beta \in[1, \infty), J>0$, and $h \in(0,1]$, the existence of a convex set, $\mathscr{G}(\beta, J, h)$, of tempered Gibbs measures. This convex set contains its extreme points, which are called pure, tempered Gibbs measures. We fix such a pure, tempered Gibbs measure, $\mu_{\beta, h}$, in the following, let $\mathrm{p}$ denote the projection onto the constant functions in $\mathscr{H}^{0}:=L^{2}\left(\mu_{\beta, h}\right)$, and set $\mathrm{p}^{\perp}=\mathbb{1}-\mathrm{p}$. We are interested in the asymptotic behaviour, as $h \rightarrow 0$, of the truncated two-point correlation functions,

$$
\operatorname{Cor}_{\beta, h}\left(\sigma_{x} ; \sigma_{y}\right)=\left\langle\sigma_{x} \mid \mathrm{p}^{\perp} \sigma_{y}\right\rangle_{\mathscr{H} 0}
$$

for fixed $x, y \in \mathbb{Z}_{h}^{d}$, at very large inverse temperatures $\beta$. In this situation we expect the spin configurations to be localized with high probability near the constant configuration $(0)_{x \in \mathbb{Z}_{h}^{d}}$, because all on-site potentials $D(x, \cdot)$ have a unique minimum at zero. In fact, it turns out that the asymptotic behaviour of $\operatorname{Cor}_{\beta, h}$ is determined by the formal Hessian of $E$ at zero, $E^{\prime \prime}(0) \in \mathscr{L}\left(\ell^{2}\left(\mathbb{Z}_{h}^{d}\right)\right)$, where $E^{\prime \prime}(\sigma)$ is given by

$$
\left(E^{\prime \prime}(\sigma)\right)_{x y}:=\partial_{\sigma_{x}} \partial_{\sigma_{y}} E_{\{y\}}\left(\sigma_{y} \mid \sigma_{\{y\}^{c}}\right)=\left(E^{\prime \prime}(\sigma)\right)_{y x},
$$

for $x, y \in \mathbb{Z}_{h}^{d}$ and $\sigma \in \mathbb{R}^{\mathbb{Z}_{h}^{d}}$. Here the second equality follows from (6), which we also use to derive the following explicit formulas,

$$
\left(E^{\prime \prime}(0)\right)_{x y}= \begin{cases}U_{h}(x), & x=y \\ -V\left(\frac{x+y}{2}, \frac{x-y}{h}\right), & 0<|x-y| \leqslant h R \\ 0, & |x-y|>h R\end{cases}
$$

for $x, y \in \mathbb{Z}_{h}^{d}$, where

$$
\begin{aligned}
U_{h}(x) & :=D_{\theta \theta}^{\prime \prime}(x, 0)+J \sum_{0<|\ell| \leqslant R} W_{\theta \theta}^{\prime \prime}\left(x+\frac{h \ell}{2}, \ell, 0\right), \\
V(x, \ell) & :=J W_{\theta \theta}^{\prime \prime}(x, \ell, 0)
\end{aligned}
$$

for $x \in \mathbb{R}^{d}, 0<|\ell| \leqslant R$. We state the precise relationship between $E^{\prime \prime}(0)$ and $\operatorname{Cor}_{\beta, h}$ in the following theorem which is essentially due to Bach and Møller [4]. The latter authors actually consider finite lattice systems uniformly in the number of spins. Using the estimates obtained in [13] it is, however, easy to see that their results extend to our situation.

We remark that, due to (5) and (9), we know that $E^{\prime \prime}(0)$ is continuously invertible, if $J>0$ is sufficiently small. 
Theorem. 2.4 Assume that Hypothesis [2.1 is fulfilled and let $\mu_{\beta, h} \in \mathscr{G}(\beta, J, h)$ be pure. Then there exist $\beta_{0} \geqslant 1, J_{0}>0$, and $C>0$, such that, for all $\beta \geqslant \beta_{0}, J \in\left[0, J_{0}\right]$, and $h \in(0,1]$,

$$
\frac{1-C / \sqrt{\beta}}{\beta}\left(E^{\prime \prime}(0)^{-1}\right)_{x y}^{1+\frac{C}{\sqrt{\beta}}} \leqslant \operatorname{Cor}_{\beta, h}\left(\sigma_{x} ; \sigma_{y}\right) \leqslant \frac{1+C / \sqrt{\beta}}{\beta}\left(E^{\prime \prime}(0)^{-1}\right)_{x y}^{1-\frac{C}{\sqrt{\beta}}} .
$$

ProOF: The claim follows from the results obtained in [4] together with the estimates derived in [13. We remark that all statements and estimates of these articles when applied to our situation hold uniformly in $h$ since all constants in Hypothesis 2.1 are $h$-independent. We present the details in Appendix A

So, our aim will be to calculate the leading asymptotics, as $h \rightarrow 0$, of the matrix elements $\left(E^{\prime \prime}(0)^{-1}\right)_{x y}$, for fixed $x, y \in \mathbb{Z}_{h}^{d}$. The main point here is that we do not assume the spin system to be invariant with respect to translations on the lattice. In the translation invariant case, that is, if $D$ and $W$ do not depend on the first variable $x$, the asymptotic behaviour of the two-point correlation functions is well-known from Ornstein-Zernike theory; see, e.g. [17]. We recall the precise result in Theorem 2.5. In the translation invariant case we can actually set $h$ equal to one by a scaling transformation and consider the large distance asymptotics instead. The detailed form of the leading asymptotics (16) given in Theorem 2.5 has been derived in 2, 20, 21, for translation invariant systems on finite discrete tori. As a starting point the authors used the Helffer-Sjöstrand formula for the correlation which involves a certain Witten-Laplacian associated to the lattice model. The validity of (16) for pure, translation invariant tempered Gibbs measures has been proven in 13 again using a Helffer-Sjöstrand formula. In the infinite-dimensional setting of the latter paper the Witten-Laplacian is replaced by a certain supersymmetric Dirichlet operator.

Before we state the next theorems we have to introduce some notation. First, we define a Hamilton function, $H \in C^{\infty}\left(T^{*} \mathbb{R}^{d}, \mathbb{R}\right)$, by

$$
H(x, p):=\sum_{\substack{\ell \in \mathbb{Z}^{d}: \\ 0<|\ell| \leqslant R}} V(x, \ell) e^{-\langle\ell \mid p\rangle}-U(x),
$$

for $x \in \mathbb{R}^{d}, p \in \mathbb{R}_{d}$, where $V$ is given by (13) and

$$
U(x):=D_{\theta \theta}^{\prime \prime}(x, 0)+\sum_{\substack{\ell \in \mathbb{Z}^{d}: \\ 0<|\ell| \leqslant R}} V(x, \ell) .
$$

For each $x \in \mathbb{R}^{d}, H(x, \cdot)$ is strictly convex and even and $H(x, 0)<0$, provided $J>0$ is small enough. It therefore makes sense to define the polar body

$$
\mathbb{k}_{x}^{*}:=\left\{p \in \mathbb{R}_{d} \mid H(x, p) \leqslant 0\right\},
$$

which is a strictly convex set that is symmetric about the origin and has a smooth boundary. Moreover, we let

$$
F(x, v):=\sup \left\{\langle p \mid v\rangle \mid p \in \mathbb{k}_{x}^{*}\right\}, \quad v \in T_{x} \mathbb{R}^{d}=\mathbb{R}^{d},
$$

denote the support function of $\mathbb{k}_{x}^{*}$. For $v \neq 0$, we have the explicit formula

$$
F(x, v)=\langle p(x, v) \mid v\rangle,
$$

where $p(x, v) \in \mathbb{E}_{x}:=\partial \mathbb{k}_{x}^{*}$ is the unique point, where the exterior normal field on $\mathbb{F}_{x}$ points in the direction of $v$. In particular, $F$ is smooth on the slit tangent bundle $\dot{T} \mathbb{R}^{d}=T \mathbb{R}^{d} \backslash\left(\mathbb{R}^{d} \times\{0\}\right)$ and absolutely homogenous of degree one in $v$, i.e. $F(x, \lambda v)=|\lambda| F(x, v)$, for $\lambda \in \mathbb{R},(x, v) \in T \mathbb{R}^{d}$. We also introduce the positive definite $d \times d$-matrix $G(x, v)$ with entries

$$
G(x, v)_{i j}:=F(x, v) F_{v^{i} v^{j}}^{\prime \prime}(x, v)+F_{v^{i}}^{\prime}(x, v) F_{v^{j}}^{\prime}(x, v), \quad(x, v) \in \dot{T} \mathbb{R}^{d} .
$$

$G_{i j}$ is homogenous of degree zero in $v$, i.e. $G_{i j}(x, \lambda v)=G_{i j}(x, v)$, for $\lambda \in \mathbb{R},(x, v) \in \dot{T} \mathbb{R}^{d}$. Finally, we let $H_{p p}^{\prime \prime}(x, p)^{\perp}$ denote the Hessian of $H(x, \cdot)$ at $p \neq 0$ restricted to the orthogonal complement of $\nabla_{p} H(x, p)$ in $\mathbb{R}_{d}$. In the translation invariant setting of the next theorem the functions $H, F, p$, and $G$ are $x$-independent, whence we drop the reference to $x$ in the notation of its statement. 
Theorem. 2.5 Assume that Hypothesis 2.1 is fulfilled, that $J>0$ is sufficiently small, and assume additionally that $D(x, \theta)$ and $W(x, \ell, \theta)$ are constant in $x$. Then, for sufficiently large $\beta$, there is a unique tempered Gibbs measure determined by $D$ and $W$. It is invariant with respect to translations on the lattice and its truncated two-point correlation functions fulfill

$$
\begin{aligned}
\operatorname{Cor}_{\beta, h}\left(\sigma_{x} ; \sigma_{y}\right)= & \frac{1}{\beta} \frac{\left|\nabla_{p} H(p(x-y))\right|^{\frac{d-3}{2}}}{\sqrt{\operatorname{det} H_{p p}^{\prime \prime}(p(x-y))^{\perp}}} \frac{1+\mathcal{O}\left(\frac{h}{|x-y|}\right)+\mathcal{O}\left(\beta^{-1 / 2}\right)}{\left(2 \pi \frac{|x-y|}{h}\right)^{\frac{d-1}{2}}} \\
& \times \exp \left(-\left(1+\mathcal{O}\left(\beta^{-1 / 2}\right)\right) F\left(\frac{x-y}{h}\right)\right) \\
= & \frac{1}{\beta} \frac{\sqrt{\operatorname{det} G(x-y)}}{\left\langle p(x-y) \mid \nabla_{p} H(p(x-y))\right\rangle} \frac{1+\mathcal{O}\left(\frac{h}{|x-y|}\right)+\mathcal{O}\left(\beta^{-1 / 2}\right)}{\left(2 \pi F\left(\frac{x-y}{h}\right)\right)^{\frac{d-1}{2}}} \\
& \times \exp \left(-\left(1+\mathcal{O}\left(\beta^{-1 / 2}\right)\right) F\left(\frac{x-y}{h}\right)\right),
\end{aligned}
$$

as $\beta \rightarrow \infty$ and $|x-y| / h \rightarrow \infty, x, y \in \mathbb{Z}_{h}^{d}$.

Proof: Using (16), which is derived in the above mentioned papers, and some elementary linear algebra we deduce Formula (17) in Appendix B.

In the following theorem we state the main result of this article. To formulate it, we introduce the Finsler distance associated to $F$,

$$
d_{F}(x, y)=\inf _{q} \int F(q, \dot{q}), \quad x, y \in \mathbb{R}^{d},
$$

where the infimum is taken over all piecewise smooth curves $q:[0, \tau] \rightarrow \mathbb{R}^{d}$ such that $\tau>0, q(0)=$ $y, q(\tau)=x$. It turns out that the infimum is always attained by at least one (smooth) geodesic from $y$ to $x$. Since $F$ is absolutely homogenous of degree one in $v$ any reparametrization of a minimizing geodesic yields again a minimizing geodesic and $d_{F}$ is symmetric. We can always reparametrize any minimizing geodesic in such a way that we obtain the projection of a Hamiltonian trajectory defined by $H$ which runs in the level surface $\mathbb{E}:=H^{-1}(\{0\})$. If there is, up to reparametrization, a unique minimizing geodesic from $y$ to $x$ we denote its initial (resp. end) velocity and momentum in the just mentioned special parametrization by $v_{y}$ and $p_{y}$ (resp. $v_{x}$ and $p_{x}$ ). We remark that we have $\left\langle p_{x} \mid v_{x}\right\rangle>0$ and $v_{x}=\nabla_{p} H\left(x, p_{x}\right)$ and analogous statements for $y$. As in Riemannian geometry one may define the notion of a conjugate point for $x$ and $y$ with respect to $F$; see Section 4 below.

Finally, we set $h_{n}:=1 / 2^{n}, n \in \mathbb{N}$. If $N \in \mathbb{N}$ and $x \in \mathbb{Z}_{h_{N}}^{d}$, then of course $x \in \mathbb{Z}_{h_{n}}^{d}$, for all integers $n \geqslant N$.

Theorem. 2.6 Assume that Hypothesis 2.1 is fulfilled and that $J>0$ is sufficiently small. Let $N \in \mathbb{N}, x, y \in \mathbb{Z}_{h_{N}}^{d}$, and assume that, up to reparametrization, there exists a unique geodesic minimizing Finslerian arc length from $y$ to $x$ and that $x$ and $y$ are not conjugate to each other. (This assumption is always fulfilled, for fixed $y$, provided $x$ is sufficiently close to $y$.) Then

$$
\begin{aligned}
\left(E^{\prime \prime}(0)^{-1}\right)_{x y}= & \frac{\sqrt[4]{\operatorname{det}\left(G\left(x, v_{x}\right) G\left(y, v_{y}\right)\right)}}{\Delta(x, y) \sqrt{\left\langle p_{x} \mid v_{x}\right\rangle\left\langle p_{y} \mid v_{y}\right\rangle}} \frac{\exp \left(-d_{F}(x, y) / h_{n}\right)}{\left(2 \pi d_{F}(x, y) / h_{n}\right)^{\frac{d-1}{2}}} \\
& +\mathcal{O}\left(h_{n}^{\frac{d+1}{2}}\right) \exp \left(-d_{F}(x, y) / h_{n}\right),
\end{aligned}
$$

as $n \rightarrow \infty$, where

$$
\Delta(x, y)=1+\mathcal{O}\left(d_{F}(x, y)^{1 / 2}\right), \quad \text { as } x \rightarrow y .
$$

PROOF: The theorem is proved by combining Propositions 4.5(ii), 7.1, 8.1 \& 8.2 and Equations (109) \& (115) . 
Remark. 2.7 The function $\Delta(x, y)$ can be expressed explicitely in terms of transversal Jacobi fields along the unit speed geodesic, $q$, from $y$ to $x$; see Proposition 8.2 and the remarks preceeding it. If the geodesics emanating from $y$ are dispersing along $q$, then $\Delta(x, y)$ increases when $d_{F}(x, y)$ gets large. If they are bunching together, $\Delta(x, y)$ decreases and vanishes, if $x$ is conjugate to $y$. If the flag curvature [5] happens to be zero along $q$, then $\Delta(x, y)$ is equal to one.

\section{A related $h$-pseudodifferential operator}

The basic idea underlying our analysis is the fact that, for every $f \in \ell^{2}\left(\mathbb{Z}_{h}^{d}\right)$, the expression (recall (11)-(13) )

$$
\left(E^{\prime \prime}(0) f\right)(x)=U_{h}(x) f(x)-\sum_{\substack{y \in \mathbb{Z}_{h}^{d}: \\ 0<|x-y| \leqslant h R}} V\left(\frac{x+y}{2}, \frac{x-y}{h}\right) f(y),
$$

can be interpreted as the image of any $\tilde{f} \in C^{\infty}\left(\mathbb{R}^{d}\right)$ with $\left.\tilde{f}\right\rceil_{\mathbb{Z}_{h}^{d}}=f$ under a $h$-pseudodifferential operator evaluated at the point $x \in \mathbb{Z}_{h}^{d}$. Before we explain this correspondence in detail, we fix some notation and recall some general facts.

For our purposes it is sufficient to consider only bounded symbols: Let $\Omega$ be an open subset of $\mathbb{R}^{d}, \mathbb{R}^{d} \times \mathbb{R}_{d}$, or $[0, \infty) \times \mathbb{R}^{d} \times \mathbb{T}^{d}$ etc., where $\mathbb{T}^{d}:=(\mathbb{R} / 2 \pi \mathbb{Z})^{d}$ is the $d$-dimensional torus. Then we write $f \in S_{b}(\Omega)$, for a function $f: \Omega \times(0,1] \rightarrow \mathbb{C}$, iff $f(\cdot ; h) \in C^{\infty}(\Omega)$, for all $h \in(0,1]$, and there is some $h_{0} \in(0,1]$ such that, for all multi-indices $\alpha$,

$$
\sup _{(\omega, h) \in \Omega \times\left(0, h_{0}\right]}\left|\partial_{\omega}^{\alpha} f(\omega ; h)\right|<\infty .
$$

We recall that the Weyl quantization, $\mathrm{Op}_{h}^{W}(a)$, of any symbol $a \in S_{b}\left(\mathbb{R}^{d} \times \mathbb{R}_{d}\right)$ is determined by the oscillatory integrals

$$
\mathrm{Op}_{h}^{W}(a) \tilde{f}(x)=\int e^{i\langle\xi \mid x-y\rangle / h} a\left(\frac{x+y}{2}, \xi\right) \tilde{f}(y) \frac{d y d \xi}{(2 \pi h)^{d}}, \quad \tilde{f} \in \mathscr{S}\left(\mathbb{R}^{d}\right), \quad x \in \mathbb{R}^{d},
$$

where $\mathscr{S}\left(\mathbb{R}^{d}\right)$ is the space of Schwartz test functions on $\mathbb{R}^{d}$. The possibility to regard (18) as the action of a pseudodifferential operator is a consequence of the following: If $a \in S_{b}\left(\mathbb{R}^{d} \times \mathbb{R}_{d}\right)$ is a symbol which is $(2 \pi \mathbb{Z})^{d}$-periodic in $\xi$, then its Weyl quantization has the distribution kernel

$$
K_{a}(x, y)=\left(\mathscr{F}_{h}^{-1} a\right)\left(\frac{x+y}{2}, x-y\right)=h^{d} \sum_{z \in \mathbb{Z}_{h}^{d}} \widehat{a}\left(\frac{x+y}{2}, z\right) \delta_{z}(y-x),
$$

where $\left(\mathscr{F}_{h} u\right)(\xi)=\int e^{-i\langle\xi \mid x\rangle / h} u(x) d x$ is the semiclassical Fourier transform and

$$
\widehat{a}(x, z)=\int_{\mathbb{J}^{d}} e^{-i\langle z \mid \xi\rangle / h} a(x, \xi) \frac{d \xi}{(2 \pi h)^{d}}, \quad z \in \mathbb{Z}_{h}^{d},
$$

are the Fourier coefficients of $a(x, \cdot)$. So if $\tilde{f} \in \mathscr{S}\left(\mathbb{R}^{d}\right)$ we get,

$$
\mathrm{Op}_{h}^{W}(a) \tilde{f}(x)=h^{d} \sum_{y \in x+\mathbb{Z}_{h}^{d}} \widehat{a}\left(\frac{x+y}{2}, y-x\right) \tilde{f}(y), \quad x \in \mathbb{R}^{d} .
$$

To apply these remarks to $E^{\prime \prime}(0)$ we consider the Fourier series corresponding to the $V(\cdot, \ell)$,

$$
\widetilde{V}(x, \xi):=\sum_{\substack{\ell \in \mathbb{Z}: \\ 0<|\ell| \leqslant R}} e^{i\langle\xi \mid \ell\rangle} V(x, \ell)=\sum_{\substack{\ell \in \mathbb{Z}: \\ 0<|\ell| \leqslant R}} V(x, \ell) \cos (\langle\xi \mid \ell\rangle), \quad x \in \mathbb{R}^{d}, \xi \in \mathbb{C}_{d} .
$$

(Here the second expression follows from the first since $V$ is even with respect to $\ell$.) As a trigonometric polynomial $\widetilde{V}(x, \cdot)$ is of course entire and $(2 \pi \mathbb{Z})^{d}$-periodic with respect to real $\xi$. By Hypothesis 2.1, $U_{h}, V(\cdot, \ell) \in S_{b}\left(\mathbb{R}^{d}\right)$, and we see that the symbol $U_{h}-\widetilde{V}$ is contained in the class $S_{b}\left(\mathbb{R}^{d} \times \mathbb{R}_{d}\right)$. 
Proposition. 3.1 For all $f \in \ell^{2}\left(\mathbb{Z}_{h}^{d}\right)$ and $\tilde{f} \in C^{\infty}\left(\mathbb{R}^{d}\right)$ with $\left.\tilde{f}\right|_{\mathbb{Z}_{h}^{d}}=f$,

$$
\left(E^{\prime \prime}(0) f\right)(x)=\mathrm{Op}_{h}^{W}\left(U_{h}-\widetilde{V}\right) \tilde{f}(x), \quad x \in \mathbb{Z}_{h}^{d} .
$$

Proof: Since $(\widetilde{V})^{\wedge}(x, z)=h^{-d} V(x, z / h)=h^{-d} V(x,-z / h), 0<|z| \leqslant h R$, and $(\widetilde{V})^{\wedge}(x, z)=$ 0 otherwise, (24) is a special case of (22) with $x \in \mathbb{Z}_{h}^{d}$, provided $\tilde{f} \in \mathscr{S}\left(\mathbb{R}^{d}\right)$. We know, however, that $\mathrm{Op}_{h}^{W}\left(U_{h}-\widetilde{V}\right)$ maps $C^{\infty}\left(\mathbb{R}^{d}\right)$ into itself, because only finitely many Fourier coefficients of $\widetilde{V}$ are non-vanishing. Consequently, (22) is available, for every $\tilde{f} \in C^{\infty}\left(\mathbb{R}^{d}\right)$, in this case.

Proposition 3.1 suggests to study the inverse of $E^{\prime \prime}(0)$ by means of the semiclassical microlocal analysis of the operator $\mathrm{Op}_{h}^{W}\left(U_{h}-\widetilde{V}\right)$. To work out the exponential decay of $\left(E^{\prime \prime}(0)^{-1}\right)_{x y}$, we shall, however, first conjugate the operators in (24) with suitable exponential weights and then construct a parametrix for the conjugated operators. Therefore, we assume that we are given some weight function $\phi \in S_{b}\left(\mathbb{R}^{d}\right)$ in the following and use the symbol $e^{\phi / h}$ also to denote the diagonal multiplication operator on $\ell^{2}\left(\mathbb{Z}_{h}^{d}\right)$, whose action on the canonical orthonormal basis vectors is given by $e^{\phi / h} e_{x}:=e^{\phi(x) / h} e_{x}, x \in \mathbb{Z}_{h}^{d}$. We remark that, eventually, it will be necessary to consider also conjugations with exponential weight functions that depend additionally on a time and a momentum parameter.

In the next lemma we derive a classical asymptoic expansion of the Weyl symbol corresponding to the conjugated operators. As we shall see in the proof this is actually possible using only completely elementary arguments since we are dealing with trigonometric polynomials.

Lemma. 3.2 There is a classical Weyl symbol, $a_{W}^{\phi} \in S_{b}\left(\mathbb{R}^{d} \times \mathbb{R}_{d}\right)$, such that

$$
e^{\phi / h} \mathrm{Op}_{h}^{W}\left(U_{h}-\tilde{V}\right) e^{-\phi / h} \tilde{f}(x)=i \mathrm{Op}_{h}^{W}\left(a_{W}^{\phi}\right) \tilde{f}(x), \quad x \in \mathbb{R}^{d},
$$

and, in particular,

$$
e^{\phi / h} E^{\prime \prime}(0) e^{-\phi / h} f(x)=i \operatorname{Op}_{h}^{W}\left(a_{W}^{\phi}\right) \tilde{f}(x), \quad x \in \mathbb{Z}_{h}^{d},
$$

for all $\tilde{f} \in C^{\infty}\left(\mathbb{R}^{d}\right)$ and $f \in \ell^{2}\left(\mathbb{Z}_{h}^{d}\right)$ such that $\left.\tilde{f}\right|_{\mathbb{Z}_{h}^{d}}=f$. $a_{W}^{\phi}$ is a trigonometric polynomial in $\xi$ and admits a classical asymptotic expansion in $S_{b}\left(\mathbb{R}^{d} \times \mathbb{R}_{d}\right)$,

$$
a_{W}^{\phi}(x, \xi) \asymp \sum_{\nu=0}^{\infty} h^{2 \nu} a_{2 \nu}^{\phi}(x, \xi),
$$

where $a_{0}^{\phi}, a_{2}^{\phi}, \ldots$ are trigonometric polynomials in $\xi$, too. Its principal symbol is given by (recall (15))

$$
a^{\phi}(x, \xi):=a_{0}^{\phi}(x, \xi)=i \tilde{V}\left(x, \xi+i \phi^{\prime}(x)\right)-i U(x), \quad(x, \xi) \in \mathbb{R}^{d} \times \mathbb{R}_{d} .
$$

If $\phi \equiv \phi(t, x, \eta) \in C^{\infty}\left([0, \infty) \times \mathbb{R}^{d} \times \mathbb{R}_{d}\right)$ is such that $\phi+i\langle x \mid \eta\rangle$ defines an element of $S_{b}([0, \infty) \times$ $\left.\mathbb{R}^{d} \times \mathbb{T}^{d}\right)$, then all symbols $a_{W}^{\phi}, a_{0}^{\phi}, a_{2}^{\phi}, \ldots$ can be viewed as elements of $S_{b}\left([0, \infty)_{t} \times \mathbb{R}_{x}^{d} \times \mathbb{R}_{d, \xi} \times \mathbb{T}_{\eta}^{d}\right)$ and the expansion (27) is valid in $S_{b}\left([0, \infty) \times \mathbb{R}^{d} \times \mathbb{R}_{d} \times \mathbb{T}^{d}\right)$.

Proof: First, we consider the multiplication operator $e^{\phi / h} \mathrm{Op}_{h}^{W}\left(U_{h}\right) e^{-\phi / h}=U_{h}$. Using (6), which implies $V(x, \ell)=V(x,-\ell)$, for all $x \in \mathbb{R}^{d}$ and $0<|\ell| \leqslant R$, we write

$$
U_{h}(x)=D_{\theta \theta}^{\prime \prime}(x, 0)+\frac{1}{2} \sum_{0<|\ell| \leqslant R}\left(V\left(x+\frac{h \ell}{2}, \ell\right)+V\left(x-\frac{h \ell}{2}, \ell\right)\right) .
$$

By Taylor's formula, we find, for every $N \in \mathbb{N}$, some $\Psi_{N}(\cdot, \ell ; h) \in S_{b}\left(\mathbb{R}^{d}\right)$ such that

$$
V\left(x+\frac{h \ell}{2}, \ell\right)+V\left(x-\frac{h \ell}{2}, \ell\right)=\sum_{\nu=0}^{N} \frac{2}{(2 \nu) !}\left(\frac{h}{2}\right)^{2 \nu}\langle\ell \mid \nabla\rangle^{2 \nu} V(x, \ell)+h^{2 N+2} \Psi_{N}(x, \ell ; h) .
$$


By (21)-(23) we further have, for all $x \in \mathbb{R}^{d}$,

$$
\begin{aligned}
& e^{\phi / h} \mathrm{Op}_{h}^{W}(\tilde{V}) e^{-\phi / h} \tilde{f}(x) \\
& =h^{d} \sum_{y \in x+\mathbb{Z}_{h}^{d}} \sum_{\substack{\ell \in \mathbb{Z}^{d}: \\
0<|\ell| \leqslant R}} \int_{\mathbb{J}^{d}} e^{i\langle\xi \mid x-y\rangle / h+i\langle\xi \mid \ell\rangle} e^{(\phi(x)-\phi(y)) / h} V\left(\frac{x+y}{2}, \ell\right) \tilde{f}(y) \frac{d \xi}{(2 \pi h)^{d}} .
\end{aligned}
$$

Here the integrals under the double sum are non-zero only if $y-x=h \ell$. If the latter condition is fulfilled we have, however, again by Taylor's formula,

$$
\begin{aligned}
& \frac{1}{h}(\phi(x)-\phi(y))=\frac{1}{h}\left(\phi\left(\frac{x+y}{2}-\frac{h \ell}{2}\right)-\phi\left(\frac{x+y}{2}+\frac{h \ell}{2}\right)\right) \\
& \quad=-\left\langle\ell \mid \nabla \phi\left(\frac{x+y}{2}\right)\right\rangle-\sum_{\nu=1}^{N} \frac{1}{(2 \nu+1) !}\left(\frac{h}{2}\right)^{2 \nu}\langle\ell \mid \nabla\rangle^{2 \nu+1} \phi\left(\frac{x+y}{2}\right)+h^{2 N+2} \Phi_{N}\left(\frac{x+y}{2}, \ell ; h\right),
\end{aligned}
$$

where $\Phi_{N}(\cdot, \ell ; h) \in S_{b}\left(\mathbb{R}^{d}\right)$, for every $N \in \mathbb{N}$. Plugging (30) into (29), writing out the exponential series

$$
\exp \left(-\sum_{\nu=1}^{N} \frac{1}{(2 \nu+1) !}\left(\frac{h}{2}\right)^{2 \nu}\langle\ell \mid \nabla\rangle^{2 \nu+1} \phi\left(\frac{x+y}{2}\right)+h^{2 N+2} \Phi_{N}\left(\frac{x+y}{2}, \ell ; h\right)\right)
$$

and ordering the terms with respect to powers of $h$ we thus get, for every $N \in \mathbb{N}$,

$$
\begin{aligned}
& e^{\phi / h} \operatorname{Op}_{h}^{W}\left(U_{h}-\widetilde{V}\right) e^{-\phi / h} \tilde{f}(x) \\
& \quad=h^{d} \sum_{y \in x+\mathbb{Z}_{h}^{d}} \int_{\mathbb{d}^{d}} e^{i\langle\xi \mid x-y\rangle / h}\left(\sum_{\nu=0}^{N-1} h^{2 \nu} i a_{2 \nu}^{\phi}\left(\frac{x+y}{2}, \xi\right)+h^{2 N} r_{2 N}\left(\frac{x+y}{2}, \xi ; h\right)\right) \tilde{f}(y) \frac{d \xi}{(2 \pi h)^{d}},
\end{aligned}
$$

where $a_{0}^{\phi}, a_{2}^{\phi}, \ldots$ and $r_{2}, r_{4}, \ldots$ are Weyl symbols, which are trigonometric polynomials in $\xi$. For the principal symbol we find

$$
a_{0}^{\phi}(x, \xi)=i \sum_{\substack{\ell \in Z^{d} \\ 0<|\ell| \leqslant R}} V(x, \ell) e^{i\langle\ell \mid \xi\rangle-\left\langle\ell \mid \phi^{\prime}(x)\right\rangle}-i U(x),
$$

which is (28). Applying (21) and (22) to (32) we obtain (25)-(27).

If now $\phi \equiv \phi(t, x, \eta)$ is such that $\phi+i\langle x \mid \eta\rangle$ is $2 \pi$-periodic in $\eta$, we observe first that $\partial_{t} \phi, \phi_{\eta}^{\prime}$, and $\partial_{(t, x, \eta)}^{\alpha} \phi$, for $|\alpha| \geqslant 2$, are again $2 \pi$-periodic functions. For the first derivative with respect to $x$ we get $\phi_{x}^{\prime}(t, x, \eta+e)=\phi_{x}^{\prime}(t, x, \eta)-i e$, for $e \in(2 \pi \mathbb{Z})^{d}$. This shows that all derivatives of $\phi$ appearing in (31) are $2 \pi$-periodic in $\eta$ as well as $e^{-\left\langle\ell \mid \phi_{x}^{\prime}(t, x, \eta)\right\rangle}$, for $\ell \in \mathbb{Z}^{d}, 0<|\ell| \leqslant R$. Therefore, all symbols $a_{0}^{\phi}, a_{2}^{\phi}, \ldots$ and $r_{2}, r_{4}, \ldots$ can be viewed as elements of $S_{b}\left([0, \infty) \times \mathbb{R}^{d} \times \mathbb{R}_{d} \times \mathbb{T}^{d}\right)$, if this is possible for $\phi+i\langle x \mid \eta\rangle$, and it is clear that the last statement holds true.

In order to construct a parametrix for $\mathrm{Op}_{h}^{W}\left(a_{W}^{\phi}\right)$ we will solve a corresponding heat equation by means of a WKB construction. For this purpose we state the following lemma, which is a simple consequence of the method of stationary phase. We suppose again that $\phi$ depends on two additional parameters such that $\phi+i\langle x \mid \eta\rangle \in S_{b}\left([0, \infty) \times \mathbb{R}^{d} \times \mathbb{R}_{d}\right)$ is $2 \pi$-periodic in $\eta$. We further introduce the vector field

$$
Y(t, x, \eta):=\left.\sum_{i=1}^{d} \partial_{\xi^{i}} a^{\phi(t,, \eta)}(x, \xi)\right|_{\xi=0} \partial_{x^{i}}
$$

and set $a_{1}^{\phi} \equiv a_{3}^{\phi} \equiv \cdots \equiv 0$.

Lemma. 3.3 Assume that $b_{\nu} \in S_{b}\left([0, \infty) \times \mathbb{R}^{d} \times \mathbb{T}^{d}\right)$, for $\nu \in \mathbb{N}_{0}$, and suppose that there is some compact subset, $\mathcal{K} \Subset \mathbb{R}^{d}$, such that $\operatorname{supp}\left(b_{\nu}(t, \cdot, \eta)\right) \Subset \mathcal{K}$, for $\nu \in \mathbb{N}_{0}$ and $(t, \eta) \in[0, \infty) \times \mathbb{T}^{d}$. Let 
$b(t, x, \eta ; h)$ be a Borel resummation of the formal series $\sum_{\nu=0}^{\infty} h^{\nu} b_{\nu}(t, x, \eta)$. Then we find symbols $\widetilde{r}_{N} \in S_{b}\left([0, \infty) \times \mathbb{R}^{d} \times \mathbb{T}^{d}\right), N \in \mathbb{N}$, with

$$
\operatorname{supp}\left(\widetilde{r}_{N}(t, \cdot, \eta)\right) \subset \mathcal{K}+\{|x| \leqslant R\} \quad(t, \eta) \in[0, \infty) \times \mathbb{T}^{d},
$$

such that, for all $(t, x, \eta) \in[0, \infty) \times \mathbb{R}^{d} \times \mathbb{T}^{d}$ and sufficiently small $h>0$,

$$
\begin{aligned}
& \left(i \mathrm{Op}_{h}^{W}\left(a_{W}^{\phi}\right) b\right)(t, x, \eta) \\
& =\quad i a^{\phi(t,,, \eta)}(x, 0) b(t, x, \eta ; h)+h\left(\left(Y+\frac{1}{2} \operatorname{div} Y\right) b_{0}\right)(t, x, \eta) \\
& \quad+\sum_{\nu=2}^{N-1} h^{\nu}\left(\left(\left(Y+\frac{1}{2} \operatorname{div} Y\right) b_{\nu-1}\right)(t, x, \eta)+\sum_{\varkappa=0}^{\nu-2}\left(P_{\nu-\varkappa} b_{\varkappa}\right)(t, x, \eta)\right) \\
& \quad+h^{N} \widetilde{r}_{N}(t, x, \eta ; h),
\end{aligned}
$$

where, for $\tilde{f} \in C^{\infty}\left(\mathbb{R}^{d}\right)$,

$$
\left(P_{\nu} \tilde{f}\right)(t, x, \eta)=\left.i \sum_{\varkappa=0}^{\nu} \frac{1}{i^{\varkappa} \varkappa !}\left(\sum_{i=1}^{d} \partial_{y^{i}} \partial_{\xi_{i}}\right)^{\varkappa}\left(a_{\nu-\varkappa}^{\phi(t, \cdot, \eta)}\left(\frac{x+y}{2}, \xi\right) \tilde{f}(y)\right)\right|_{\xi=0, y=x} .
$$

\section{Construction of a weight function}

In order to study the exponential decay of the Green kernel of $E^{\prime \prime}(0)$ we distinguish two points $x^{\star}$ and $y^{\star}$ and design a suitable weight function, $\varphi$, which models the exponential decay of $\left(E^{\prime \prime}(0)^{-1}\right)_{x^{\star} y^{\star}}$. Here we can choose any pair of points $x^{\star}, y^{\star}$ satisfying a certain condition introduced below. An appropriate weight function will essentially be given as a solution of the Hamilton-Jacobi equation

$$
T\left(x, \varphi^{\prime}(x)\right)-U(x)=0,
$$

where $T(x, p):=\widetilde{V}(x, i p)$. For our purposes it is actually sufficient to solve (34) only in a small neighbourhood of a certain Finsler geodesic from $y^{\star}$ to $x^{\star}$. The solution $\varphi$ will then be extended in such a way that, for all $x$ outside that neighbourhood, it holds $T\left(x, \varphi^{\prime}(x)\right)-U(x)<0$. The construction of $\varphi$ is presented in Subsection 4.1 below. In Subsection 4.2 we collect various properties of the principal symbol $a^{\varphi}$ defined by (28) with $\phi=\varphi$, which play an important role in the sequel.

\subsection{The construction of $\varphi$}

There is no reason to restrict ourselves to the special Hamilton function $T-U$ appearing in (34) for the purpose of this subsection. In Subsection 4.2 we will observe that $T-U$ is an element of the set of all Hamilton functions, $H$, that satisfy

Hypothesis. 4.1 It holds $H \in C^{\infty}\left(T^{*} \mathbb{R}^{d}, \mathbb{R}\right)$. For all $x \in \mathbb{R}^{d}$, the function $H(x, \cdot): T_{x}^{*} \mathbb{R}^{d} \rightarrow \mathbb{R}$ is strictly convex, even, and $H(x, 0)<0$.

We thus seek for solutions of the Hamilton-Jacobi equation $H\left(x, \varphi^{\prime}(x)\right)=0$, where $H$ satisfies Hypothesis 4.1. The appropriate solution will essentially be given by the Finsler distance function to some prescribed point on the prolongation of the geodesic from $y^{\star}$ to $x^{\star}$. Before we turn to the construction of $\varphi$ we recall some required notions and facts from Finsler geometry. In Section 8 we shall also make use of them. All the standard results collected below can be found, e.g., in [5, 6, 11.

We have already introduced the polar bodies

$$
\mathbb{k}_{x}^{*}:=\left\{p \in \mathbb{R}_{d}=T_{x}^{*} \mathbb{R}^{d}: H(p, x) \leqslant 0\right\}, \quad x \in \mathbb{R}^{d} .
$$


For every $x \in \mathbb{R}^{d}, \mathbb{R}_{x}^{*}$ is strictly convex and symmetric about the origin. Its boundary,

$$
\mathbb{F}_{x}:=\left\{p \in T_{x}^{*} \mathbb{R}^{d}: H(p, x)=0\right\},
$$

which is called the figuratrix at $x$, is a smooth submanifold. We further set $\mathbb{f}:=H^{-1}(\{0\})$. We also recall that the support function, $F(x, \cdot): \mathbb{R}^{d}=T_{x} \mathbb{R}^{d} \rightarrow[0, \infty)$, of $\mathbb{k}_{x}^{*}$ is given by

$$
F(x, v):=\sup \left\{\langle p \mid v\rangle: p \in \mathbb{R}_{x}^{*}\right\}, \quad v \in \mathbb{R}^{d} .
$$

The set

$$
\mathbb{k}_{x}:=\left\{v \in \mathbb{R}^{d}=T_{x} \mathbb{R}^{d}: F(x, v) \leqslant 1\right\}
$$

is again strictly convex and symmetric about the origin, and its smooth boundary,

$$
\stackrel{\circ}{\triangleright}_{x}:=\left\{v \in T_{x} \mathbb{R}^{d}: F(x, v)=1\right\},
$$

is called the indicatrix at $x \in \mathbb{R}^{d}$. For $v \neq 0$, the value of the support function is given by $F(x, v)=\langle p(x, \stackrel{v}{v}) \mid v\rangle$, where $p(x, \stackrel{\circ}{v})$ is the unique point on $\mathbb{E}_{x}$ at which the exterior normal field on $\mathbb{E}_{x}$, i.e. the normalized gradient of $H$ with respect to $p$, equals $\stackrel{i}{v}:=v /|v|$. The function $F$ is continuous on $T \mathbb{R}^{d}$ and smooth on $\dot{T} \mathbb{R}^{d}$. (Here and in the following we write $\dot{T} \mathbb{R}^{d}$ for the tangent bundle with the zero section deleted and use a similar notation for the cotangent bundle.) Moreover, $F(x, \cdot)$ is absolutely homogenous of degree one, i.e. $F(x, \theta v)=|\theta| F(x, v)$, for all $(x, v) \in T \mathbb{R}^{d}$ and $\theta \in \mathbb{R}$. Euler's theorem thus implies

$$
F_{v}^{\prime}(x, v)=p(x, \stackrel{o}{v}) \quad \text { and } \quad F_{v v}^{\prime \prime}(x, v) v=0, \quad(x, v) \in \dot{T} \mathbb{R}^{d} .
$$

The restriction of $F_{v v}^{\prime \prime}(x, v)$ to the orthogonal complement of $v$ is strictly positive. The matrix $G$ with entries

$$
G_{i j}:=\left(\frac{1}{2} F^{2}\right)_{v^{i} v^{j}}^{\prime \prime}=F F_{v^{i} v^{j}}^{\prime \prime}+F_{v^{i}}^{\prime} F_{v^{j}}^{\prime}, \quad 1 \leqslant i, j \leqslant d,
$$

is therefore positive definite at every point of $\dot{T} \mathbb{R}^{d}$. Altogether $F$ enjoys all properties required for a Finsler structure on $T \mathbb{R}^{d}$; see, e.g., 5 . In the calculus of variations $F$ is called an elliptic parametric Lagrangean and $H$ is an associated Hamiltonian in the sense of Carathéodory, as $H_{p}^{\prime} \neq 0$ on $\dot{T}^{*} \mathbb{R}^{d}$ and $H\left(x, F_{v}^{\prime}(x, v)\right)=0$, for $(x, v) \in \dot{T} \mathbb{R}^{d}$; see, e.g., [6]. We also introduce

\section{Hypothesis. 4.2}

$$
\inf \left\{F(x, \stackrel{\imath}{v}) \mid x \in \mathbb{R}^{d}, \stackrel{\circ}{v} \in S^{d-1}\right\}>0 .
$$

Since $F$ is absolutely homogenous of degree one in $v$, the map $d_{F}: \mathbb{R}^{d} \times \mathbb{R}^{d} \rightarrow[0, \infty)$, given by

$$
d_{F}(x, y):=\inf _{q} \mathscr{A}(q), \quad \mathscr{A}(q):=\int F(q, \dot{q}),
$$

defines a metric on $\mathbb{R}^{d}$. Here the infimum is taken over all piecewise smooth curves in $\mathbb{R}^{d}$ from $y$ to $x$. (One could equally well take the minimum over Lipschitz continuous curves defined on the unit interval, which would give the same results.) In [11, §5.3] it is shown that

$$
\widetilde{\varphi}(x):=d_{F}(x, y), \quad x \in \mathbb{R}^{d},
$$

is the (unique) viscosity solution of

$$
H\left(x, \widetilde{\varphi}^{\prime}(x)\right)=0, \text { for } x \in \mathbb{R}^{d} \backslash\{y\}, \quad \widetilde{\varphi}(y)=0 .
$$

In particular, this means that $\widetilde{\varphi}$ is continuous, differentiable on $\mathbb{R}^{d} \backslash(\{y\} \cup \Sigma)$, where $\Sigma$ is some closed set of finite $(d-1)$-dimensional Hausdorff measure [10], and satisfies $H\left(x, \widetilde{\varphi}^{\prime}(x)\right)=0$, for $x \in \mathbb{R}^{d} \backslash(\{y\} \cup \Sigma)$. Moreover, it is known that, for any function $u: \mathbb{R}^{d} \rightarrow \mathbb{R}$ with $u(y)=0$, which is locally Lipschitz continuous and satisfies $H\left(x, u^{\prime}(x)\right) \leqslant 0$ almost everywhere, it holds $u \leqslant \widetilde{\varphi}$. In 
Section 5 it will become clear that the latter property shows that $\widetilde{\varphi}$ is an optimal weight function for our purposes. Since it is not smooth we have, however, to modify it suitably. To this end we recall some further notions from Finsler geometry in the following.

Extremals of the functional $\mathscr{A}$ are called geodesics. It is well-known that under Hypothesis 4.2 the infimum in (37) is actually attained and that each geodesic is smooth. The homogenity of $F$ implies that $\mathscr{A}$ is invariant under reparametrizations. In particular, every reparametrization of a geodesic is again a geodesic. Moreover, we have the following well-known lemma relating the geodesics to trajectories of the Hamiltonian vector field

$$
X_{H}:=\left(\begin{array}{c}
\nabla_{p} H \\
-\nabla_{x} H
\end{array}\right) .
$$

Its proof can be found, e.g., in [6, pp. 197].

Lemma. 4.3 Any geodesic defined by means of $F$ can be reparametrized in such a way, that we obtain the projection onto $\mathbb{R}^{d}$ of a Hamiltonian trajectory defined by means of $H$ and running in $\mathbb{E} \subset T^{*} \mathbb{R}^{d}$. Conversely, any projection of a Hamiltonian trajectory running in $\mathbb{E}$ is a geodesic.

Next, we recall that under Hypothesis 4.2 the exponential map determined by the Finsler structure $F$ is defined on $T \mathbb{R}^{d}$ by $\exp _{x}(v):=q_{x, v}(1)$, for $(x, v) \in T \mathbb{R}^{d}, v \neq 0$, and $\exp _{x}(0):=x$, for $x \in \mathbb{R}^{d}$. Here $q_{x, v}$ is the unique geodesic which passes through $x$ at time $t=0$ with velocity $v$. (It holds $q_{x, v}(\theta t)=q_{x, \theta v}(t), \theta>0$.) If $v \in \stackrel{\circ}{x}_{x}$, then $t \mapsto q_{x, v}(t)=\exp _{x}(t v)$ is the unit speed geodesic passing through $x$ at $t=0$ in the direction of $v$, that is, $F\left(q_{x, v}(t), \dot{q}_{x, v}(t)\right)=1$, for all $t \in \mathbb{R}$. We remark that the projections of the Hamiltonian trajectories onto $\mathbb{R}^{d}$ might have different velocities.

We assume that $x, y, v \in \mathbb{R}^{d}$, and $x=\exp _{y}(v)$ in the following. If the derivative of $\exp _{y}$ : $T_{y} \mathbb{R}^{d} \rightarrow \mathbb{R}^{d}$ is singular at $v$, then $x$ is called a conjugate point for $y$. If $x$ is the first conjugate point for $y$ along the geodesic $t \mapsto q(t):=\exp _{y}(t v)$, then we know that, for all $\tau \in(0,1), q \uparrow_{[0, \tau]}$ is, again up to reparametrization, the unique minimizing geodesic among all geodesics from $y$ to $x$ that run in some small neighbourhood of $q([0, \tau])$. It does not have, however, to be globally minimizing. For $\tau>1, q \uparrow_{[0, \tau]}$ will definitely lose its unique minimizing property even among nearby geodesics. The cut point of $y$ along $q$ is by definition $q(r)$, where $r$ is the supremum of all those $\tau>0$ such that $q \uparrow_{[0, \tau]}$ is globally minimizing. The cut point of $y$ along $q$ always appears before or at the first conjugate point. Notice that, if $x$ is simultanously the cut and first conjugate point for $y$ along $q$, then $q \uparrow_{[0,1]}$ might still be globally minimizing. The unique minimizing property of $q$ will, however, definitely fail at the cut point, if the latter occurs strictly before the first conjugate point.

We can always find some open set, $\mathscr{W} \subset T_{y} \mathbb{R}^{d}$, which is star-shaped with respect to zero, such that $\exp _{y}\left\lceil\mathscr{W} \in C^{1}(\mathscr{W}) \cap C^{\infty}(\mathscr{W} \backslash\{0\})\right.$ is bijective from $\mathscr{W}$ onto $\mathscr{U}:=\exp _{y}(\mathscr{W})$, and it is well-known that

$$
\widetilde{\varphi}(x)=d_{F}(x, y)=F\left(y,\left(\exp _{y}\lceil\mathscr{W})^{-1}(x)\right), \quad x \in \mathscr{U}\right.
$$

see, e.g., [6, Chapter 8, §3.3]. Moreover, we have

$$
\widetilde{\varphi}^{\prime}(x)=F_{v}^{\prime}(y, \dot{\gamma}(\tau))=\xi(\tau), \quad x \in \mathscr{U} \backslash\{y\},
$$

where $t \mapsto(\gamma(t), \xi(t)) \in \mathbb{E}$ is the Hamiltonian trajectory corresponding to the minimizing geodesic from $y$ to $x$ such that $\gamma(0)=y$ and $\gamma(\tau)=x$, for some $\tau>0$.

In the following we fix two distinguished points $x^{\star}, y^{\star} \in \mathbb{R}^{d}$, and assume

Hypothesis. $4.4 x^{\star} \neq y^{\star}$. Up to reparametrization, there is a unique minimizing geodesic from $y^{\star}$ to $x^{\star} . x^{\star}$ and $y^{\star}$ are not conjugate to each other.

We recall that Hypothesis 4.4 is always fulfilled provided $x^{\star}$ and $y^{\star}$ are sufficiently close to each other. Since $F$ is absolutely homogenous there exists of course, up to reparametrization, again only one minimizing geodesic from $x^{\star}$ to $y^{\star}$ if Hypothesis 4.4 is satisfied. Moreover, we can prolong the geodesic from $y^{\star}$ to $x^{\star}$ or from $x^{\star}$ to $y^{\star}$ a little bit such that it is still minimizing. 
Proposition. 4.5 Assume that $H \in C^{\infty}\left(T^{*} \mathbb{R}^{d}, \mathbb{R}\right)$ fulfills Hypotheses 4.1834 .2 and that $x^{\star}, y^{\star} \in$ $\mathbb{R}^{d}$ fulfill Hypothesis 4.4 . Then there exist a point, $y_{0}$, on the prolongation of the geodesic from $x^{\star}$ to $y^{\star}$, a compact neighbourhood, $K_{0}$, of the geodesic segment from $y^{\star}$ to $x^{\star}$, some open set, $\mathscr{W} \subset T_{y_{0}} \mathbb{R}^{d}$, which is star-shaped with respect to zero, and some function, $\varphi \in C_{b}^{\infty}\left(\mathbb{R}^{d}, \mathbb{R}\right)$, such that the following holds true:

(i) For all $x \in \mathbb{R}^{d}$,

$$
\begin{aligned}
& H\left(x, \varphi^{\prime}(x)\right) \leqslant 0 \\
& H\left(x, \varphi^{\prime}(x)\right)=0 \Leftrightarrow x \in K_{0} .
\end{aligned}
$$

(ii) $\varphi(x)-\varphi\left(y^{\star}\right)=d_{F}\left(x, y^{\star}\right)$, for all $x$ on the geodesic segment from $y^{\star}$ to $x^{\star}$.

(iii) $\exp _{y_{0}}\left\lceil\mathscr{W} \in C^{1}(\mathscr{W}) \cap C^{\infty}(\mathscr{W} \backslash\{0\})\right.$ is injective on $\mathscr{W}$ and

$$
K_{0} \subset \exp _{y_{0}}(\mathscr{W}) \backslash\left\{y_{0}\right\} .
$$

(iv) For every $x \in K_{0}$, there is a unique pair $\left(\tau, p_{0}\right) \in(0, \infty) \times \mathbb{E}_{y_{0}}$ such that the projection of $[0, \tau] \ni t \mapsto \exp \left(t X_{H}\right)\left(y_{0}, p_{0}\right)$ onto $\mathbb{R}^{d}$ is a minimizing geodesic from $y_{0}$ to $x$. We have

$$
\exp \left(\tau X_{H}\right)\left(y_{0}, p_{0}\right)=\left(x, \varphi^{\prime}(x)\right) .
$$

Proof: Let $I$ be an open interval and $q: I \rightarrow \mathbb{R}^{d}$ be a geodesic with $q\left(t_{1}\right)=y^{\star}$ and $q\left(t_{2}\right)=x^{\star}$, for some $t_{1}, t_{2} \in I, t_{1}<t_{2}$. We can find some $t_{0} \in I, t_{0}<t_{1}$, such that $q\left\lceil_{\left[t_{0}, t_{2}\right]}\right.$ is still, up to reparametrizations, the unique geodesic segment from $y_{0}:=q\left(t_{0}\right)$ to $x^{\star}$. We can moreover find some open set $\mathscr{W} \subset T_{y_{0}} \mathbb{R}^{d}$, star-shaped with respect to zero, such that the restriction of $\exp _{y_{0}}$ to $\mathscr{W}$ is bijective from $\mathscr{W}$ onto $\mathscr{U}:=\exp _{y_{0}}(\mathscr{W})$, where $q\left(\left[t_{0}, t_{2}\right]\right) \subset \mathscr{U}$. We have $\exp _{y_{0}} \in C^{1}(\mathscr{W}) \cap$ $C^{\infty}(\mathscr{W} \backslash\{0\})$. Defining $\widetilde{\varphi}$ by (38) with $y=y_{0}$ we know that $\widetilde{\varphi}(x)=F\left(y_{0},\left(\exp _{y_{0}}\lceil\mathscr{W})^{-1}(x)\right)\right.$, $x \in \mathscr{U}$, solves $H\left(x, \varphi^{\prime}(x)\right)=0$ on $\mathscr{U} \backslash\left\{y_{0}\right\}$.

Next, we pick some open set, $\mathscr{U}_{\star}$, and two compact sets, $K_{\star}$ and $K$, with

$$
q\left(\left[t_{0}, t_{2}\right]\right) \Subset \stackrel{\circ}{K}_{\star} \subset K_{\star} \Subset \stackrel{\circ}{K} \subset K \Subset \mathscr{U}_{\star} \subset \overline{\mathscr{U}}_{\star} \Subset \mathscr{U} .
$$

We suppose that the boundaries $\partial K_{\star}$ and $\partial K$ are smooth submanifolds. Furthermore, we can assume that $\exp _{y_{0}}^{-1}\left(K_{\star}\right), \exp _{y_{0}}^{-1}(K)$, and $\exp _{y_{0}}^{-1}\left(\mathscr{U}_{\star}\right)$ are star-shaped with respect to zero. We pick some $\widetilde{H} \in C_{b}^{\infty}\left(\mathbb{R}^{d}, \mathbb{R}\right)$ with $\widetilde{H} \equiv 0$ on $K_{\star}$, and $0<\widetilde{H}(x) \leqslant-H(x, 0)$, for $x \in \mathbb{R}^{d} \backslash K_{\star}$, and set

$$
H_{\varepsilon}:=H+\varepsilon \widetilde{H}, \quad \varepsilon \in(0,1] .
$$

For every $\varepsilon \in(0,1]$, we let $\varphi_{\varepsilon}$ denote the function constructed exactly in the same way as $\widetilde{\varphi}$ using the Hamiltonian $H_{\varepsilon}$ instead. We notice that $\varphi_{\varepsilon} \equiv \widetilde{\varphi}$ on $K_{\star}$, since the Finsler structures and, hence, the geodesics emanating from $y_{0}$ corresponding to $H_{\varepsilon}$ and $H$ are identical on $K_{\star}$.

In the following we show that, for all sufficiently small $\varepsilon \in(0,1], \varphi_{\varepsilon}$ is, apart from $y_{0}$, smooth at every point in a neighbourhood of $K$. We know that all geodesics of the Hamiltonian $H$ contained in $\mathscr{U}$ and emanating from $y_{0}$ form a central field about $y_{0}$. No conjugate or cut points of $y_{0}$ are present in $\mathscr{U}$. First we claim

Lemma. 4.6 There is some $\varepsilon_{0} \in(0,1]$ such that, for all $\varepsilon \in\left(0, \varepsilon_{0}\right]$, every geodesic of the Hamiltonian $H_{\varepsilon}$ emanating from $y_{0}$ leaves $K_{\star}$ and touches a point of $\mathbb{R}^{d} \backslash \mathscr{U}_{\star}$ before it possibly enters $K_{\star}$ again.

Proof: Let $v \in{\stackrel{\circ}{y_{0}}}_{0}$, and let $\tau>0$ be the first exit time of $K_{\star}$ for the geodesic $t \mapsto \exp _{y_{0}}(t v)$ defined by $H$. Then the geodesics $t \mapsto \exp _{y_{0}}(t v)$ and $t \mapsto \exp _{y_{0}}^{\varepsilon}(t v)$ are identical on $[0, \tau]$, for $\varepsilon \in(0,1]$. Here we designate the exponential map defined by $H_{\varepsilon}$ by a superscript $\varepsilon$. We also know that $t \mapsto \exp _{y_{0}}(t v)$ touches a point in $\mathbb{R}^{d} \backslash \mathscr{U}$, say, at time $\bar{t}>0$, before it possibly enters 
$K_{\star}$ again. There is an open neighbourhood, $\mathscr{I}_{v} \subset{\stackrel{\circ}{0} y_{0}}_{0}$ of $v$ in the indicatrix at $y_{0}$ such that $\exp _{y_{0}}(\bar{t} w) \in \mathbb{R}^{d} \backslash \mathscr{U}$, for all $w \in \mathscr{I}_{v}$. Since all geodesics depend smoothly on the parameter $\varepsilon$ and on $w \in \mathscr{I}_{v}$, we find some $\varepsilon_{v} \in(0,1]$ such that $\exp _{y_{0}}^{\varepsilon}(\bar{t} w) \in \mathbb{R}^{d} \backslash \mathscr{U}_{\star}$, for all $w \in \mathscr{I}_{v}$ and $\varepsilon \in\left(0, \varepsilon_{v}\right]$. The claim now follows from the compactness of ${\stackrel{\circ}{0} y_{0}}$. For the open cover $\bigcup_{v \in \in_{y_{0}}^{\circ}} \mathscr{I}_{v}$ contains a finite subcover $\bigcup_{\iota=1}^{\nu} \mathscr{I}_{v_{\iota}}$ and we can set $\varepsilon_{0}:=\min \left\{\varepsilon_{v_{1}}, \ldots, \varepsilon_{v_{\nu}}\right\}$.

Lemma. 4.7 There is some $\varepsilon_{1}>0$ such that, for all $\varepsilon \in\left(0, \varepsilon_{1}\right]$, there is no conjugate or cut point for $y_{0}$ contained in $K$, if the geodesics are defined using the Hamiltonian $H_{\varepsilon}$.

ProOF: The assertion about the conjugate points is clear since they are characterized by singularities of the exponential map, which depends smoothly on $\varepsilon$. Concerning the cut points we prove a slightly stronger statement, namely that there are $\varepsilon_{1} \in(0,1]$ and $c>0$ such that, for all $\varepsilon \in\left(0, \varepsilon_{1}\right]$, and every pair of unit speed geodesics, $q_{1}^{\varepsilon}$ and $q_{2}^{\varepsilon}, q_{1}^{\varepsilon} \neq q_{2}^{\varepsilon}$, defined by $H_{\varepsilon}$ and emanating from $y_{0}$, we have

$$
\left(\exists t, \tilde{t}>0: q_{1}^{\varepsilon}([0, t]) \subset K \wedge q_{1}^{\varepsilon}(t)=q_{2}^{\varepsilon}(\tilde{t})\right) \Longrightarrow \tilde{t} \geqslant t+c .
$$

Note that, for unit speed geodesics, the time parameter equals the Finsler arc length. We argue by contradiction and suppose that there are sequences, $\left(\varepsilon_{n}\right),\left(c_{n}\right),\left(t_{n}\right),\left(\tilde{t}_{n}\right),\left(v_{n}\right),\left(\tilde{v}_{n}\right)$, such that $\varepsilon_{n} \searrow 0, c_{n} \searrow 0$, as $n \rightarrow \infty$, and $t_{n}, \tilde{t}_{n}>0, v_{n}, \tilde{v}_{n} \in{\stackrel{\circ}{x^{\star}}}^{\star}, v_{n} \neq \tilde{v}_{n}$, for $n \in \mathbb{N}$, which satisfy $\tilde{t}_{n}<t_{n}+c_{n}$,

$$
\exp _{y_{0}}^{\varepsilon_{n}}\left(t_{n} v_{n}\right)=\exp _{y_{0}}^{\varepsilon_{n}}\left(\tilde{t}_{n} \tilde{v}_{n}\right), \quad \text { and } \quad \exp _{y_{0}}^{\varepsilon_{n}}\left(s v_{n}\right) \in K, s \in\left[0, t_{n}\right],
$$

for $n \in \mathbb{N}$. By definition of $K_{\star}$ we know that the unit speed geodesic with initial velocity $\tilde{v}_{n}$ does not stay inside $K_{\star}$, for all $t \in\left[0, \tilde{t}_{n}\right]$. By Lemma 4.6 we thus find $\tau_{n} \in\left(0, \tilde{t}_{n}\right)$ such that $\exp _{y_{0}}^{\varepsilon_{n}}\left(\tau_{n} \tilde{v}_{n}\right) \in \mathscr{U}_{\star}^{c}$, for $n \in \mathbb{N}$. By compactness and by a choice of suitable subsequences we can assume that the sequences $\left(t_{n}\right),\left(\tilde{t}_{n}\right),\left(v_{n}\right),\left(\tilde{v}_{n}\right)$, and $\left(\tau_{n}\right)$, have a limit, as $n$ tends to infinity, which we denote by $t, \tilde{t}, v, \tilde{v}$, and $\tau$, respectively. Then it holds $\tilde{t} \leqslant t$, $\exp _{y_{0}}(\tau \tilde{v}) \in \mathscr{U}_{\star}^{c}$,

$$
\exp _{y_{0}}(t v)=\exp _{y_{0}}(\tilde{t} \tilde{v}) \in K, \quad \text { and } \quad \exp _{y_{0}}(s v) \in K, s \in[0, t]
$$

It also follows that $v \neq \tilde{v}$ and we get a contradiction, because $[0, t] \ni s \mapsto \exp _{y_{0}}(s v)$ is the unique minimizing geodesic for $H$ from $y_{0}$ to $\exp _{y_{0}}(t v)$.

Returning to the proof of Proposition 4.5 we notice that Lemma 4.7 implies that, for all $\varepsilon \in\left(0, \varepsilon_{1}\right]$, $\varphi_{\varepsilon}$ is smooth in a (in general $\varepsilon$-dependent) neighbourhood of $K \backslash\left\{y_{0}\right\}$, since the set where $\varphi_{\varepsilon}$ is not smooth is closed.

Next, we pick some compact set, $K^{\prime}$, satisfying $K_{\star} \Subset \stackrel{\circ}{K^{\prime}} \subset K^{\prime} \Subset \stackrel{\circ}{K}$, and some cut-off function $\chi \in C^{\infty}\left(\mathbb{R}^{d},[0,1]\right)$ such that $\chi \equiv 1$ on $K^{\prime}$ and $\chi \equiv 0$ on $K^{c}$. We note that there is some $C>0$ such that $H_{\varepsilon}-H \geqslant C \varepsilon$ on $\operatorname{supp}\left(\chi^{\prime}\right)$. We further pick a family, $\left\{j_{\delta}\right\}_{\delta>0}$, of Friedrichs mollifiers such that $\operatorname{supp}\left(j_{\delta}\right) \Subset\{|x| \leqslant \delta\}$ and observe that

$$
\left(\chi \varphi_{\varepsilon}+(1-\chi) j_{\delta} * \varphi_{\varepsilon}\right)^{\prime}=\chi \varphi_{\varepsilon}^{\prime}+(1-\chi) j_{\delta} * \varphi_{\varepsilon}^{\prime}+\left(\varphi_{\varepsilon}-j_{\delta} * \varphi_{\varepsilon}\right) \chi^{\prime}
$$

holds on $\mathbb{R}^{d} \backslash\left\{y_{0}\right\}$. Here we note that the expression $\left(j_{\delta} * \varphi_{\varepsilon}^{\prime}\right)\left(\underset{\widetilde{H}}{)}\right.$ makes sense, also for $x \in K^{c}$, since $\varphi_{\varepsilon}$ is differentiable almost everywhere. Since the function $\widetilde{H}$ appearing in the definition (40) of $H_{\varepsilon}$ depends only on $x$, the set

$$
\mathbb{k}_{x}^{\varepsilon}:=\left\{p \in \mathbb{R}_{d}: H_{\varepsilon}(x, p) \leqslant 0\right\}
$$

is still convex, and by the properties of $\widetilde{H}$ it holds $\mathbb{k}_{x}^{\varepsilon} \subset \mathbb{k}_{x}$, for all $x \in \mathbb{R}^{d}$. This inclusion is proper if $x \in K_{\star}^{c}$. Moreover, we have $H_{\varepsilon}\left(x, \varphi_{\varepsilon}^{\prime}(x)\right)=0$, that is, $\varphi_{\varepsilon}^{\prime}(x) \in \mathbb{k}_{x}^{\varepsilon}$, for almost every $x \in \mathbb{R}^{d}$. Since $j_{\delta}$ is a probability density and $\mathbb{k}_{x}^{\varepsilon}$ is convex, it holds $\left(j_{\delta} * \varphi_{\varepsilon}^{\prime}\right)(x) \in \mathbb{k}_{x}^{\varepsilon}$, for all $x \in \mathbb{R}^{d}$. Consequently, for every $x \in \mathbb{R}^{d} \backslash\left\{y_{0}\right\}$ the convex combination $\chi(x) \varphi_{\varepsilon}^{\prime}(x)+(1-\chi(x))\left(j_{\delta} * \varphi_{\varepsilon}^{\prime}\right)(x)$ is 
contained in $\mathbb{k}_{x}^{\varepsilon}$, too. On the support of $\chi^{\prime}$ the set $\mathbb{R}_{x}^{\varepsilon}$ and the indicatrix ${ }_{i}$ have a strictly positive distance which is uniformly bounded from below by some contant $\widetilde{C}(\varepsilon)>0$. We also know that the term $\left(\varphi_{\varepsilon}-j_{\delta} * \varphi_{\varepsilon}\right)$ converges to zero uniformly on the compact set $\operatorname{supp}\left(\chi^{\prime}\right)$, as $\delta$ tends to zero. Choosing $\delta>0$ small enough, we can therefore ensure that the right side of (41) is contained in $\mathbb{k}_{x}$, for all $x \in \mathbb{R}^{d} \backslash\left\{y_{0}\right\}$.

The function $\chi \varphi_{\varepsilon}+(1-\chi) j_{\delta(\varepsilon)} * \varphi_{\varepsilon}$ is smooth everywhere except at $y_{0}$. To obtain a smooth function we pick some $\Theta \in C^{\infty}(\mathbb{R},[0, \infty))$ such that $0 \leqslant \Theta^{\prime} \leqslant 1, \Theta=$ const $>0$ in a neighbourhood of zero, $\Theta(t)=t$, for all $d_{F}\left(y_{0}, y^{\star}\right) / 2<t<2 d_{F}\left(y_{0}, x^{\star}\right)$, and $\Theta=$ const, on $\left[3 d_{F}\left(y_{0}, x^{\star}\right), \infty\right)$. Finally, we choose some $\varepsilon \in\left(0, \varepsilon_{1}\right]$, and some sufficiently small $\delta(\varepsilon)>0$, and set

$$
\varphi:=\Theta \circ\left(\chi \varphi_{\varepsilon}+(1-\chi) j_{\delta(\varepsilon)} * \varphi_{\varepsilon}\right) .
$$

We observe that we still have $\varphi^{\prime}(x) \in \mathbb{k}_{x}$, for $x \in \mathbb{R}^{d}$, since $0 \leqslant \Theta^{\prime} \leqslant 1$. To complete the proof we note that, by the minimizing property of the geodesic from $y_{0}$ to $x^{\star}$,

$$
\varphi(x)-\varphi\left(y^{\star}\right)=d_{F}\left(x, y_{0}\right)-d_{F}\left(y^{\star}, y_{0}\right)=d_{F}\left(x, y^{\star}\right),
$$

for all $x$ on the unique geodesic from $y^{\star}$ to $x^{\star}$. Finally, (iii) is fulfilled by construction and (iv) follows from (39).

\subsection{The principal symbol $a^{\varphi}$}

We turn to the discussion of the special Hamilton function $H:=T-U$, where

$$
T(x, p)=\widetilde{V}(x, i p)=\sum_{\substack{\ell \in \mathbb{Z}^{d:} \\ 0<|\ell| \leqslant R}} V(x, \ell) \cosh (\langle\ell \mid p\rangle), \quad x \in \mathbb{R}^{d}, p \in \mathbb{R}_{d},
$$

and $U$ is given by (15). First, we observe as in [20, §4] that, for every $x \in \mathbb{R}^{d}$, the function $T(x, \cdot): \mathbb{R}_{d} \rightarrow(0, \infty)$ is strictly convex and even. Moreover, $0<T(x, 0) \leqslant U(x)-\varrho$, for some $x$ independent $\varrho>0$, provided $J>0$ is sufficiently small. In other words, $H$ satisfies Hypothesis 4.1 . Using the uniform bounds on $U(x)$ and $V(\ell, x)$ of Hypothesis 2.1] it is not difficult to see that Hypothesis 4.2 is fulfilled by $H$, too. So, Proposition 4.5 is applicable, for each pair of points $x^{\star}, y^{\star}$ satisfying Hypothesis 4.4 .

Since we want to use the results of Section 3 with $\phi=\varphi$, we collect some important properties of the principal symbol

$$
a^{\varphi}(x, \xi)=i \widetilde{V}\left(x, \xi+i \varphi^{\prime}(x)\right)-i U(x), \quad(x, \xi) \in \mathbb{R}^{d} \times \mathbb{R}_{d},
$$

in the next lemma. In its statement and henceforth we use the short-hand notation $\left(a^{\varphi}\right)_{\xi x}^{\prime \prime}:=$ $d_{x} \nabla_{\xi} a^{\varphi}$ etc. The Hamiltonian vector field of $\Re a^{\varphi}$ is denoted by $X_{\Re a^{\varphi}}:=\left(\begin{array}{c}\nabla_{\xi} \Re a^{\varphi} \\ -\nabla_{x} \Re a^{\varphi}\end{array}\right)$.

Lemma. 4.8 Fix two distinguished points $x^{\star}, y^{\star} \in \mathbb{R}^{d}$ fulfilling Hypothesis 4.4 and let $\varphi$ and $K_{0}$ be as in Proposition 4.5. Then the symbol $a^{\varphi} \in S_{b}\left(\mathbb{R}^{d} \times \mathbb{R}_{d}\right)$ has the following properties:

(i) For $(x, \xi) \in \mathbb{R}^{d} \times \mathbb{R}_{d}$,

$$
\begin{aligned}
& \Im a^{\varphi}(x, \xi) \leqslant 0, \\
& \Im a^{\varphi}(x, \xi)=0 \quad \Leftrightarrow \quad(x, \xi) \in K_{0} \times(2 \pi \mathbb{Z})^{d} .
\end{aligned}
$$

(ii) For $(x, \xi) \in K_{0} \times(2 \pi \mathbb{Z})^{d}$,

$$
\begin{aligned}
\left(a^{\varphi}\right)(x, \xi) & =0, \\
\left(a^{\varphi}\right)_{\xi}^{\prime}(x, \xi) & =\left(\Re a^{\varphi}\right)_{\xi}^{\prime}(x, \xi)=H_{p}^{\prime}\left(x, \varphi^{\prime}(x)\right) \neq 0, \\
\left(a^{\varphi}\right)_{x}^{\prime}(x, \xi) & =0, \\
\left(a^{\varphi}\right)_{\xi \xi}^{\prime \prime}(x, \xi) & =-i H_{p p}^{\prime \prime}\left(x, \varphi^{\prime}(x)\right), \\
\left(a^{\varphi}\right)_{\xi x}^{\prime \prime}(x, \xi) & =H_{p x}^{\prime \prime}\left(x, \varphi^{\prime}(x)\right)+H_{p p}^{\prime \prime}\left(x, \varphi^{\prime}(x)\right) \varphi^{\prime \prime}(x), \\
\left(a^{\varphi}\right)_{x x}^{\prime \prime}(x, \xi) & =0 .
\end{aligned}
$$


(iii) $\rho:[0, \tau] \rightarrow K_{0} \times(2 \pi \mathbb{Z})^{d}$ with $\tau>0$ is an integral curve of $X_{\Re a^{\varphi}}$, if and only if, for $(x(t), \xi(t)):=\rho(t)$, the curve $[0, \tau] \ni t \mapsto\left(x(t), \varphi^{\prime}(x(t))\right)$ is a piece of an integral curve of $X_{H}$ emanating from some point on $\left\{y_{0}\right\} \times \mathbb{E}_{y_{0}} \subset T^{*} \mathbb{R}^{d}$.

(iv) Let $\gamma:[0, \tau] \rightarrow K_{0}$ denote the geodesic from $y^{\star}=\gamma(0)$ to $x^{\star}=\gamma(\tau)$ parametrized such that it is the projection onto $\mathbb{R}^{d}$ of a trajectory of $X_{H}$. Then, for every constant vector e $\in(2 \pi \mathbb{Z})^{d}$, $(\gamma, e)$ is an integral curve of $X_{\Re a^{\varphi}}$.

Proof: (i): Similarly to analogous considerations in [20] we deduce (44) and (45) from the representation

$$
\begin{array}{r}
\widetilde{V}\left(x, \xi+i \varphi^{\prime}(x)\right)=\sum_{\substack{\ell \in \mathbb{Z}^{d}: \\
0<|\ell| \leqslant R}} V(x, \ell)\left\{\cosh \left(\left\langle\ell \mid \varphi^{\prime}(x)\right\rangle\right) \cos (\langle\ell \mid \xi\rangle)\right. \\
\left.-i \sinh \left(\left\langle\ell \mid \varphi^{\prime}(x)\right\rangle\right) \sin (\langle\ell \mid \xi\rangle)\right\},
\end{array}
$$

the positivity of $V$, and the inequality $H\left(x, \varphi^{\prime}(x)\right)=\widetilde{V}\left(x, i \varphi^{\prime}(x)\right)-U(x) \leqslant 0, x \in \mathbb{R}^{d}$. In the derivation of (45) we make use of the assumption that $V(x, \ell) \geqslant 1$, if $|\ell|=1$, and the trivial fact that, for all $\xi \in \mathbb{R}_{d} \backslash(2 \pi \mathbb{Z})^{d}$, we find some $k \in \mathbb{Z}^{d},|k|=1$, such that $\cos (\langle k \mid \xi\rangle)<1$.

(ii) follows from straightforward computations. In particular, we obtain, for all $x \in K_{0}$ and $\xi \in(2 \pi \mathbb{Z})^{d}$,

$$
\left(\Re a^{\varphi}\right)_{\xi}^{\prime}(x, \xi)=\sum_{\substack{\ell \in \mathbb{Z}^{d} \\ 0<|\ell| \leqslant R}} V(x, \ell)^{t} \ell \sinh \left(\left\langle\ell \mid \varphi^{\prime}(x)\right\rangle\right)=T_{p}^{\prime}\left(x, \varphi^{\prime}(x)\right) \neq 0,
$$

To see that the terms in (53) are in fact non-zero, for $(x, \xi) \in K_{0} \times(2 \pi \mathbb{Z})^{d}$, we can refer to the strict convexity of $T(x, \cdot)$, or multiply (53) with $\varphi^{\prime}(x)$ and use again that $V(x, \ell)>0$, for $|\ell|=1$.

(iii) Let $\rho=(x, \xi):[0, \tau] \rightarrow K_{0} \times(2 \pi \mathbb{Z})^{d}$ be a trajectory of $X_{\Re a^{\varphi}}$. Then (47) implies $\dot{x}(t)=\nabla_{\xi} a^{\varphi}(\rho(t))=\nabla_{p} H\left(x(t), \varphi^{\prime}(x(t))\right)$, for all $t \in[0, \tau]$. Moreover, differentiating the identity $H\left(x, \varphi^{\prime}(x)\right)=0, x \in K_{0}$, we get $\varphi^{\prime \prime}(x) \nabla_{p} H\left(x, \varphi^{\prime}(x)\right)=-\nabla_{x} H\left(x, \varphi^{\prime}(x)\right), x \in K_{0}$. From this we infer $\frac{d}{d t} \varphi^{\prime}(x(t))=-\nabla_{x} H\left(x(t), \varphi^{\prime}(x(t))\right)$, and we see that $[0, \tau] \ni t \mapsto\left(x(t), \varphi^{\prime}(x(t))\right)$ is an integral curve of $X_{H}$. By Proposition 4.5 we know that, for every $x \in K_{0},\left(x, \varphi^{\prime}(x)\right)$ lies on an integral curve of $X_{H}$ emanating from some point on $\left\{y_{0}\right\} \times \mathbb{E}_{y_{0}}$. By the unique solvability of Hamilton's equations we conclude that $[0, \tau] \ni t \mapsto\left(x(t), \varphi^{\prime}(x(t))\right)$ is a piece of an integral curve of $X_{H}$ emanating from $\left\{y_{0}\right\} \times \mathbb{E}_{y_{0}}$.

Conversely, if $[0, \tau] \ni t \mapsto\left(x(t), \varphi^{\prime}(x(t))\right)$ is a piece of an integral curve of $X_{H}$ emanating from $\left\{y_{0}\right\} \times \mathbb{F}_{y_{0}}$, then we use (47) and (48) to check that $[0, \tau] \ni t \mapsto(x(t), e)$ is an integral curve of $X_{\Re a}$, for every $e \in(2 \pi \mathbb{Z})^{d}$.

(iv) follows from (iii) and Proposition 4.5(iv).

\section{The Hamilton-Jacobi equation}

In this section we take a first step in the construction of a parametrix for $\operatorname{Op}_{h}^{W}\left(a_{W}^{\varphi}\right)$, where $\varphi$ is the weight function constructed in Proposition 4.5 and $a_{W}^{\varphi}$ is given by Lemma 3.2. Since we shall obtain the parametrix by integrating a parametrix for the corresponding heat operator we seek for approximate solutions of the time-dependent Hamilton-Jacobi equation

$$
\partial_{t} \psi(t, x, \eta)+a^{\varphi}\left(x, \psi_{x}^{\prime}(t, x, \eta)\right)=0, \quad \psi(0, x, \eta)=\langle x \mid \eta\rangle,
$$

where $t \in[0, \infty), x \in \mathbb{R}^{d}$, and $\eta \in \mathbb{R}_{d}$. Here and $a^{\varphi}$ is given by (28) with $\phi=\varphi$. Since the symbol $a^{\varphi}$ is complex-valued we have to work with almost analytic extensions and we can only hope to solve (54) up to error terms of order $\mathcal{O}_{N}\left(|\Im \psi(t, x, \eta)|^{N}\right)$, for every $N \in \mathbb{N}$. Approximate solutions of (54) have been constructed in [15] for a class of symbols which are homogenous of degree one and in [16] for purely imaginary symbols satisfying some further assumptions. Since neither of 
these works applies directly to our situation we will explain in detail how the proofs of [15] can be adapted to fit with our hypotheses. We remark that the same class of problems is adressed in 9] where approximate solutions to the heat equation are obtained in the framework of Maslov's canonical operator theory. Approximate solutions of complex eikonal and transport equations are also derived in 22 .

This section is split in two parts: In Subsection 5.1 we extend the crucial estimates from [15] on the real Hamiltonian flow associated with $a^{\varphi}$ to our situation. The essential properties of the symbol $a^{\varphi}$ required here are that the imaginary part of $a^{\varphi}$ has a fixed sign and that the derivative of $\Re a^{\varphi}$ is non-zero if $\Im a^{\varphi}$ vanishes. Both of them are ensured by our special choice of $\varphi$ as we have already observed in Lemma 4.8. In Subsection 5.2 we construct the approximate solution $\psi$. Some basic facts about almost analytic extensions are collected in Appendix C.

\subsection{Estimates for the Hamiltonian and contact flows}

We will obtain the approximate solution, $\psi$, of (54) essentially as the generating function of the flow of a real Hamiltonian vector field associated with $a^{\varphi}$. In order to control the error terms and to assure that the imaginary part of $\psi$ has the right sign it is necessary to derive suitable estimates on the flow of this vector field. This is carried out in the present subsection by adapting the arguments of [15, where the symbol is assumed to be homogenous of degree one. The same problem has also been considered in [9. We remark that the reasoning of [15] and, hence, of this subsection works globally, too.

In the following we denote the complex coordinates in $T^{*} \mathbb{C}^{d} \cong \mathbb{C}^{d} \times \mathbb{C}_{d}$ again by $(x, \xi)=\rho$, so that $\partial_{x^{i}}=\frac{1}{2}\left(\partial_{\Re x^{i}}-i \partial_{\Im x^{i}}\right), \partial_{\bar{x}^{i}}=\frac{1}{2}\left(\partial_{\Re x^{i}}+i \partial_{\Im x^{i}}\right)$, etc. We pick almost analytic extensions of $\varphi, U$, and $V(\cdot, \ell), 0<|\ell| \leqslant R$, and denote them again by the same symbols. Plugging them into (43) we obtain an extension of $a^{\varphi}$ to $\mathbb{C}^{d} \times \mathbb{C}_{d}$ which is almost analytic with respect to the first and analytic with respect to the second variable. More precisely, $a^{\varphi}$ is a trigonometric polynomial in $\xi$ with almost analytic coefficients. We denote this extension of $a^{\varphi}$ simply by $a$ in the rest of Section 5 .

Since $a$ as well as any partial derivative of $a$ satisfies the Cauchy-Riemann differential equations on the real domain we know that $a_{\rho}^{\prime}(\rho)=a_{\Re \rho}^{\prime}(\rho), a_{\rho \rho}^{\prime \prime}(\rho)=a_{\Re \rho \rho}^{\prime \prime}(\rho)$, for every real $\rho$, and, hence, (47)-(51) still hold for $(x, \xi) \in K_{0} \times(2 \pi \mathbb{Z})^{d}$, if the derivatives are interpreted as complex derivatives. We emphasize that (44) and (45) are available only for real $(x, \xi)$.

We are going to study the flow of the real field [19]

$$
\widehat{X}_{a}:=X_{a}+\bar{X}_{a}
$$

where

$$
X_{a}:=\sum_{i=1}^{d}\left(a_{\xi_{i}}^{\prime} \partial_{x^{i}}-a_{x^{i}}^{\prime} \partial_{\xi_{i}}\right)
$$

We verify that

$$
\widehat{X}_{a}=\sum_{i=1}^{d}\left\{\Re\left(a_{\xi_{i}}^{\prime}\right) \partial_{\Re x^{i}}+\Im\left(a_{\xi_{i}}^{\prime}\right) \partial_{\Im x^{i}}-\Re\left(a_{x^{i}}^{\prime}\right) \partial_{\Re \xi_{i}}-\Im\left(a_{x^{i}}^{\prime}\right) \partial_{\Im \xi_{i}}\right\},
$$

so that the definition of $\widehat{X}_{a}$ amounts to considering $X_{a}$ as a vector field on $\mathbb{R}^{4 d}$. (If we took another almost analytic extension of $a$ then the components of the corresponding real fields would be equivalent in the sense explained in Appendix [C]) We denote the flow of $\widehat{X}_{a}$ by

$$
\kappa_{t}(\rho):=(Q(t, \rho), \Xi(t, \rho)):=\exp \left(t \widehat{X}_{a}\right)(\rho), \quad \rho \in T^{*} \mathbb{C}^{d} .
$$

On account of (55) we see that the Hamiltonian equations for $Q$ and $\Xi$ read

$$
\dot{Q}=\nabla_{\xi} a(Q, \Xi), \quad \dot{\Xi}=-\nabla_{x} a(Q, \Xi) .
$$


Due to the periodicity of $a$ in $\Re \xi$ we have, for $(t, y, \eta) \in \mathbb{R} \times T^{*} \mathbb{C}^{d}$ and $e \in(2 \pi \mathbb{Z})^{d}$,

$$
Q(t, y, \eta+e)=Q(t, y, \eta), \quad \Xi(t, y, \eta+e)=\Xi(t, y, \eta)+e .
$$

It will be convenient to extend $T^{*} \mathbb{C}^{d}$ by an extra variable, $s$, which parametrizes the action. We may view the resulting space $\mathbb{C} \times T^{*} \mathbb{C}^{d}$ as a contact manifold equipped with the contact form $d s+\sum_{i=1}^{d} \xi_{i} d x^{i}$. Then we may call the vector field

$$
\widehat{K}_{a}:=K_{a}+\bar{K}_{a},
$$

where

$$
K_{a}:=-A \partial_{s}+X_{a}, \quad A:=\left\langle\xi \mid a_{\xi}^{\prime}\right\rangle-a,
$$

the real contact field associated with $a$. Here and in the following $\langle\cdot \mid \cdot \cdot\rangle$ denotes the bilinear extension to $\mathbb{C}^{d}$ of the Euclidean scalar product on $\mathbb{R}^{d}$. The function $A$ is called the elementary action. To study the flow of $\widehat{K}_{a}$ we introduce the function $\mathfrak{S}: \mathbb{C} \times T^{*} \mathbb{C}^{d} \rightarrow \mathbb{C}$, where

$$
\mathfrak{S}(s, x, \xi):=-\Im s-\langle\Im x \mid \Re \xi\rangle, \quad(s, x, \xi) \in \mathbb{C} \times T^{*} \mathbb{C}^{d}
$$

The relationship between these objects and the ones considered in [15], where the symbol is assumed to be homogenous of degree one, can be easily seen by means of the the standard reduction from non-homogenous to homogenous symbols: Denoting the momentum variable conjugate to the extra variable $s$ by $\sigma$, we set $a^{\text {hom }}(s, x, \sigma, \xi):=\sigma a\left(x, \frac{1}{\sigma} \xi\right)$, for $(s, x, \sigma, \xi) \in T^{*} \mathbb{C}^{d+1}$ with $\sigma \neq 0$. The results of [15] apply directly to the flow of the real field $\widehat{X}_{a^{\text {hom }}}$, which is defined analogously to $\widehat{X}_{a}$. Since $\sigma$ is obviously constant along the flow lines of $\widehat{X}_{a^{\text {hom }}}$ it is easily checked that $\widehat{X}_{a^{\text {hom }}} \uparrow_{\{\sigma=1\}}$ can be identified with the real contact field $\widehat{K}_{a}$. Moreover, the function $-\langle\Im(s, x) \mid \Re(\sigma, \xi)\rangle$ used in [15] reduces to $\mathfrak{S}$, for $\sigma=1$. (A direct application of [15] would, however, give suboptimal versions of the estimates (62)-(64) below.)

Lemma. 5.1 For all compact subsets $\Omega \Subset T^{*} \mathbb{C}^{d}$, there is some $C_{\Omega} \in(0, \infty)$, such that, for all $(s, \rho) \in \mathbb{C} \times \Omega$,

$$
\widehat{K}_{a}(\mathfrak{S})(s, \rho) \geqslant-\frac{1}{2} \Im a(\Re \rho)-C_{\Omega}|\Im \rho|^{3} .
$$

Proof: Taylor expanding at $\Re \rho$ in the third and fourth step, we get

$$
\begin{aligned}
& \widehat{K}_{a}(\Im s+\langle\Im x \mid \Re \xi\rangle) \\
& \quad=\Im\left(a(\rho)-\left\langle\xi \mid a_{\xi}^{\prime}(\rho)\right\rangle+\left\langle\xi-i \Im \xi \mid a_{\xi}^{\prime}(\rho)\right\rangle\right)-\left\langle\Im x \mid \Re a_{x}^{\prime}(\rho)\right\rangle \\
& =\Im a(\rho)-\left\langle\Re a_{\rho}^{\prime}(\rho) \mid \Im \rho\right\rangle \\
& =\Im a(\Re \rho)+\frac{1}{2}\left\langle\Im a_{\rho \rho}^{\prime \prime}(\Re \rho) \Im \rho \mid \Im \rho\right\rangle+\mathcal{O}\left(|\Im \rho|^{3}\right) \\
& =\frac{3}{4} \Im a(\Re \rho)+\frac{1}{8}(\Im a(\Re \rho+2 \Im \rho)+\Im a(\Re \rho-2 \Im \rho))+\mathcal{O}\left(|\Im \rho|^{3}\right) \\
& \quad \leqslant \frac{3}{4} \Im a(\Re \rho)+\mathcal{O}\left(|\Im \rho|^{3}\right) .
\end{aligned}
$$

Remark. 5.2 Exactly as in [15, §1], we may check that $\mathfrak{S}$ is invariantly defined modulo $\mathcal{O}\left(|\Im(x, \xi)|^{3}\right)$ on compact subsets with respect to coordinate changes $x \mapsto \tilde{x}$. (Of course, the covariant variables $\xi$ have to be transformed accordingly.) Then the arguments of [15, Remark 1.8] directly imply that Estimate (58) does not depend on the particular coordinate system.

In the following we fix some $\rho_{0}=\left(x_{0}, \xi_{0}\right) \in T^{*} \mathbb{R}^{d}$. We assume that there exists some complex neighbourhood, $\mathscr{V} \subset \mathbb{C}^{d}$, of $x_{0}$ and an almost analytic function, $\psi_{0} \in C^{\infty}(\mathscr{V})$ such that $\Im \psi_{0} \geqslant 0$ 
on $\mathscr{V} \cap \mathbb{R}^{d}, \Im \psi_{0}\left(x_{0}\right)=0$, and $\psi_{0}^{\prime}\left(x_{0}\right)=\xi_{0}$. By a calculation similar to (59) we then see that, for every real, compact subset $\mathscr{V}_{\mathbb{R}}^{\prime} \Subset \mathscr{V} \cap \mathbb{R}^{d}$, we find some compact $\mathscr{V}^{\prime} \Subset \mathscr{V}$ and some $C \in(0, \infty)$, such that $\mathscr{V}^{\prime} \cap \mathbb{R}^{d}=\mathscr{V}_{\mathbb{R}}^{\prime}$ and

$$
\Im \psi_{0}(x)-\left\langle\Im x \mid \Re \psi_{0}^{\prime}(x)\right\rangle \geqslant-C|\Im x|^{3}, \quad x \in \mathscr{V}^{\prime} .
$$

We introduce the set

$$
\mathfrak{L}_{0}:=\left\{\left(-\psi_{0}(x), x, \psi_{0}^{\prime}(x)\right): x \in \mathscr{V}\right\},
$$

which we may call an almost analytic Legendre submanifold. (In our applications below we will only consider the case where $\psi_{0}(x):=\psi(0, x, \eta):=\langle x \mid \eta\rangle$, for some real $\eta \in \mathbb{R}_{d}$.) Furthermore, we assume that there is some $\tau \geqslant 0$ such that $\kappa_{t}\left(\rho_{0}\right)=\exp \left(t \widehat{X}_{a}\right)\left(\rho_{0}\right)$ is real, for all $t \in[0, \tau]$. If $\tau>0$, we additionally assume that $\Im a\left(\rho_{0}\right)=0$. In this case it holds $(\Im a)^{\prime}\left(\kappa_{t}\left(\rho_{0}\right)\right)=0$, and, hence, $\Im a\left(\kappa_{t}\left(\rho_{0}\right)\right)=0$, for $t \in[0, \tau]$. By (47) we also know that $(\Re a)^{\prime}\left(\kappa_{t}\left(\rho_{0}\right)\right) \neq 0$, for $t \in[0, \tau]$. Denoting the flow of $\widehat{K}_{a}$ by

$$
\Phi_{t}:=\exp \left(t \widehat{K}_{a}\right)
$$

we have that

$$
\Phi_{t}(s, \rho)=\left(\varsigma_{t}(s, \rho), \kappa_{t}(\rho)\right), \quad \varsigma_{t}(s, \rho):=s-\int_{0}^{t} A\left(\kappa_{r}(\rho)\right) d r
$$

In particular, $\Phi_{t}\left(-\psi_{0}\left(x_{0}\right), \rho_{0}\right)$ is also real, for $t \in[0, \tau]$.

Lemma. 5.3 In the situation described above there exist $\varepsilon>0, C \in(0, \infty)$, and some neighbourhood $\mathscr{O} \subset \mathbb{C} \times T^{*} \mathbb{C}^{d}$ of $\left(-\psi_{0}\left(x_{0}\right), \rho_{0}\right)$ such that the following inequalities hold on $\mathfrak{L}_{0} \cap \mathscr{O}$, for all $0 \leqslant r \leqslant t \leqslant \tau+\varepsilon$,

$$
\begin{aligned}
\mathfrak{S}\left(\Phi_{t}\right) & \geqslant-\frac{1}{3} \int_{0}^{t} \Im a\left(\Re \kappa_{u}\right) d u-C\left|\Im \kappa_{t}\right|^{3} \\
\left|\Im \kappa_{t}\right|^{2}+\mathfrak{S}\left(\Phi_{t}\right) & \geqslant \frac{1}{C}\left\{\left|\Im \kappa_{r}\right|^{2}+\mathfrak{S}\left(\Phi_{r}\right)-\int_{r}^{t} \Im a\left(\Re \kappa_{u}\right) d u\right\} \\
\left|\Im \kappa_{r}\right|^{2} & \leqslant C\left(\left|\Im \kappa_{t}\right|-\Im \varsigma_{t}\right) .
\end{aligned}
$$

In particular, if $(s, \rho) \in \mathfrak{L}_{0} \cap \mathscr{O}$ and $\kappa_{t}(\rho)$ and $\varsigma_{t}(s, \rho)$ are both real, for some $t \in[0, \tau+\varepsilon]$, then $\kappa_{r}(\rho)$ is real for all $r \in[0, t]$.

PRoOF: Taylor expanding the right side of $\frac{d}{d t} \Im \kappa_{t}=\Im \widehat{X}_{a}\left(\kappa_{t}\right)$ at $\Re \kappa_{t}$ and using Duhamel's formula we obtain, exactly as in [15, pp. 351], the estimate

$$
\left|\Im \kappa_{u}\right| \leqslant \mathcal{O}(1)\left(\left|\Im \kappa_{t}\right|+\int_{u}^{t}\left|\Im a^{\prime}\left(\Re \kappa_{r}\right)\right| d r\right)
$$

It holds for all $\rho$ in a compact, complex neighbourhood of $\rho_{0}$ and $u, t \in\left[0, \tau+\varepsilon_{1}\right]$, for some $\varepsilon_{1}>0$. Since, for $\rho$ in a compact set, the curves $\left[0, \tau+\varepsilon_{1}\right] \ni t \mapsto \Re \kappa_{t}(\rho)$ stay in a compact set, we may apply the standard estimate for positive functions to (65), which together with Hölder's inequality gives

$$
\left|\Im \kappa_{r}\right| \leqslant \mathcal{O}(1)\left(\left|\Im \kappa_{t}\right|+\left(\int_{u}^{t}-\Im a\left(\Re \kappa_{v}\right) d v\right)^{1 / 2}\right)
$$

for $0 \leqslant u \leqslant r \leqslant t \leqslant \tau+\varepsilon_{1}$. Next, we integrate the estimate (다) for $\frac{d}{d t} \mathfrak{S}\left(\Phi_{t}\right)=\widehat{K}_{a}(\mathfrak{S})\left(\Phi_{t}\right)$ from $u$ to $t$, use (66) to bound $\left|\Im \kappa_{r}\right|, r \in[u, t]$, and arrive at

$$
\mathfrak{S}\left(\Phi_{t}\right) \geqslant \mathfrak{S}\left(\Phi_{u}\right)-\frac{1}{2} \int_{u}^{t} \Im a\left(\kappa_{r}\right) d r-\mathcal{O}(1)(t-u)\left|\Im \kappa_{t}\right|^{3}-\left(\int_{u}^{t}-\Im a\left(\Re \kappa_{r}\right) d r\right)^{3 / 2} .
$$


For $u=0$, (60) implies

$$
\mathfrak{S}\left(\Phi_{0}\left(-\psi_{0}(x), x, \psi_{0}^{\prime}(x)\right)\right) \geqslant-\mathcal{O}(1)|\Im x|^{3},
$$

for $x$ in a sufficiently small neighbourhood of $x_{0}$. Here the last term can again be estimated by means of (66). We further notice that we can make the integral appearing in (67) arbitrarily small by assuming that $\rho$ is contained in a sufficiently small neighbourhood of $\rho_{0}$, and that $\varepsilon_{1}>0$ is sufficiently small. For, if $t \in[0, \tau]$, the integral vanishes at $\rho_{0}$. Setting $u=0$ we thus get (62). The proofs of (63) and (64) are identical to those in [15, pp. 355], except for Equation (3.30) in [15, where $-\Im \varsigma_{t}$ has to be added on the right side.

\subsection{Approximate solution of the Hamilton-Jacobi equation}

The aim of this subsection is to construct an approximate solution, $\psi$, of (54). We will define $\psi$ by a formula well-known from classical mechanics and proceed along traditional arguments to check that $\psi$ has the required properties. In doing so we use Lemma 5.3 to controll the error terms and to ensure that $\Im \psi$ is positive. Since Lemma 5.3 is applicable near real integral curves of $\widehat{X}_{a}$, we start by considering the latter more closely. All the time we keep on using the notation of Proposition 4.5 with $H=T-U$.

Lemma. 5.4 Let $\tau>0$ and $\rho:[0, \tau] \rightarrow T^{*} \mathbb{R}^{d}$ be a real integral curve of $\widehat{X}_{a}$ with $\rho(0) \in\{\Im a=0\}$. Then $\rho([0, \tau]) \subset K_{0} \times(2 \pi \mathbb{Z})^{d}$. If we write $\rho(t)=(x(t), \xi(t))$, then $[0, \tau] \ni t \mapsto\left(x(t), \varphi^{\prime}(x(t))\right)$ is a piece of an integral curve of $X_{H}$ emanating from some point on $\left\{y_{0}\right\} \times \mathbb{E}_{y_{0}} \subset T^{*} \mathbb{R}^{d}$. Conversely, every point of $K_{0}$ lies on the projection of an integral curve of $X_{H}$ emanating from some point on $\left\{y_{0}\right\} \times \mathbb{E}_{y_{0}}$ and the piece of that projection inside $K_{0}$ is the projection of a real integral curve of $\widehat{X}_{a}$ with momentum $e \in(2 \pi \mathbb{Z})^{d}$.

Proof: $\rho$ being a real integral curve of $\widehat{X}_{a}$ means $\Im\left(\begin{array}{c}\nabla_{\xi} a \\ -\nabla_{x} a\end{array}\right)(\rho(t))=\frac{d}{d t} \Im \rho(t)=0$. Since $a$ fulfills the Cauchy-Riemann differential equations on the real domain, it follows that $(\Im a)_{\Re \rho}^{\prime}(\rho(t))=$ $\Im\left(a_{\rho}^{\prime}\right)(\rho(t))=0$. Hence, the derivative of $\left.(\Im a)\right|_{T^{*} \mathbb{R}^{d}}$ vanishes along $\rho$ and $\Im a(\rho(0))=0$ thus implies $\rho([0, \tau]) \subset\{\Im a=0\}$. Using (45) we conclude $\rho([0, \tau]) \subset K_{0} \times(2 \pi \mathbb{Z})^{d}$. The remaining assertions follows from Proposition 4.5] and Lemma 4.8(iii).

Of course, there might be real integral curves of $\widehat{X}_{a}$ defined on a non-trivial interval running in a region where $\Im a<0$. These do, however, not play any important role since the imaginary part of the action is strictly decreasing along them.

The approximate solution $\psi$ will turn out to be "almost" a generating function of the canonical relations

$$
C_{t}=\left\{(x, \xi, y, \eta) \in T^{*} \mathbb{C}^{d} \times T^{*} \mathbb{C}^{d}:(x, \xi)=\kappa_{t}(y, \eta)\right\}, \quad t \geqslant 0 .
$$

We recall our notation $\kappa_{t}=\exp \left(t \widehat{X}_{a}\right)$. For every $t \geqslant 0$, the relation $C_{t}$ is $2 \pi$-periodic in the sense that $(x, \xi, y, \eta) \in C_{t}$ implies $(x, \xi+e, y, \eta+e) \in C_{t}$, for every $e \in(2 \pi \mathbb{Z})^{d}$. To study $C_{t}$ we denote the bilinear extension of the canonical symplectic form on $T^{*} \mathbb{R}^{d}$ to $T^{*} \mathbb{C}^{d}$ by $\widetilde{\sigma}$, i.e.

$$
\widetilde{\sigma}\left(\vartheta, \vartheta^{\prime}\right)={ }^{t} \vartheta\left(\begin{array}{cc}
0 & -\mathbb{1} \\
\mathbb{1} & 0
\end{array}\right) \vartheta^{\prime}, \quad \vartheta, \vartheta^{\prime} \in T T^{*} \mathbb{C}^{d}
$$

Furthermore, we write

$$
\widetilde{\sigma} \ominus \widetilde{\sigma}\left(\vartheta, \vartheta^{\prime}\right):=\widetilde{\sigma}\left(\left(\vartheta_{x}, \vartheta_{\xi}\right),\left(\vartheta_{x}^{\prime}, \vartheta_{\xi}^{\prime}\right)\right)-\widetilde{\sigma}\left(\left(\vartheta_{y}, \vartheta_{\eta}\right),\left(\vartheta_{y}^{\prime}, \vartheta_{\eta}^{\prime}\right)\right),
$$

where $\vartheta=\left(\vartheta_{x}, \vartheta_{\xi}, \vartheta_{y}, \vartheta_{\eta}\right) \in T\left(T^{*} \mathbb{C}^{d}\right) \times T\left(T^{*} \mathbb{C}^{d}\right)$ and similarly for $\vartheta^{\prime}$.

Lemma. 5.5 Let $\tau>0$ and $\rho:[0, \tau] \rightarrow K_{0} \times(2 \pi \mathbb{Z})^{d}$ be a real integral curve of $\widehat{X}_{a}$. Then $C_{t}$ is strictly positive along $\rho$, that is, for all $t \in[0, \tau]$,

$$
\frac{1}{2 i} \widetilde{\sigma} \ominus \widetilde{\sigma}(\vartheta, \bar{\vartheta}) \geqslant 0, \quad \vartheta \in T_{(\rho(t), \rho(0))} C_{t},
$$


with equality if and only if $\vartheta$ is contained in the complexification of the tangent space at $(\rho(t), \rho(0))$ of $C_{t} \cap \mathbb{R}^{d}$. The latter condition holds, if and only if $\vartheta=\left(\vartheta_{x}, 0, \vartheta_{y}, 0\right)$.

PROOF: Along the real curve $\rho(t)=:(x(t), e) \in K_{0} \times(2 \pi \mathbb{Z})^{d}$ the fundamental matrix of $a$ is given by

$$
\mathbb{F}_{a}(x(t), e)=\left(\begin{array}{cc}
a_{\xi x}^{\prime \prime} & a_{\xi \xi}^{\prime \prime} \\
-a_{x x}^{\prime \prime} & -a_{x \xi}^{\prime \prime}
\end{array}\right)(x(t), e)=\left(\begin{array}{cc}
\mathbb{B}(t) & -i \mathbb{A}(t) \\
0 & -{ }^{t} \mathbb{B}(t)
\end{array}\right)
$$

where

$$
\mathbb{A}(t):=H_{p p}^{\prime \prime}\left(x(t), \varphi^{\prime}(x(t))\right), \quad \mathbb{B}(t):=H_{p x}^{\prime \prime}\left(x(t), \varphi^{\prime}(x(t))\right)+\mathbb{A}(t) \varphi^{\prime \prime}(x(t)),
$$

for $t \in[0, \tau]$. It holds $\vartheta=(\theta(t), \theta(0))=\left(\theta_{x}(t), \theta_{\xi}(t), \theta_{x}(0), \theta_{\xi}(0)\right)$, where $\theta(t)=\left(\theta_{x}(t), \theta_{\xi}(t)\right)=$ $\kappa_{t}^{\prime}(\rho(0)) \theta(0), t \in[0, \tau]$, for some $\theta(0) \in T_{\rho(0)} T^{*} \mathbb{C}^{d}$, and we know that $\theta(t)$ satisfies $\frac{d}{d t} \theta(t)=$ $\mathbb{F}_{a}(\rho(t)) \theta(t)$, for $t \in[0, \tau]$. We thus obtain

$$
\frac{d}{d t} \frac{1}{2 i} \tilde{\sigma}(\theta(t), \overline{\theta(t)})=\left\langle\Re \theta_{\xi}(t) \mid \mathbb{A}(t) \Re \theta_{\xi}(t)\right\rangle+\left\langle\Im \theta_{\xi}(t) \mid \mathbb{A}(t) \Im \theta_{\xi}(t)\right\rangle,
$$

for $t \in[0, \tau]$. Integrating the previous identity from 0 to $\tau$ and using the fact that $\mathbb{A}(t)$ is positive definite, for all $t \in[0, \tau]$, we see that assertion of the lemma holds true. In fact, the structure of $\mathbb{F}_{a}(x(t), e)$ shows that we have equality in (68) if and only if $\theta_{\xi}(0)=0$.

In the following we set

$$
\begin{aligned}
\widetilde{\mathscr{D}} & :=\left\{(t, y, e) \in[0, \infty) \times K_{0} \times(2 \pi \mathbb{Z})^{d}: \quad \kappa_{t^{\prime}}(y, e) \in K_{0} \times\{e\}, t^{\prime} \in[0, t]\right\}, \\
\mathscr{D} & :=\left\{(t, x, e) \in[0, \infty) \times K_{0} \times(2 \pi \mathbb{Z})^{d}: \quad \kappa_{-t^{\prime}}(x, e) \in K_{0} \times\{e\}, t^{\prime} \in[0, t]\right\}, \\
\widetilde{\mathscr{E}} & :=\left(\{0\} \times \mathbb{R}^{d} \times \mathbb{R}_{d}\right) \cup \widetilde{D}, \quad \mathscr{E}:=\left(\{0\} \times \mathbb{R}^{d} \times \mathbb{R}_{d}\right) \cup \mathscr{D} .
\end{aligned}
$$

By Proposition 4.5 and Lemma [5.4 it is clear that there is some $t_{0} \in(0, \infty)$ such that $\widetilde{D}, \mathscr{D} \subset$ $\left[0, t_{0}\right] \times K_{0} \times(2 \pi \mathbb{Z})^{d}$ and, moreover,

$$
\forall(t, y, e) \in \widetilde{\mathscr{D}}: \quad \dot{Q}(t, y, e)=\nabla_{p} H\left(Q(t, y, e), \varphi^{\prime}(Q(t, y, e))\right) .
$$

Lemma. 5.6 There is some neighbourhood, $\mathscr{G}$, of $\mathscr{E}$ in $[0, \infty) \times \mathbb{C}^{d} \times \mathbb{C}_{d}$ with the following properties: There are two smooth functions, $k$ and $g$, defined on $\mathscr{G}$, such that, for every $(t, x, \xi, y, \eta) \in$ $[0, \infty) \times T^{*} \mathbb{C}^{d} \times T^{*} \mathbb{C}^{d}$,

$$
(x, \xi, y, \eta) \in C_{t} \quad \Leftrightarrow \quad y=k(t, x, \eta) \wedge \xi=g(t, x, \eta) .
$$

$\mathscr{G}$ is $2 \pi$-periodic in $\Re \eta$ and, for all $(t, x, \eta) \in \mathscr{G}$ and $e \in(2 \pi \mathbb{Z})^{d}$,

$$
g(t, x, \eta+e)=g(t, x, \eta)+e, \quad k(t, x, \eta+e)=k(t, x, \eta) .
$$

Moreover,

$$
\forall(t, x, e) \in \mathscr{D}: \quad g(t, x, e)=e, \quad k(t, x, e)=\pi \exp \left(-t X_{H}\right)\left(x, \varphi^{\prime}(x)\right) .
$$

ProOF: It suffices to show that the projection $C_{t} \ni(x, \xi, y, \eta) \mapsto(x, \eta)$ is bijective in a neighbourhood of each point on $C_{t}$ with $(t, x, \eta) \in \mathscr{E}$. For $t=0$, this is trivial by definition of $C_{0}$. So, it remains to treat the case $(t, x, \eta) \in \mathscr{D}, t>0$.

Let $t>0$ and let $\rho:[0, t] \rightarrow K_{0} \times(2 \pi \mathbb{Z})^{d}$ be a real integral curve of $\widehat{X}_{a}$. Let $\vartheta=$ $\left(\vartheta_{x}, \vartheta_{\xi}, \vartheta_{y}, \vartheta_{\eta}\right) \in T_{(\rho(t), \rho(0))} C_{t}$ be such that $\vartheta_{x}=0$ and $\vartheta_{\eta}=0$. It clearly holds $\widetilde{\sigma} \ominus \widetilde{\sigma}(\vartheta, \bar{\vartheta})=0$. Using the same notation as in the proof of Lemma 5.5 we thus have that $\theta_{\xi}(r)=0$, for all $r \in[0, t]$. In particular, $\vartheta_{\xi}=0$. Then it is also clear that $\vartheta_{y}=0$, because $\theta_{x}(r)$ solves the initial value problem $\frac{d}{d r} \theta_{x}(r)=\mathbb{B}(r) \theta_{x}(r), \theta_{x}(t)=0$. Consequently, the projection $C_{t} \ni(x, \xi, y, \eta) \mapsto(x, \eta)$ is bijective in a neighbourhood of $(\rho(t), \rho(0)) \in C_{t}$. 
Finally, (71) follows from the periodicity of $C_{t}$ and (72) from Lemma 5.4 and the definition of $\mathscr{D}$.

In the remaining part of this section we show that an approximate solution of (54) is given by

$$
\psi(t, x, \eta):=\langle k(t, x, \eta) \mid \eta\rangle+\int_{0}^{t} A\left(\kappa_{r}(k(t, x, \eta), \eta)\right) d r, \quad(t, x, \eta) \in \mathscr{G} .
$$

We observe that $\psi(0, x, \eta)=\langle x \mid \eta\rangle$ and a direct computation using (56), (577), and (71) shows, for $(t, x, \eta) \in \mathscr{G}$ and $e \in(2 \pi \mathbb{Z})^{d}$,

$$
\psi(t, x, \eta+e)=\psi(t, x, \eta)+\langle\eta \mid e\rangle,
$$

so that the difference $\psi-\langle x \mid \eta\rangle$ is $(2 \pi \mathbb{Z})^{d}$-periodic in $\Re \eta$. By definition of $\mathscr{E}$ and (45) and (47) we also know that

$$
\forall(t, x, \eta) \in \mathscr{E}: \quad \Im \psi(t, x, \eta)=0 .
$$

To study $\psi$ further we introduce, for $(t, y, \eta) \in \mathbb{R} \times T^{*} \mathbb{C}^{d}$,

$$
\begin{aligned}
& Z(t, y, \eta):=-\varsigma_{t}(-\langle y \mid \eta\rangle, y, \eta)=\langle y \mid \eta\rangle+\int_{0}^{t} A\left(\kappa_{r}(y, \eta)\right) d r \\
& \Theta(t, y, \eta):=\left(t, \kappa_{t}(y, \eta)\right), \\
& \widetilde{\Gamma}(t, y, \eta):=|\Im(Q, \Xi)(t, y, \eta)|^{2}+\mathfrak{S}(-Z, Q, \Xi)(t, y, \eta) .
\end{aligned}
$$

Lemma. 5.7 There is a small neighbourhood, $\widetilde{\mathscr{N}}$, of $\widetilde{\mathscr{E}}$ in $[0, \infty) \times \mathbb{C}^{d} \times \mathbb{R}_{d}$, which is $(2 \pi \mathbb{Z})^{d}$ periodic in the last variable, such that, for all $(t, y, \eta) \in \widetilde{\mathscr{N}}$ and $r \in[0, t]$,

$$
\begin{aligned}
\Im Z(t, y, \eta) \geqslant & \langle\Im Q(t, y, \eta) \mid \Re \Xi(t, y, \eta)\rangle-\frac{1}{3} \int_{0}^{t} \Im a\left(\Re \kappa_{u}(y, \eta)\right) d u \\
& -\mathcal{O}(1)|\Im(Q, \Xi)(t, y, \eta)|^{3} \\
\frac{1}{2}|\Im(Q, \Xi)(r, y, \eta)|^{2} \leqslant & \widetilde{\Gamma}(r, y, \eta) \leqslant \mathcal{O}(1) \widetilde{\Gamma}(t, y, \eta)
\end{aligned}
$$

where the $\mathcal{O}$-symbols are uniform on compact subsets of $\widetilde{\mathscr{N}}$.

Proof: For each fixed $\eta \in \mathbb{R}_{d}$, we apply Lemma 5.3 with $\psi_{0}(y):=\langle y \mid \eta\rangle, y \in \mathbb{C}^{d}$, and $\rho_{0} \in \mathbb{R}^{d} \times\{\eta\}$. If $\rho_{0} \in K_{0} \times(2 \pi \mathbb{Z})^{d}$, we choose $\tau:=\max \left\{t>0: \kappa_{t}\left(\rho_{0}\right) \in K_{0} \times(2 \pi \mathbb{Z})^{d}\right\}$. If $\rho_{0} \notin K_{0} \times(2 \pi \mathbb{Z})^{d}$, we set $\tau=0$. So, if $\tau>0$, then Lemma 5.4 ensures that $\kappa_{t}\left(\rho_{0}\right)$ is real, for $t \in[0, \tau]$, and by (45) we know that $\Im a\left(\rho_{0}\right)=0$. Then (76) follows from (62). The first inequality in (77) follows from (44), (76), and the fact that we can assume $|\Im(Q, \Xi)(r, y, \eta)|$ to be arbitrarily small by restricting our attention to some sufficiently small neighbourhood of $\widetilde{E}$, where $|\Im(Q, \Xi)(r, y, \eta)|$ vanishes. The second inequality in (77) follows from (44) and (63).

In the next lemma we compare the differential of $Z$ with the pull-back under $\Theta$ of the Cartan form,

$$
\omega:=\xi d x-a(x, \xi) d t
$$

considered as a form on $\mathbb{R} \times T^{*} \mathbb{C}^{d}$. Following a standard proof known from classical mechanics (see, e.g., [6, pp. 479]) we only have to keep track of the error terms.

Lemma. 5.8 (i) On every compact subset $\Omega \Subset \mathbb{R} \times T^{*} \mathbb{C}^{d}$ such that $|t| \leqslant t_{0}$ on $\Omega$,

$$
d Z=-\Theta^{*} \omega+y d \eta+\mathcal{O}_{N}\left(\max _{|\tilde{t}| \leqslant t_{0}} \widetilde{\Gamma}(\tilde{t}, y, \eta)^{N}\right) .
$$


(ii) Let $\widetilde{\mathscr{N}}$ be as in Lemma 5.7. Then

$$
d Z=-\Theta^{*} \omega+y d \eta+t \mathcal{O}_{N}\left(\widetilde{\Gamma}^{N}\right)
$$

on $\widetilde{\mathscr{N}}$, where the $\mathcal{O}_{N}$-symbols are uniform on compact subsets of $\widetilde{\mathscr{N}}$.

Proof: We set $\lambda:=d Z+\Theta^{*} \omega$ and use

$$
\dot{Z}=\left\langle\Xi \mid a_{\xi}^{\prime}(Q, \Xi)\right\rangle-a(Q, \Xi)=\langle\Xi \mid \dot{Q}\rangle-a(Q, \Xi)
$$

to obtain

$$
\lambda=\left(Z_{y}^{\prime}-\left\langle\Xi \mid Q_{y}^{\prime}\right\rangle\right) d y+\left(Z_{\bar{y}}^{\prime}-\left\langle\Xi \mid Q_{\bar{y}}^{\prime}\right\rangle\right) d \bar{y}+\left(Z_{\eta}^{\prime}-\left\langle\Xi \mid Q_{\eta}^{\prime}\right\rangle\right) d \eta+\left(Z_{\bar{\eta}}^{\prime}-\left\langle\Xi \mid Q_{\bar{\eta}}^{\prime}\right\rangle\right) d \bar{\eta} .
$$

Now, let $\alpha$ denote any of the variables $y^{i}, \bar{y}^{i}, \eta_{i}, \bar{\eta}_{i}, 1 \leqslant i \leqslant d$, and set $\lambda_{\alpha}:=\left(Z_{\alpha}^{\prime}-\left\langle\Xi \mid Q_{\alpha}^{\prime}\right\rangle\right)$. Using successively (79), the Hamiltonian equations (56) and the almost analyticity of $a$ we find

$$
\begin{aligned}
\dot{\lambda}_{\alpha} & =\partial_{\alpha}(\langle\Xi \mid \dot{Q}\rangle-a(Q, \Xi))-\left\langle\dot{\Xi} \mid Q_{\alpha}^{\prime}\right\rangle-\left\langle\Xi \mid \dot{Q}_{\alpha}^{\prime}\right\rangle \\
& =\left\langle\Xi_{\alpha}^{\prime} \mid a_{\xi}^{\prime}(Q, \Xi)\right\rangle-\partial_{\alpha}(a(Q, \xi))+\left\langle a_{x}^{\prime}(Q, \Xi) \mid Q_{\alpha}^{\prime}\right\rangle \\
& =-a_{\bar{x}}^{\prime}(Q, \Xi) \overline{Q_{\bar{\alpha}}^{\prime}}-a_{\bar{\xi}}^{\prime}(Q, \Xi) \overline{\Xi_{\alpha}^{\prime}} \\
& =\mathcal{O}_{N}\left(|\Im(Q, \Xi)|^{N}\right) .
\end{aligned}
$$

Due to the initial conditions $\left.(Z, Q, \Xi)\right|_{t=0}=(\langle y \mid \eta\rangle, y, \eta)$ we have

$\left.Z_{y}^{\prime}\right|_{t=0}=\eta,\left.\quad Z_{\eta}^{\prime}\right|_{t=0}=y,\left.\quad Z_{\bar{y}}^{\prime}\right|_{t=0}=\left.Z_{\bar{\eta}}^{\prime}\right|_{t=0}=0,\left.\quad Q_{y}^{\prime}\right|_{t=0}=\mathbb{1},\left.\quad Q_{\eta}^{\prime}\right|_{t=0}=\left.Q_{\bar{y}}^{\prime}\right|_{t=0}=\left.Q_{\bar{\eta}}^{\prime}\right|_{t=0}=0$.

We conclude

$$
\lambda(t, y, \eta)=y d \eta+\int_{0}^{t} \mathcal{O}_{N}\left(|\Im(Q, \Xi)(r, y, \eta)|^{N}\right) d r,
$$

which implies (i). (ii) follows from Lemma 5.7. (Note that (777) is available only for real $\eta$.)

In what follows we set

$$
K(t, x, \eta):=(t, k(t, x, \eta), \eta)
$$

for all $(t, x, \eta) \in \mathscr{G}$, so that

$$
K^{*} Z=Z \circ K=\psi, \quad K^{*} Q=Q \circ K=x, \quad K^{*} \Xi=\Xi \circ K=g .
$$

Furthermore, we write $\mathscr{N}:=K^{-1}(\widetilde{\mathscr{N}})$ and

$$
\Gamma:=K^{*} \widetilde{\Gamma}=\widetilde{\Gamma} \circ K=|\Im(x, g)|^{2}-\langle\Im x \mid \Re g\rangle+\psi .
$$

The set $\mathscr{N} \subset \mathscr{G}$ is a neighbourhood of $\mathscr{E}$ in $[0, \infty) \times \mathbb{C}^{d} \times \mathbb{R}_{d}$, which is $2 \pi$-periodic in $\eta$.

Proposition. 5.9 Let $\psi$ be given by (73). Then

$$
\begin{aligned}
\partial_{(t, x, \bar{x}, \Re \eta)}^{\alpha}\left(\partial_{t} \psi+a\left(x, \psi_{x}^{\prime}\right)\right) & =\mathcal{O}_{N, \alpha}\left(\Gamma^{N}\right), \\
\partial_{(t, x, \bar{x}, \Re \eta)}^{\alpha}\left(\psi_{x}^{\prime}-g\right) & =\mathcal{O}_{N, \alpha}\left(\Gamma^{N}\right), \\
\partial_{(t, x, \bar{x}, \Re \eta)}^{\alpha}\left(\psi_{\eta}^{\prime}-k\right) & =\mathcal{O}_{N, \alpha}\left(\Gamma^{N}\right), \\
\partial_{(t, x, \bar{x} \Re \eta)}^{\alpha} \psi_{\bar{x}}^{\prime} & =\mathcal{O}_{N, \alpha}\left(\Gamma^{N}\right), \\
\partial_{(t, x, \bar{x}, \Re \eta)}^{\alpha} \psi_{\bar{\eta}}^{\prime} & =\mathcal{O}_{N, \alpha}\left(\Gamma^{N}\right),
\end{aligned}
$$

on $\mathscr{N}$, for $N \in \mathbb{N}$ and every multi-index $\alpha \in \mathbb{N}_{0}^{3 d+1}$. All $\mathcal{O}_{N, \alpha}$-symbols are uniform on compact subsets of $\mathscr{N}$. 
Proof: On account of Lemma 5.8(ii) we obtain

$$
\begin{aligned}
d \psi & =d\left(K^{*} Z\right)=K^{*}(d Z) \\
& =K^{*}\left(\Theta^{*} \omega+y d \eta+t \mathcal{O}_{N}\left(\widetilde{\Gamma}^{N}\right)\right) \\
& =g d x-a(x, g) d t+k d \eta+t \mathcal{O}_{N}\left(\Gamma^{N}\right)
\end{aligned}
$$

on $\mathscr{N}$. Using Lemma C.1 we infer that (81) -

The next corollary summarizes the properties of $\psi$ on the real domain, where the weight $\Gamma$ can actually be replaced by $\Im \psi$ :

Corollary. $\mathbf{5 . 1 0}$ (i) There is some neighbourhood, $\mathscr{M}_{\mathbb{R}}$, of $\mathscr{E}$ in $[0, \infty) \times \mathbb{R}^{d} \times \mathbb{R}_{d}$, which is $2 \pi$ periodic in the last variable, such that, for all $(t, x, \eta) \in \mathscr{M}_{\mathbb{R}}$,

$$
\begin{aligned}
& \Im \psi(t, x, \eta) \geqslant \frac{1}{\mathcal{O}(1)}|\Im g(t, x, \eta)|^{2}, \\
& \Im \psi(t, x, \eta)=0 \Leftrightarrow(t, x, \eta) \in \mathscr{E} .
\end{aligned}
$$

(Here the $\mathcal{O}$-symbol is again uniform on compact subsets of $\mathscr{M}_{\mathbb{R}}$.) Consequently,

$$
\Im \psi \geqslant \frac{1}{\mathcal{O}(1)} \Gamma \quad \text { on } \mathscr{M}_{\mathbb{R}}
$$

so that (81)-(85) hold true on $\mathscr{M}_{\mathbb{R}}$ with the right sides replaced by $\mathcal{O}_{N}\left((\Im \psi)^{N}\right)$. In particular,

$$
\partial_{(t, x, \bar{x}, \Re \eta)}^{\alpha}\left(\partial_{t} \psi+a\left(x, \psi_{x}^{\prime}\right)\right)=\mathcal{O}_{N, \alpha}\left((\Im \psi)^{N}\right) \quad \text { on } \mathscr{M}_{\mathbb{R}}
$$

(ii) For all $(t, x, e) \in \mathscr{D}$,

$$
\partial_{t} \psi(t, x, e)=0, \quad \nabla_{\eta} \psi(t, x, e)=\pi \exp \left(-t X_{H}\right)\left(x, \varphi^{\prime}(x)\right), \quad \psi_{x}^{\prime}(t, x, e)=e .
$$

ProOF: (i) We observe that (44), (766), and (80) imply

$$
\Im \psi-\langle\Im x \mid \Re g\rangle \geqslant-\mathcal{O}(1)|\Im(x, g)|^{3} \quad \text { on } \mathscr{N} .
$$

Let $\left(t_{0}, x_{0}, \eta_{0}\right) \in \mathscr{E}$. From (72) we know that $\Im g$ vanishes on $\mathscr{E}$ and we can thus find some compact, real neighbourhood, $K^{\prime}$, of $\left(t_{0}, x_{0}, \eta_{0}\right)$ in $\mathscr{N} \cap\left([0, \infty) \times \mathbb{R}^{d} \times \mathbb{R}_{d}\right)$, such that $x+\varepsilon \Im g(t, x, \eta) \in \mathscr{N}$, for all $(t, x, \eta) \in K^{\prime}$ and every $\varepsilon \in[0,1]$. Consequently, there exist constants $C, C^{\prime} \in(0, \infty)$ such that, for all $(t, x, \eta) \in K^{\prime}$,

$$
\Im \psi(t, x-\varepsilon \Im g(t, x, \eta), \eta) \geqslant-C|\Im g(t, x-\varepsilon \Im g(t, x, \eta), \eta)|^{3} \geqslant-C^{\prime}|\Im g(t, x, \eta)|^{3} .
$$

Taylor expanding the left hand side with respect to $x$ and using (82) and (84) we obtain

$$
\Im \psi \geqslant \varepsilon|\Im g|^{2}-C^{\prime \prime}\left(|\Im g|^{3}+\varepsilon^{2}|\Im g|^{2}\right)-\varepsilon C_{N_{0}}|\Im \psi|^{N_{0}} \quad \text { on } K^{\prime},
$$

for some $N_{0} \in \mathbb{N}, N_{0} \geqslant 2$, and $C^{\prime \prime}, C_{N_{0}} \in(0, \infty)$. Now, we choose $\varepsilon \in\left(0, \frac{1}{2 C^{\prime \prime}}\right)$ such that $\varepsilon C_{N_{0}}|\Im \psi|^{N_{0}-1}<1 / 2$ on $K^{\prime}$. By possibly restricting our attention to an even smaller real compact neighbourhood, $K^{\prime \prime} \Subset K^{\prime}$, of $\left(t_{0}, x_{0}, \eta_{0}\right)$, we may further ensure that $C^{\prime \prime}|\Im g|<\varepsilon / 4$ on $K^{\prime \prime}$. Then we readily obtain (86) on $K^{\prime \prime}$.

Concerning (87), it only remains to prove the implication " $\Rightarrow$ " because of (75). So, suppose that $(t, x, \eta) \in \mathscr{M}_{\mathbb{R}}$ and $\Im \psi(t, x, \eta)=0$. Then (86) implies that $\Im g(t, x, \eta)=0$ and (77) and (80) show that $(y, \eta):=(k(t, x, \eta), \eta)$ and $(x, g(t, x, \eta))$ are connected by a purely real integral curve of $\widehat{X}_{a}$. Hence, if we had $\Im a(y, \eta)<0$, then we also had $\Im \psi(t, x, \eta)>0$ by (44) and (76)). So, we must have $\Im a(y, \eta)=0$ and Lemma 5.4 implies that $t=0$ or $(t, x, \eta) \in \mathscr{D}$.

(ii) follows from (46), (72), and (81).

To solve the transport equations in the next section we need another consequence of Proposition 5.9 . 
Corollary. 5.11 Locally on $\mathscr{N}$,

$$
k_{(\bar{x}, \bar{\eta})}^{\prime}=\mathcal{O}_{N}\left(\Gamma^{N}\right), \quad g_{(\bar{x}, \bar{\eta})}^{\prime}=\mathcal{O}_{N}\left(\Gamma^{N}\right)
$$

and locally on some small neighbourhood, $\widetilde{N^{\prime}} \subset \widetilde{\mathscr{N}}$, of $\widetilde{\mathscr{E}}$ in $[0, \infty) \times \mathbb{C}^{d} \times \mathbb{R}_{d}$

$$
Q_{(\bar{y}, \bar{\eta})}^{\prime}=\mathcal{O}_{N}\left(\widetilde{\Gamma}^{N}\right), \quad \Xi_{(\bar{y}, \bar{\eta})}^{\prime}=\mathcal{O}_{N}\left(\widetilde{\Gamma}^{N}\right),
$$

PROOF: (91) follows immediatly from (82)-(85). The first equality in (92) is easily derived from (91) by differentiating the identity $k(t, Q(t, y, \eta), \eta)=y$ with respect to $y$ and $(\bar{y}, \bar{\eta})$. To obtain the second we differentiate $g(t, Q(t, y, \eta), \eta)=\Xi(t, y, \eta)$ with respect to $(\bar{y}, \bar{\eta})$ and use the first and again (91).

\section{The transport equations}

In this section we carry out the next step in the construction of a parametrix for the heat operator $h \partial_{t}+\mathrm{Op}_{h}^{W}\left(a_{W}^{\varphi}\right)$. We seek for a parametrix, $\mathcal{Q}$, of the form

$$
\mathcal{Q} f(t, x)=\int e^{i \psi(t, x, \eta) / h-i\langle y \mid \eta\rangle / h} b(t, x, \eta ; h) f(y) \frac{d y d \eta}{(2 \pi h)^{d}},
$$

where $\psi$ is given by (73). For the amplitude $b$ we use the ansatz

$$
b(t, x, \eta ; h) \asymp \sum_{\nu=0}^{\infty} h^{\nu} b_{\nu}(t, x, \eta) .
$$

To find the sequence of transport equations that determines $b_{0}, b_{1}, \ldots$ we ignore for the moment that $\psi$ is defined only in a small neighbourhood of $\mathscr{E}$ and argue formally. (We shall perform everything properly in Proposition 6.3 where the results of this and the previous section are put together.) Observing that

$$
e^{-i \psi / h} \operatorname{Op}_{h}^{W}\left(a_{W}^{\varphi}\right) e^{i \psi / h}=e^{-i \psi / h+\varphi / h} \operatorname{Op}_{h}^{W}\left(i \widetilde{V}-i U_{h}\right) e^{-\varphi / h+i \psi / h}=\operatorname{Op}_{h}^{W}\left(a_{W}^{\varphi-i \psi}\right)
$$

and using Lemma 3.3 with $\phi=\varphi-i \psi$ we see that the equation

$$
\left(h \partial_{t}+\mathrm{Op}_{h}^{W}\left(a_{W}^{\varphi}\right)\right) e^{i \psi / h} b=0
$$

leads to the following sequence of transport equations:

$$
\begin{aligned}
\left(\mathrm{T}_{0}\right) \quad\left(\partial_{t}+Y+\frac{1}{2} \operatorname{div} Y\right) b_{0} & =\mathcal{O}_{N}\left(\Gamma^{N}\right), \quad N \in \mathbb{N}, \\
\left(\mathrm{T}_{1}\right) \quad\left(\partial_{t}+Y+\frac{1}{2} \operatorname{div} Y\right) b_{1} & =P_{2} b_{0}+\mathcal{O}_{N}\left(\Gamma^{N}\right), \quad N \in \mathbb{N}, \\
& \vdots \\
\left(\mathrm{T}_{\nu}\right) \quad\left(\partial_{t}+Y+\frac{1}{2} \operatorname{div} Y\right) b_{\nu} & =\sum_{\varkappa=0}^{\nu-1} P_{\nu+1-\varkappa} b_{\varkappa}+\mathcal{O}_{N}\left(\Gamma^{N}\right), \quad N \in \mathbb{N},
\end{aligned}
$$

Here we have

$$
Y(t, x, \eta)=\left.\sum_{i=1}^{d} \partial_{\xi_{i}} a^{\varphi-i \psi(t, \cdot, \eta)}(x, \xi)\right|_{\xi=0} \partial_{x^{i}}=\sum_{i=1}^{d} \partial_{\xi_{i}} a^{\varphi}\left(x, \psi_{x}^{\prime}(t, x, \eta)\right) \partial_{x^{i}}
$$


and the operators $P_{2}, P_{3}, \ldots$ are given by (33) with $\phi=\varphi-i \psi$. By (74) $\varphi-i \psi-i\langle x \mid \eta\rangle$ depends $(2 \pi \mathbb{Z})^{d}$-periodically on $\eta$ and, hence, the same holds for $Y$ and $P_{\nu}$, so that $\eta$ can be viewed as an element of $\mathbb{T}^{d}$ in the following. As initial conditions we choose

$$
\begin{aligned}
& b_{0}(0, x, \eta):=\chi(x), \\
& b_{\nu}(0, x, \eta):=0, \quad \nu \in \mathbb{N},
\end{aligned}
$$

where $\eta \in \mathbb{T}^{d}$ and $\chi \in C_{0}^{\infty}\left(\mathbb{R}^{d},[0,1]\right)$ is supported in some small neighbourhood of $K_{0}$ and fulfills $\chi \equiv 1$ in another small neighbourhood of $K_{0}$. In the following we also assume that $\chi$ is extended almost analytically such that the extension, $\widetilde{\chi}$, is compactly supported in some tiny complex neighbourhood of $\operatorname{supp}(\chi)$.

Lemma. 6.1 The transport equations $\left(T_{0}\right),\left(T_{1}\right), \ldots$ with initial conditions (96) and (97) are solvable in a small neighbourhood, $\mathscr{N}^{\prime} \subset \mathscr{N}$, of $\mathscr{E}$ in $[0, \infty) \times \mathbb{C}^{d} \times \mathbb{R}_{d}$, which is $2 \pi$-periodic in $\eta$. More generally,

$$
\partial_{(t, x, \bar{x}, \eta)}^{\alpha}\left(\left(\partial_{t}+Y+\frac{1}{2} \operatorname{div} Y\right) b_{\nu}-\sum_{\varkappa=0}^{\nu-1} P_{\nu+1-\varkappa} b_{\varkappa}\right)= \begin{cases}\mathcal{O}_{N, \alpha}\left(\Gamma^{N}\right), & \text { on } \mathscr{N}^{\prime}, \\ \mathcal{O}_{N, \alpha}\left((\Im \psi)^{N}\right), & \text { on } \mathscr{N}^{\prime} \cap \mathscr{M}_{\mathbb{R}},\end{cases}
$$

for every $N \in \mathbb{N}$ and any multiindex $\alpha \in \mathbb{N}_{0}^{3 d+1}$, where the $\mathcal{O}_{N, \alpha}$-symbols are uniform on compact subsets of $\mathscr{N}^{\prime}$. The solutions $b_{\nu}, \nu \in \mathbb{N}_{0}$, are $2 \pi$-periodic in $\eta$. We have $\operatorname{supp}\left(b_{\nu}(t, \cdot, \eta)\right) \subset$ $Q(t, \operatorname{supp}(\widetilde{\chi}), \eta)$, for all $(t, \eta)$ and $\nu \in \mathbb{N}_{0}$.

Proof: The proof is essentially standard; see, e.g., [22, pp. 578]. We have, however, to keep track of the error terms. We argue by induction and consider the equation

$$
\left(\partial_{t}+Z\right) b=f b+w,\left.\quad b\right|_{t=0}=c
$$

where

$$
Z(t, x):=\sum_{i=1}^{d} \partial_{\xi_{i}} a^{\varphi}(x, g(t, x, \eta)) \partial_{x^{i}} .
$$

Moreover, $f:=-\frac{1}{2} \operatorname{div} Y$ and $w$ is either 0 , in which case we set $c=\widetilde{\chi}$, or $w=\sum_{\varkappa=0}^{\nu-1} P_{\nu+1-\varkappa} b_{\varkappa}$, where we suppose that $b_{0}, \ldots, b_{\nu-1}$ have already been constructed. In the latter case $c=0$. We know that

$$
f_{\bar{x}}^{\prime}=\mathcal{O}_{N}\left(\Gamma^{N}\right) \quad \text { on } \mathscr{N}
$$

and as an induction hypothesis we assume that

$$
\left(b_{0}\right)_{\bar{x}}^{\prime}, \ldots,\left(b_{\nu-1}\right)_{\bar{x}}^{\prime}=\mathcal{O}_{N}\left(\Gamma^{N}\right) \quad \text { on } \mathscr{N}^{\prime}:=K^{-1}\left(\widetilde{\mathscr{N}^{\prime}}\right)
$$

which implies

$$
w_{\bar{x}}^{\prime}=\mathcal{O}_{N}\left(\Gamma^{N}\right) \quad \text { on } \mathscr{N}^{\prime} .
$$

Here $\widetilde{N^{\prime}}$ is the neighbourhood of $\mathscr{E}$ appearing in Corollary 5.11 We observe that $Q$ is the flow of $\widehat{Z}:=Z+\bar{Z}$. We first solve the following initial value problem for an ordinary differential equation,

$$
\partial_{t} \tilde{b}(t, y, \eta)=f(t, Q(t, y, \eta), \eta) \tilde{b}(t, y, \eta)+w(t, Q(t, y, \eta), \eta), \quad \tilde{b}(0, y, \eta)=c(y),
$$

and observe that

$$
b(t, x, \eta):=\tilde{b}(t, k(t, x, \eta), \eta)
$$

fulfills

$$
\left(\partial_{t} b+\widehat{Z} b\right)(t, Q(t, y, \eta), \eta)=\frac{d}{d t} b(t, Q(t, y, \eta), \eta)=f(t, Q(t, y, \eta), \eta) \tilde{b}(t, y, \eta)+w(t, Q(t, y, \eta), \eta)
$$


on $\widetilde{\mathbb{N}^{\prime}}$ because of $k(t, Q(t, y, \eta), \eta)=y$. Next, we consider the error term

$$
b_{\bar{x}}^{\prime}(t, x, \eta)=\tilde{b}_{y}(t, k(t, x, \eta), \eta) k_{\bar{x}}^{\prime}(t, x, \eta)+\tilde{b}_{\bar{y}}(t, k(t, x, \eta), \eta) \overline{k_{x}^{\prime}(t, x, \eta)} .
$$

Here the error $k_{\bar{x}}^{\prime}$ can be controlled using (91). To investigate $\tilde{b}_{\bar{y}}$ we differentiate (99) with respect to $\bar{y}$ and obtain

$$
\partial_{t} \tilde{b}_{\bar{y}}^{\prime}(t, y, \eta)-f(t, Q(t, y, \eta), \eta) \tilde{b}_{\bar{y}}^{\prime}(t, y, \eta)=\mathcal{O}_{N}\left(\widetilde{\Gamma}(t, y, \eta)^{N}\right) \quad \text { on } \widetilde{\mathscr{N}}^{\prime}
$$

if we take (92) into account. It also holds $\tilde{b}_{\bar{y}}^{\prime}(0, y, \eta)=c_{\bar{y}}^{\prime}(y)=\mathcal{O}_{N}\left(|\Im y|^{N}\right)$, so we infer from (77) and (102) together with Gronwall's lemma that

$$
\tilde{b}_{\bar{y}}^{\prime}(t, k(t, x, \eta), \eta)=\mathcal{O}_{N}\left(\Gamma(t, x, \eta)^{N}\right) .
$$

In view of (91) we obtain

$$
b_{\bar{x}}^{\prime}(t, x, \eta)=\mathcal{O}_{N}\left(\Gamma(t, x, \eta)^{N}\right), \quad(t, x, \eta) \in \mathscr{N}^{\prime} .
$$

Together with (100) this shows that

$$
\partial_{t} b+Z b=f b+w+\mathcal{O}_{N}\left(\Gamma^{N}\right) \quad \text { on } \mathscr{N}^{\prime} .
$$

Finally, we observe that the components of $Y$ and $Z$ satisfy $Y_{i}-Z_{i}=\mathcal{O}_{N}\left(\Gamma^{N}\right)$, because of (82). The vector field $Z$ may hence be replaced by $Y$ in (103).

Finally, it is clear that $b$ is $2 \pi$-periodic in $\eta$. In fact, the maps $f, w, Q$, and $k$ used to construct $b$ only depend on the equivalence class of $\eta$ in $\mathbb{T}^{d}$. Moreover, it is easy to see that the inclusion supp $(w(t, \cdot, \eta)) \subset Q(t, \operatorname{supp}(\widetilde{\chi}), \eta)$ implies that the support of $b(t, \cdot, \eta)$ is again contained in $Q(t, \operatorname{supp}(\tilde{\chi}), \eta)$. So, we also obtain the last statement of the lemma.

Next, we consider $b_{0}(\tau, x, 0)$ more closely, for $(\tau, x, 0) \in \mathscr{\mathscr { D }}$. By definition of $\mathscr{D}$ we know that there is some $y \in K_{0}$ such that $Q(\tau, y, 0)=x$ and $Q(t, y, 0) \in K_{0}$, for all $t \in[0, \tau]$. By (47), (56), (90), and (95) we further know that, for $t \in[0, \tau]$,

$$
Y(t, Q(t, y, 0), 0)=\nabla_{p} H\left(Q(t, y, 0), \varphi^{\prime}(Q(t, y, 0))\right)=\dot{Q}(t, y, 0) .
$$

In particular, $Q(t, y, 0)$ and $Y(t, Q(t, y, 0), 0)$ are real.

Henceforth we denote the determinant of a real square matrix, $M$, by $|M|$.

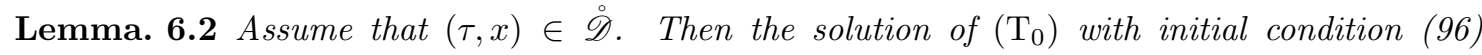
satisfies

$$
b_{0}(\tau, x, 0)=\left|Q_{y}^{\prime}(\tau, y, 0)\right|^{-1 / 2} \chi(y), \quad \text { where } y=\pi_{x} \exp \left(-\tau X_{H}\right)\left(x, \varphi^{\prime}(x)\right) .
$$

Proof: The proof is well-known. We recall it for the sake of completeness. From the proof of Lemma 6.1 and (104) we infer that, for $(t, y, 0) \in \widetilde{\mathscr{D}}$,

$$
\frac{d}{d t} b_{0}(t, Q(t, y, 0), 0)=-\frac{1}{2}\left(b_{0} \operatorname{div} Y\right)(t, Q(t, y, 0), 0) .
$$

The Liouville formula shows that

$$
\frac{d}{d t}\left|Q_{y}^{\prime}(t, y, 0)\right|^{-1 / 2}=-\frac{1}{2}\left|Q_{y}^{\prime}(t, y, 0)\right|^{-1 / 2}(\operatorname{div} Y)(t, Q(t, y, 0), 0)
$$

and it follows that

$$
b_{0}(t, Q(t, y, 0), 0)=\left|Q_{y}^{\prime}(t, y, 0)\right|^{-1 / 2} \chi(y)
$$


In the next proposition we draw the main conclusion of the results attained in Sections 5 and 6 so far.

Up to now the restrictions of the coefficients $b_{\nu}(t, x, \eta), \nu \in \mathbb{N}_{0}$, to the real domain are defined in some $2 \pi$-periodic neighbourhood, $\mathscr{N}^{\prime} \cap \mathscr{M}_{\mathbb{R}}$, of $\mathscr{E}$ which is contained in the domain of $\psi$. In what follows we consider them as functions on $[0, \infty) \times \mathbb{R}^{d} \times \mathbb{T}^{d}$ by simply setting $b_{\nu}$ equal to zero outside $\mathscr{N}^{\prime} \cap \mathscr{M}_{\mathbb{R}}$. We pick some smooth cut-off function, $\varrho \in C_{b}^{\infty}\left([0, \infty) \times \mathbb{R}^{d} \times \mathbb{T}^{d}\right)$, such that $\varrho \equiv 1$ in a neighbourhood of $\mathscr{E}$ and $\operatorname{supp}(\varrho) \subset \mathscr{N}^{\prime} \cap \mathscr{M}_{\mathbb{R}}$, and let $b$ denote a Borel resummation in $S_{b}\left([0, \infty) \times \mathbb{R}^{d} \times \mathbb{T}^{d}\right)$ of the formal series on the right side of

$$
b(t, x, \eta ; h) \asymp \sum_{\nu=0}^{\infty} h^{\nu} b_{\nu}(t, x, \eta) \varrho(t, x, \eta) .
$$

By Proposition 6.1 we then know that there is some $t_{0}>0$ and some compact set $\mathscr{K} \Subset \mathbb{R}^{d}$ such that $\operatorname{supp}(b) \subset\left[0, t_{0}\right] \times \mathscr{K} \times \mathbb{T}^{d}$.

We may assume that we find another compactly supported cut-off function, $\tilde{\varrho} \in C_{0}^{\infty}([0, \infty) \times$ $\left.\mathbb{R}^{d} \times \mathbb{T}^{d},[0,1]\right)$, such that $\tilde{\varrho} \equiv 1$ on $\operatorname{supp}(b)$ and $\operatorname{supp}(\tilde{\varrho}) \subset \mathscr{M}_{\mathbb{R}}$. Extending $\psi$ again by zero outside $\mathscr{M}_{\mathbb{R}}$ we define

$$
\widetilde{\psi}(t, x, \eta):=\tilde{\varrho}(t, x, \eta) \psi(t, x, \eta)+(1-\tilde{\varrho}(t, x, \eta))(\langle x \mid \eta\rangle+i),
$$

for $t \geqslant 0, x \in \mathbb{R}^{d}$, and $\eta \in \mathbb{R}_{d} . \widetilde{\psi}$ is a smooth function such that $\Im \widetilde{\psi}>0$ on $\left([0, \infty) \times \mathbb{R}^{d} \times \mathbb{R}_{d}\right) \backslash \mathscr{E}$ and such that $\widetilde{\psi}(t, x, \eta)-\langle x \mid \eta\rangle$ can be viewed as an element of $S_{b}\left([0, \infty) \times \mathbb{R}^{d} \times \mathbb{T}^{d}\right)$.

Proposition. 6.3 For every $N \in \mathbb{N}$, there is some compactly supported $\check{r}_{N} \in S_{b}\left([0, \infty) \times \mathbb{R}^{d} \times \mathbb{T}^{d}\right)$ with $\operatorname{supp}\left(\check{r}_{N}(t, \cdot, \eta ; h)\right) \subset \mathscr{K}+\{|x| \leqslant R\}$ such that, for sufficiently small $h>0$,

$$
\left(h \partial_{t}+\operatorname{Op}_{h}^{W}\left(a_{W}^{\varphi}\right)\right)\left(e^{i \psi / h} b\right)=h^{N} e^{i \widetilde{\psi} / h} \check{r}_{N} .
$$

Proof: By definition of $a_{W}^{\phi}$ with $\phi=\varphi$ and $\phi=\varphi-i \widetilde{\psi}$, respectively,

$$
e^{-i \widetilde{\psi} / h} \mathrm{Op}_{h}^{W}\left(a_{W}^{\varphi}\right) e^{i \psi / h} b=e^{-i \widetilde{\psi} / h} \mathrm{Op}_{h}^{W}\left(a_{W}^{\varphi}\right) e^{i \widetilde{\psi} / h} b=\mathrm{Op}_{h}^{W}\left(a_{W}^{\varphi-i \widetilde{\psi}}\right) b .
$$

By our choice of $\widetilde{\psi}$ the phase $\varphi-i \widetilde{\psi}+i\langle x \mid \eta\rangle$ defines an element of $S_{b}\left([0, \infty) \times \mathbb{R}^{d} \times \mathbb{T}^{d}\right)$. We may thus apply Lemma 3.3 with $\phi=\varphi-i \widetilde{\psi}$ to the last term in (105) and obtain the following asymptotic expansion in $S_{b}\left([0, \infty) \times \mathbb{R}^{d} \times \mathbb{T}^{d}\right)$,

$$
\begin{aligned}
& e^{-i \widetilde{\psi}(t, x, \eta) / h}\left(\left(h \partial_{t}+\mathrm{Op}_{h}^{W}\left(a^{W}\right)\right) e^{i \psi / h} b\right)(t, x, \eta) \\
& \asymp \quad i\left(\partial_{t} \psi(t, x, \eta)+a^{\varphi}\left(x, \psi_{x}^{\prime}(t, x, \eta)\right)\right) b(t, x, \eta ; h)+h\left(\left(\partial_{t}+Y+\frac{1}{2} \operatorname{div} Y\right) b_{0} \varrho\right)(t, x, \eta) \\
& \quad+\sum_{\nu=2}^{\infty} h^{\nu}\left(\left(\left(\partial_{t}+Y+\frac{1}{2} \operatorname{div} Y\right) b_{\nu-1} \varrho\right)(t, x, \eta)+\sum_{\varkappa=0}^{\nu-2}\left(P_{\nu-\varkappa} b_{\varkappa} \varrho\right)(t, x, \eta)\right),
\end{aligned}
$$

where $Y$ and $P_{\nu}$ are given by (95) and (33) with $\phi=\varphi-i \psi$. (Here we again use that $\psi=\widetilde{\psi}$ on $\operatorname{supp}(b)$.) We observe that

$$
\begin{aligned}
\left(\partial_{t}+Y+\frac{1}{2} \operatorname{div} Y\right)\left(b_{\nu} \varrho\right) & =\left(\left(\partial_{t}+Y+\frac{1}{2} \operatorname{div} Y\right) b_{\nu}\right) \varrho+m_{\nu}, \quad \nu=0,1,2, \ldots \\
P_{\nu-\varkappa}\left(b_{\varkappa} \varrho\right) & =\left(P_{\nu-\varkappa} b_{\varkappa}\right) \varrho+\tilde{m}_{\varkappa, \nu}, \quad 0 \leqslant \varkappa \leqslant \nu, \nu=2,3,4, \ldots,
\end{aligned}
$$

where $m_{\nu}, \widetilde{m}_{\varkappa, \nu} \in C_{0}^{\infty}\left([0, \infty) \times \mathbb{R}^{d} \times \mathbb{T}^{d}\right)$ are supported in supp $\left(\left|\varrho^{\prime}\right|\right) \subset\{\Im \psi>0\}$, which implies

$$
e^{i \widetilde{\psi} / h} m_{\nu}=\mathcal{O}\left(h^{\infty}\right) \quad \text { and } \quad e^{i \widetilde{\psi} / h} \tilde{m}_{\varkappa, \nu}=\mathcal{O}\left(h^{\infty}\right) \quad \text { in } S_{b}\left(\mathbb{R} \times \mathbb{R}^{d} \times \mathbb{T}^{d}\right) .
$$

We next use (89) and the fact that $b_{0}, b_{1}, \ldots$ solve the transport equations $\left(T_{0}\right),\left(T_{1}\right), \ldots$ The error terms in (89) and (98) lead to contributions of order $\mathcal{O}\left(h^{\infty}\right)$ in $S_{b}\left(\mathbb{R} \times \mathbb{R}^{d} \times \mathbb{T}^{d}\right)$, too, because of the elementary estimates

$$
e^{-\Im \psi / h}(\Im \psi)^{N} \leqslant N ! h^{N}, \quad N \in \mathbb{N} .
$$




\section{The construction of $E^{\prime \prime}(0)^{-1}$}

Proposition 6.3 supplies the piece of the parametrix for $E^{\prime \prime}(0)$ corresponding to the set $K_{0} \times(2 \pi \mathbb{Z})^{d}$ as we shall see in the proof of Proposition 7.1 below. Outside $K_{0} \times(2 \pi \mathbb{Z})^{d}$ the Weyl symbol $a_{W}^{\varphi}$ is elliptic and we can use standard methods of pseudodifferential calculus to construct the missing piece of the parametrix. Here are the details:

If $a_{W}^{\varphi}$ were elliptic, we had a well-known asymptotic expansion, $\breve{q}(x, \xi) \asymp \sum_{\nu=0}^{\infty} h^{\nu} q_{\nu}(x, \xi)$, for the Weyl symbol of the inverse operator $\left(\mathrm{Op}_{h}^{W}\left(a_{W}^{\varphi}\right)\right)^{-1}$. We can of course always write down this expansion formally and determine $q_{\nu}$ at all points $(x, \xi)$ where $a_{W}^{\varphi}$ is invertible. We proceed in this way and pick some $\tilde{\chi} \in C_{0}^{\infty}\left(\mathbb{R}^{d},[0,1]\right)$ such that $\widetilde{\chi} \equiv 1$ in a small neighbourhood of $K_{0}$ and $\operatorname{supp}(\tilde{\chi}) \subset\{\chi=1\}$. We recall that $\chi$ has been introduced in (96). Then we define the symbol $\tilde{q} \in S_{b}\left(\mathbb{R}^{d} \times \mathbb{R}_{d}\right)$ to be a Borel resummation of

$$
\sum_{\nu=0}^{\infty} h^{\nu} q_{\nu}(x, \xi)(1-\tilde{\chi}(x))
$$

where now each term is a well-defined function. We let $\#_{h}^{W}$ denote the composition of Weyl symbols, i.e.

$$
\begin{aligned}
& \left(a \#_{h}^{W} c\right)(x, \xi)=\left.e^{i h\left[D_{\eta} D_{x}-D_{y} D_{\xi}\right]} a(y, \eta) c(x, \xi)\right|_{y=x, \eta=\xi} \\
& \quad=\int e^{i\langle\xi-\eta \mid v-x\rangle / h+i\langle\xi-\zeta \mid x-u\rangle / h} a\left(\frac{x+u}{2}, \eta\right) c\left(\frac{x+v}{2}, \zeta\right) \frac{d u d v d \eta d \zeta}{(2 \pi h)^{2 d}},
\end{aligned}
$$

for $a, c \in S_{b}\left(\mathbb{R}^{d} \times \mathbb{R}_{d}\right)$; see, e.g., [12, $\left.\S 2.7\right]$. Then we get, by definition of $q_{\nu}, \nu \in \mathbb{N}_{0}$,

$$
\begin{aligned}
a_{W}^{\varphi} \#_{h}^{W} \tilde{q}(x, \xi) & \asymp \sum_{\alpha, \beta \in \mathbb{N}_{0}^{d}} \frac{h^{|\alpha+\beta|}(-1)^{|\alpha|}}{(2 i)^{|\alpha+\beta|} \alpha ! \beta !}\left(\partial_{x}^{\alpha} \partial_{\xi}^{\beta} a_{W}^{\varphi}(x, \xi)\right)\left(\partial_{\xi}^{\alpha} \partial_{x}^{\beta} \tilde{q}(x, \xi)\right) \\
& =1-\tilde{\chi}(x)+\sum_{\nu=0}^{\infty} h^{\nu} \breve{r}_{\nu}(x, \xi),
\end{aligned}
$$

where each error term $\breve{r}_{\nu}, \nu \in \mathbb{N}_{0}$, contains some partial derivative of $\tilde{\chi}$, which shows that $\operatorname{supp} \breve{r}_{\nu} \subset$ $\{\chi=1\}$, for all $\nu \in \mathbb{N}_{0}$. We now set $q:=\tilde{q} \#_{h}^{W}(1-\chi)$ and infer from (107) that

$$
a_{W}^{\varphi} \#_{h}^{W} q \asymp 1-\chi
$$

in $S_{b}\left(\mathbb{R}^{d} \times \mathbb{R}_{d}\right)$. We observe that the symbol $q$ is again $2 \pi$-periodic in $\xi$. In fact, if we suppose that $a$ and $c$ in (106) are $2 \pi$-periodic in the momentum variables, then we see that $\left(a \#{ }_{h}^{W} c\right)(x, \xi+2 \pi \ell)=$ $\left(a \#{ }_{h}^{W} c\right)(x, \xi), \ell \in \mathbb{Z}^{d}$, by a translation of the variables $\eta$ and $\zeta$ in the oscillatory integral (106). We let $K_{q}(x, y)$ denote the distribution kernel of $\mathrm{Op}_{h}^{W}(q)$. The periodicity of $q$ implies that

$$
K_{q}(x, y)=h^{d} \sum_{z \in \mathbb{Z}_{h}^{d}} \widehat{q}\left(\frac{x+y}{2}, z\right) \delta_{z}(y-x), \quad x, y \in \mathbb{Z}_{h}^{d},
$$

where the Fourier coefficients, $\widehat{q}$, are defined as in (21).

Recalling that $b$ vanishes, for large $t$, we set

$$
I(x, y):=\int_{0}^{\infty} \int_{\mathbb{T}^{d}} e^{i \psi(t, x, \eta) / h-i\langle y \mid \eta\rangle / h} b(t, x, \eta ; h) \frac{d \eta d t}{(2 \pi)^{d} h},
$$

for all $x, y \in \mathbb{Z}_{h}^{d}$, and try the operator $\mathcal{P}$ given by

$$
\mathcal{P}(x, y):=I(x, y)+h^{d} \widehat{q}\left(\frac{x+y}{2}, y-x\right), \quad x, y \in \mathbb{Z}_{h}^{d},
$$

as an approximate inverse for $e^{\varphi / h} E^{\prime \prime}(0) e^{-\varphi / h}$. 
Proposition. 7.1 It holds $\mathcal{P} \in \mathscr{L}\left(\ell^{2}\left(\mathbb{Z}_{h}^{d}\right)\right)$ and

$$
e^{\varphi / h} E^{\prime \prime}(0) e^{-\varphi / h} \mathcal{P}=\mathbb{1}+\mathcal{R},
$$

where $\mathcal{R} \in \mathscr{L}\left(\ell^{2}\left(\mathbb{Z}_{h}^{d}\right)\right)$ such that $\|\mathcal{R}\|=\mathcal{O}_{N}\left(h^{N}\right)$, for every $N \in \mathbb{N}$. In particular,

$$
\left(E^{\prime \prime}(0)\right)^{-1}=e^{-\varphi / h} \mathcal{P}\left(\sum_{m=0}^{\infty}(-1)^{m} \mathcal{R}^{m}\right) e^{\varphi / h} .
$$

Proof: Applying successively Lemma 3.2. Proposition 6.3, and the fact that $b$ vanishes, for large $t$, we get, for all $x, y \in \mathbb{Z}_{h}^{d}$,

$$
\begin{aligned}
& \left(e^{\varphi / h} E^{\prime \prime}(0) e^{-\varphi / h} \circ I\right)(x, y) \\
& =\int_{\mathbb{T}^{d}} \int_{0}^{\infty}\left(e^{\varphi / h} E^{\prime \prime}(0) e^{-\varphi / h} e^{i \psi(t, \cdot, \eta) / h} b(t, \cdot, \eta ; h)\right)(x) e^{-i\langle y \mid \eta\rangle / h} \frac{d t d \eta}{(2 \pi)^{d} h} \\
& =\int_{\mathbb{T}^{d}} \int_{0}^{\infty}\left(\mathrm{Op}_{h}^{W}\left(a_{W}^{\varphi}\right) e^{i \psi(t, \cdot, \eta) / h} b(t, \cdot, \eta ; h)\right)(x) e^{-i\langle y \mid \eta\rangle / h} \frac{d t d \eta}{(2 \pi)^{d} h} \\
& =-\int_{\mathbb{T}^{d}} \int_{0}^{\infty} \partial_{t}\left(e^{i \psi(t, x, \eta) / h-i\langle y \mid \eta\rangle / h} b(t, x, \eta ; h)\right) \frac{d t d \eta}{(2 \pi)^{d}}+\mathcal{R}_{1}(x, y) \\
& =\int_{\mathbb{T}^{d}} e^{i\langle x-y \mid \eta\rangle / h} \chi(x) \frac{d \eta}{(2 \pi)^{d}}+\mathcal{R}_{1}(x, y)=\chi(x) \delta_{x y}+\mathcal{R}_{1}(x, y),
\end{aligned}
$$

where, for $N \in \mathbb{N}$ and $x, y \in \mathbb{Z}_{h}^{d}$,

$$
\mathcal{R}_{1}(x, y):=h^{N} \int_{0}^{\infty} \int_{\mathbb{T}^{d}} e^{i \widetilde{\psi}(t, x, \eta) / h-i\langle y \mid \eta\rangle / h} \check{r}_{N}(t, x, \eta ; h) \frac{d \eta d t}{(2 \pi)^{d} h} .
$$

Since the integrants in (111) vanish, if $t$ is sufficiently large and $\check{r}_{N}(t, \cdot, \eta ; h)$ is supported in $\mathscr{K}+\{|x| \leqslant R\}$, we have

$$
\max \left\{\mathcal{R}_{1}(x, y): \operatorname{dist}(x, \mathscr{K}) \leqslant R, y \in \mathbb{Z}_{h}^{d}\right\}=\mathcal{O}_{N}\left(h^{N}\right), \quad N \in \mathbb{N} .
$$

Moreover, since $\left|\widetilde{\psi}_{\eta}^{\prime}(t, x, \eta)-x\right|$ is uniformly bounded on compact sets, we have $\left|\widetilde{\psi}_{\eta}^{\prime}(t, x, \eta)-y\right| \geqslant$ $|x-y| / 2$ provided $|x-y|$ is sufficiently large. Integrating by parts in (111) by means of the operator

$$
\frac{h}{i} \frac{\left\langle\widetilde{\widetilde{\psi}_{\eta}^{\prime}(t, x, \eta)-y} \mid \nabla_{\eta}\right\rangle}{\mid \widetilde{\psi_{\eta}^{\prime}(t, x, \eta)-\left.y\right|^{2}}}
$$

we therefore find that

$$
\left|\mathcal{R}_{1}(x, y)\right|=\mathcal{O}\left(h^{N}\langle x-y\rangle^{-N}\right), \quad N \in \mathbb{N} .
$$

If we form an operator, $\mathcal{R}_{1}$, whose matrix element at $(x, y) \in \mathbb{Z}_{h}^{d} \times \mathbb{Z}_{h}^{d}$ is given by $\mathcal{R}_{1}(x, y)$, we thus obtain an element in $\mathscr{L}\left(\ell^{1}\left(\mathbb{Z}_{h}^{d}\right)\right) \cap \mathscr{L}\left(\ell^{\infty}\left(\mathbb{Z}_{h}^{d}\right)\right)$. By interpolation we then also have $\mathcal{R}_{1} \in \mathscr{L}\left(\ell^{2}\left(\mathbb{Z}_{h}^{d}\right)\right)$.

Next, we notice that

$$
\mathrm{Op}_{h}^{W}(1-\chi+r)=\mathrm{Op}_{h}^{W}\left(a_{W}^{\varphi}\right) \circ \mathrm{Op}_{h}^{W}(q)=\mathrm{Op}_{h}\left(a_{W}^{\varphi} \# h q\right),
$$

where $r:=a_{W}^{\varphi} \#{ }_{h}^{W} q-(1-\chi)$ is of order $\mathcal{O}\left(h^{\infty}\right)$ in $S_{b}\left(\mathbb{R}^{d} \times \mathbb{R}_{d}\right)$,

$$
a_{W}^{\varphi} \# h q(x, z, \eta):=\int e^{i\langle\xi-\eta \mid x-y\rangle / h} a_{W}^{\varphi}\left(\frac{x+y}{2}, \xi\right) q\left(\frac{y+z}{2}, \eta\right) \frac{d y d \xi}{(2 \pi h)^{d}},
$$


and $\mathrm{Op}_{h}\left(a_{W}^{\varphi} \#_{h} q\right)$ denotes the $h$-pseudodifferential operator defined by the symbol $a_{W}^{\varphi} \#_{h} q \in$ $S_{b}\left(\mathbb{R}^{2 d} \times \mathbb{R}_{d}\right)$. The symbols $r$ and $a_{W}^{\varphi} \#_{h} q$ are both $2 \pi$-periodic in the momentum variable. Moreover,

$$
\left(e^{\varphi / h} E^{\prime \prime}(0) e^{-\varphi / h} \widehat{q}\left(\frac{\cdot+y}{2}, y-\cdot\right)\right)(x)=\left(a_{W}^{\varphi} \#_{h} q\right)^{\wedge}(x, y, y-x),
$$

for $x, y \in \mathbb{Z}_{h}^{d}$, where the Fourier coefficients, $\left(a_{W}^{\varphi} \#_{h} q\right)^{\wedge}(x, y, z), z \in \mathbb{Z}_{h}^{d}$, are given by a formula similar to (21). Since any pseudodifferential operator determines uniquely its distribution kernel, we obtain

$$
\begin{aligned}
& \left(e^{\varphi / h} E^{\prime \prime}(0) e^{-\varphi / h} h^{d} \widehat{q}\left(\frac{+y}{2}, y-\cdot\right)\right)(x) \\
& \quad=\int_{\mathbb{T}^{d}} e^{i\langle\eta \mid x-y\rangle / h}\left(1-\chi\left(\frac{x+y}{2}\right)+r\left(\frac{x+y}{2}, \eta\right)\right) \frac{d \eta}{(2 \pi)^{d}} \\
& \quad=(1-\chi(x)) \delta_{x y}+h^{d} \widehat{r}\left(\frac{x+y}{2}, y-x\right) .
\end{aligned}
$$

Setting

$$
\left(\mathcal{R}_{2} f\right)(x):=h^{d} \sum_{y \in \mathbb{Z}^{d}} \widehat{r}\left(\frac{x+y}{2}, y-x\right) f(y), \quad x \in \mathbb{Z}_{h}^{d}, f \in \ell^{p}\left(\mathbb{Z}_{h}^{d}\right),
$$

where $p \in\{1, \infty\}$, we again have $\mathcal{R}_{2} \in \mathscr{L}\left(\ell^{1}\left(\mathbb{Z}_{h}^{d}\right)\right) \cap \mathscr{L}\left(\ell^{\infty}\left(\mathbb{Z}_{h}^{d}\right)\right)$, because

$$
\left|\widehat{r}\left(\frac{x+y}{2}, y-x\right)\right|=\mathcal{O}\left(h^{N}\langle x-y\rangle^{-N}\right), \quad N \in \mathbb{N},
$$

which is easily verified. By interpolation we see that $\left\|\mathcal{R}_{2}\right\|_{\mathscr{L}\left(\ell^{2}\left(\mathbb{Z}_{h}^{d}\right)\right)}=\mathcal{O}\left(h^{\infty}\right)$. In view of (110) (114) the proof is complete with $\mathcal{R}:=\mathcal{R}_{1}+\mathcal{R}_{2}$.

\section{Calculation of the leading asymptotics}

In this section we calculate the leading asymptotics of $\mathcal{P}\left(x^{\star}, y^{\star}\right)$, where $x^{\star}$ and $y^{\star}$ are our two distinguished points which satisfy Hypothesis 4.4. Integrating by parts repeatedly in the integral defining the Fourier coefficient $\widehat{q}\left(\frac{x^{\star}+y^{\star}}{2}, y^{\star}-x^{\star}\right)$ we see that

$$
\mathcal{P}\left(x^{\star}, y^{\star}\right)=I\left(x^{\star}, y^{\star}\right)+\mathcal{O}\left(h^{\infty}\right)
$$

and it remains to determine the leading asymtotics of $I\left(x^{\star}, y^{\star}\right)$. To this end we recall that $H=$ $T-U$ and that $X_{H}$ denotes the Hamiltonian vector field of $H . \pi: T^{*} \mathbb{R}^{d} \rightarrow \mathbb{R}^{d}$ is the canonical projection in the following. We let $\rho: \mathbb{R} \rightarrow T^{*} \mathbb{R}^{d}$ denote the unique integral curve of $X_{H}$ running in $\mathbb{E}=\{H=0\}$ such that $\gamma:=\pi \rho \uparrow_{[0, \tau]}$ is a minimizing geodesic from $\gamma(0)=y^{\star}$ to $\gamma(\tau)=x^{\star}$, for some suitable $\tau>0$. Recalling the notation introduced in the paragraph preceeding Theorem 2.6 we have

$$
\begin{aligned}
& v_{y^{\star}}=\dot{\gamma}(0)=-\dot{k}\left(\tau, x^{\star}, 0\right), \\
& v_{x^{\star}}=\dot{\gamma}(\tau)=\dot{Q}\left(\tau, y^{\star}, 0\right) .
\end{aligned}
$$

Furthermore, we let $X(t), P(t) \in \mathscr{L}\left(\mathbb{R}^{d}\right), t \in \mathbb{R}$, denote the solution of the initial value problem

$$
\frac{d}{d t}\left(\begin{array}{c}
X(t) \\
P(t)
\end{array}\right)=\left(\begin{array}{cc}
H_{p x}^{\prime \prime}(\rho(t)) & H_{p p}^{\prime \prime}(\rho(t)) \\
-H_{x x}^{\prime \prime}(\rho(t)) & -H_{x p}^{\prime \prime}(\rho(t))
\end{array}\right)\left(\begin{array}{c}
X(t) \\
P(t)
\end{array}\right), \quad\left(\begin{array}{c}
X(0) \\
P(0)
\end{array}\right)=\left(\begin{array}{l}
0 \\
\mathbb{1}
\end{array}\right) .
$$

Proposition. 8.1 The following formula holds, as $h \searrow 0$ :

$$
\begin{aligned}
I\left(x^{\star}, y^{\star}\right) & =\int_{0}^{\infty} \int_{\mathbb{T}^{d}} e^{i \psi\left(t, x^{\star}, \eta\right) / h-i\left\langle y^{\star} \mid \eta\right\rangle / h} b\left(t, x^{\star}, \eta ; h\right) \frac{d \eta d t}{(2 \pi)^{d} h} \\
& =\left(\frac{h}{2 \pi}\right)^{\frac{d-1}{2}}\left|\begin{array}{cc}
0 & -{ }^{t} v_{y^{\star}} \\
v_{x^{\star}} & X(\tau)
\end{array}\right|^{\frac{1}{2}}+\mathcal{O}\left(h^{\frac{d+1}{2}}\right)
\end{aligned}
$$


Proof: We apply the method of complex stationary phase [14, §2] (with respect to $t$ and $\eta$ ) to the integral (119). The critical points of the phase are given by

$$
\begin{aligned}
0 & =\partial_{t} \psi\left(t, x^{\star}, \eta\right), \\
0 & =\nabla_{\eta} \psi\left(t, x^{\star}, \eta\right)-y^{\star} .
\end{aligned}
$$

Of course, a critical point gives a non-vanishing contribution to the asymptotics of (119) only if it is contained in the set $\{\Im \psi=0\}$. By Corollary [5.10 we know that we must have $t=0$ or $\left(t, x^{\star}, \eta\right) \in \mathscr{D}$ in this case. At $t=0$ we have, however, $\nabla_{\eta} \psi\left(0, x^{\star}, \eta\right)=x^{\star} \neq y^{\star}$. So, (90) implies that $(\tau, 0)$ is the only critical point of the phase in (119) where $\varsigma \psi$ vanishes. Again by (90),

$$
\partial_{t}^{2} \psi\left(\tau, x^{\star}, 0\right)=0 .
$$

To study the remaining second derivatives of the phase we differentiate the identity $Q\left(t, k\left(t, x^{\star}, \eta\right), \eta\right)=$ $x^{\star}$ with resect to $t$ and $\eta$ and obtain

$$
\begin{aligned}
Q_{y}^{\prime}\left(t, k\left(t, x^{\star}, \eta\right), \eta\right) \dot{k}\left(t, x^{\star}, \eta\right)+Q_{\bar{y}}^{\prime}\left(t, k\left(t, x^{\star}, \eta\right), \eta\right) \overline{\dot{k}\left(t, x^{\star}, \eta\right)} & =-\dot{Q}\left(t, k\left(t, x^{\star}, \eta\right), \eta\right), \\
Q_{y}^{\prime}\left(t, k\left(t, x^{\star}, \eta\right), \eta\right) k_{\eta}^{\prime}\left(t, x^{\star}, \eta\right)+Q_{\bar{y}}^{\prime}\left(t, k\left(t, x^{\star}, \eta\right), \eta\right) \overline{k_{\eta}^{\prime}\left(t, x^{\star}, \eta\right)} & =-Q_{\eta}^{\prime}\left(t, k\left(t, x^{\star}, \eta\right), \eta\right) .
\end{aligned}
$$

Evaluated at $(t, \eta)=(\tau, 0)$ this yields

$$
\begin{aligned}
Q_{y}^{\prime}\left(\tau, y^{\star}, 0\right) v_{y^{\star}} & =v_{x^{\star}} \\
Q_{y}^{\prime}\left(\tau, y^{\star}, 0\right) \frac{1}{i} \psi_{\eta \eta}^{\prime \prime}(\tau, x, 0) & =i Q_{\eta}^{\prime}\left(\tau, y^{\star}, 0\right),
\end{aligned}
$$

if we take (83), (92), (116), and (117) into account. We consider $i Q_{\eta}^{\prime}\left(\tau, y^{\star}, 0\right)$ more closely in the following. To this end, we recall that the derivative of the flow of $\widehat{X}_{a^{\varphi}}$ along $(\gamma(t), 0), \kappa_{t}^{\prime}\left(y^{\star}, 0\right)$, $t \in[0, \tau]$, is a solution of the Jacobi equation

$$
\frac{d}{d t} \kappa_{t}^{\prime}\left(y^{\star}, 0\right)=\mathbb{F}_{a}(\gamma(t), 0) \kappa_{t}^{\prime}\left(y^{\star}, 0\right)
$$

where $\mathbb{F}_{a}(\gamma(t), 0)$ is given by (69) with $x(t)=\gamma(t)$ and $e=0$. Since we want to determine $i Q_{\eta}^{\prime}\left(\tau, y^{\star}, 0\right)=i \pi_{x}\left(\kappa_{\tau}\right)_{\eta}^{\prime}\left(y^{\star}, 0\right)$, we consider the solution of (124) with initial condition $i\left(\kappa_{0}\right)_{\eta}^{\prime}\left(y^{\star}, 0\right)=$ $(0, i \mathbb{1})$, which we denote by $(\widetilde{X}(t), \widetilde{P}(t))$. It follows that $\Im \widetilde{X} \equiv 0$ and $\Re \widetilde{P} \equiv 0$ and that $(\Re \widetilde{X}, \Im \widetilde{P})$ is a solution of the Jacobi equation

$$
\frac{d}{d t}\left(\begin{array}{l}
\Re \widetilde{X}(t) \\
\Im \widetilde{P}(t)
\end{array}\right)=\left(\begin{array}{cc}
\mathbb{B}(t) & \mathbb{A}(t) \\
0 & -{ }^{t} \mathbb{B}(t)
\end{array}\right)\left(\begin{array}{c}
\Re \widetilde{X}(t) \\
\Im \widetilde{P}(t)
\end{array}\right) .
$$

The matrix in (125) is the fundamental matrix at $(x, p)=(\gamma(t), 0)$ of the Hamiltonian $H_{\varphi}(x, p):=$ $H\left(x, p+\varphi^{\prime}(x)\right)=(H \circ \Xi)(x, p)$, where $\Xi(x, p):=\left(x, p+\varphi^{\prime}(x)\right),(x, p) \in T^{*} \mathbb{R}^{d}$, defines a symplectomorphism. We further recall that $H_{\varphi, x x}^{\prime \prime}(x, 0)=0$, for $x \in K_{0}$, by construction of $\varphi$. Using these remarks we check that $\Re \widetilde{X}=X$, where $(X, P)$ is a solution of (118). In fact, the Jacobi equation (125) is solved by $\left(\Xi^{\prime}\right)^{-1}(\gamma)(X, P)=\left(X,-\varphi^{\prime \prime}(\gamma) X+P\right)$.

In summary, we see that $i Q_{\eta}^{\prime}\left(, \tau, y^{\star}, 0\right)=X(\tau)$. In particular, $i Q_{\eta}^{\prime}\left(, \tau, y^{\star}, 0\right)$ is real and so is $\frac{1}{i} \psi_{\eta \eta}^{\prime \prime}(\tau, x, 0)$ by (123), because $Q_{y}^{\prime}\left(\tau, y^{\star}, 0\right)$ is a real invertible matrix.

By Lemma 6.2 we know that $b_{0}\left(\tau, x^{\star}, 0\right)^{-1}=\left|Q_{y}^{\prime}\left(\tau, y^{\star}, 0\right)\right|^{1 / 2}$. Using also (122) and (123) we thus get

$$
\frac{1}{b_{0}\left(\tau, x^{\star}, 0\right)}\left|\begin{array}{cc}
0 & \frac{1}{i} \partial_{t} \psi_{\eta}^{\prime} \\
\frac{1}{i} \partial_{t} \nabla_{\eta} \psi & \frac{1}{i} \psi_{\eta \eta}^{\prime \prime}
\end{array}\right|^{\frac{1}{2}}=\left|\begin{array}{cc}
1 & 0 \\
0 & \tilde{\kappa}_{\tau}^{\prime}\left(y^{\star}\right)
\end{array}\right|^{\frac{1}{2}}\left|\begin{array}{cc}
0 & -{ }^{t} \dot{\gamma}(0) \\
\dot{\gamma}(0) & \frac{1}{i} \psi_{\eta \eta}^{\prime \prime}
\end{array}\right|^{\frac{1}{2}}=\left|\begin{array}{cc}
0 & -{ }^{t} v_{y^{\star}} \\
v_{x^{\star}} & X(\tau)
\end{array}\right|^{\frac{1}{2}}
$$

where all derivatives of $\psi$ are evaluated at $\left(\tau, x^{\star}, 0\right)$. From Proposition 8.2 below it follows in particular, that the last determinant in the previous equation is non-zero. Therefore, the left 
hand side is non-zero, too. (To show that $\psi_{\eta \eta}^{\prime \prime}\left(\tau, x^{\star}, 0\right)$ is non-singular we could also appeal to the strict positivity of $C_{\tau}$ at $\left(x^{\star}, 0, y^{\star}, 0\right)$.) We may thus split the integral (119) into pieces by means of suitable cut-off functions and apply the stationary (complex) phase formula [14, §2] to a sufficiently small piece of (119) near the critical point $(\tau, 0)$.

Finally, we remark that, for all $\delta, T>0, \inf \left\{\Im \psi\left(t, x^{\star}, \eta\right): t \in[\delta, T], \eta \in \mathbb{T}^{d},|\eta| \geqslant \delta\right\}>0$ and the corresponding piece of the integral (119) gives a contribution of order $\mathcal{O}\left(h^{\infty}\right)$. At $t=0$ we have $\nabla_{\eta}\left(\psi\left(0, x^{\star}, \eta\right)-\left\langle y^{\star} \mid \eta\right\rangle\right)=x^{\star}-y^{\star} \neq 0, \eta \in \mathbb{T}^{d}$, and, for $\eta=0$ and some $\delta^{\prime}, \delta^{\prime \prime}>0$,

$$
\left|\nabla_{\eta}\right|_{\eta=0}\left(\psi\left(t, x^{\star}, \eta\right)-\left\langle y^{\star} \mid \eta\right\rangle\right)|=| k\left(t, x^{\star}, 0\right)-y^{\star} \mid \geqslant \delta^{\prime}, \quad t \in\left[\delta, \tau-\delta^{\prime \prime}\right] \cup\left[\tau+\delta^{\prime \prime}, T\right],
$$

Therefore, the remaining pieces of the integral (119) can be treated by means of integration by parts in $\eta$ and give contributions of order $\mathcal{O}\left(h^{\infty}\right)$, too.

It is of course simple to check that (119) agrees with the already known formula (16) in the translation invariant case. In the next proposition we obtain a generalization of (17).

To prove it we introduce special orthonormal bases with respect to the Finsler structure [5, pp. 31] in the following.

We denote the quadratic form associated with $G\left(z, v_{z}\right)$ by $g_{z}$, for $z=x^{\star}, y^{\star}$, and pick a basis, $\left\langle b_{1}, \ldots, b_{d}\right\rangle$, which is orthonormal with respect to $g_{y^{\star}}$ such that $b_{d}=F\left(y^{\star}, v_{y^{\star}}\right)^{-1} v_{y^{\star}}$. Similarly, we pick another basis, $\left\langle c_{1}, \ldots, c_{d}\right\rangle$, which is orthonormal with respect to $g_{x^{\star}}$ and where $c_{d}=F\left(x^{\star}, v_{x^{\star}}\right)^{-1} v_{x^{\star}}$. We denote the dual basis vectors by $b_{i}^{*}, c_{i}^{*}, 1 \leqslant i \leqslant d$, so that $b_{i}^{*}\left(b_{j}\right)=$ $\sum_{k=1}^{d}\left(b_{i}^{*}\right)_{k} b_{j}^{k}=\delta_{i j}$, etc.

For $1 \leqslant i \leqslant d-1$, we further introduce a Jacobi field, $J_{i}$, which is by definition the solution of the Jacobi equation

$$
D_{\mathscr{T}} D_{\mathscr{T}} J_{i}=R\left(J_{i}, \mathscr{T}\right) \mathscr{T}, \quad J_{i}(0)=b_{i} .
$$

Here $\mathscr{T}$ is the velocity vector field of the unit speed geodesic from $y^{\star}$ to $x^{\star}, D_{\mathscr{T}}$ denotes covariant differentiation in the direction $\mathscr{T}$ with reference vector $\mathscr{T}$, and

$$
R\left(\mathscr{T}, J_{i}\right) \mathscr{T}=\sum_{k, \ell, m, n=1}^{d}\left(\mathscr{T}^{k} R_{k}^{\ell}{ }_{m n} \mathscr{T}^{n}\right) J_{i}^{m} \partial_{x^{\ell}}
$$

where $R_{k}{ }^{\ell} m n$ are the components of the $h h$-curvature tensor of the Chern connection defined by $F$ [5]. Since we only need a well-known Taylor expansion of mutual scalar products of the $J_{i}$ and the formula

$$
\left(\exp _{y}\right)^{\prime}\left(r b_{d}\right) b_{i}=\frac{1}{r} J_{i}(r)
$$

where

$$
r:=d_{F}\left(x^{\star}, y^{\star}\right)
$$

we do not explain these notions further.

Proposition. 8.2 The following identity holds:

$$
\left|\begin{array}{cc}
0 & -{ }^{t} v_{y^{\star}} \\
v_{x^{\star}} & X(\tau)
\end{array}\right|^{\frac{1}{2}}=\frac{1}{\sqrt{\left\langle p_{x^{\star}} \mid v_{x^{\star}}\right\rangle\left\langle p_{y^{\star}} \mid v_{y^{\star}}\right\rangle}} \cdot \frac{\left|G\left(x^{\star}, v_{x^{\star}}\right) G\left(y^{\star}, v_{y^{\star}}\right)\right|^{1 / 4}}{\left|\left(g_{x^{\star}}\left(J_{i}(r), J_{j}(r)\right)\right)_{i, j=1}^{d-1}\right|^{1 / 4}},
$$

where

$$
\left|\left(g_{x^{\star}}\left(J_{i}(r), J_{j}(r)\right)\right)_{i, j=1}^{d-1}\right|^{1 / 4}=d_{F}\left(x^{\star}, y^{\star}\right)^{\frac{d-1}{2}}+\mathcal{O}\left(d_{F}\left(x^{\star}, y^{\star}\right)^{\frac{d}{2}}\right),
$$

as $x^{\star} \rightarrow y^{\star}$. 
Proof: To simplify our notation we denote the distinguished points $x^{\star}$ and $y^{\star}$ simply by $x$ and $y$ in this proof. We let $B$ and $C$ denote the matrices whose $i$-th row is $b_{i}^{*}$ and $c_{i}^{*}$, respectively. From $c_{d}^{*}=F_{v}^{\prime}\left(x, v_{x}\right)=p_{x}$ we infer that $C v_{x}={ }^{t}\left(0 \ldots 0 F\left(x, v_{x}\right)\right)=\left\langle p_{x} \mid v_{x}\right\rangle^{t}(0 \ldots 01)$ and similarly ${ }^{t} v_{y}{ }^{t} B=\left\langle p_{y} \mid v_{y}\right\rangle(0 \ldots 01)$. We thus get

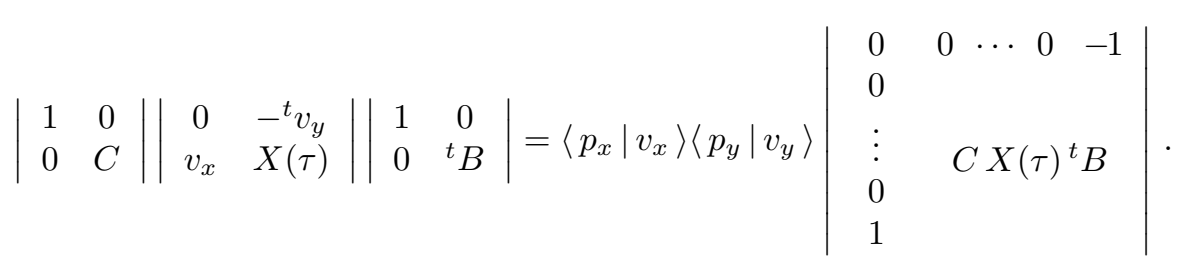

Here the determinant on the right side clearly equals

$$
\operatorname{det}\left(\left(c_{i}^{*} X(\tau)^{t} b_{j}^{*}\right)_{1 \leqslant i, j \leqslant d-1}\right) .
$$

The vectors ${ }^{t} b_{j}^{*}, 1 \leqslant j \leqslant d-1$, are orthogonal to $b_{d} \in \stackrel{\circ}{\mathrm{o}}_{y}$ with respect to the Euclidean scalar product and, hence, span the tangent space of the figuratrix at the momentum which is conjugate to $b_{d}$, i.e. at $p_{y}$. It therefore suffices to consider

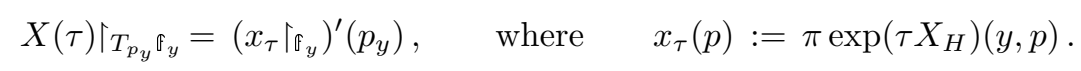

Let $\mathcal{L}: \mathbb{F}_{z} \rightarrow \stackrel{\circ}{\mathrm{a}}_{z}$ denote Legendre transformation, so that $\mathcal{L}$ maps $p \in \mathbb{E}_{z}$ to its conjugate direction $v \in \stackrel{\circ}{z}_{z}$, for $z \in \mathbb{R}^{d}$. It holds $\mathcal{L}\left(p_{x}\right)=c_{d} \| v_{x}$ and $\mathcal{L}\left(p_{y}\right)=b_{d} \| v_{y}$. Moreover, we have [5, p. 410]

$$
\mathcal{L}^{\prime}\left(p_{y}\right)=G\left(y, \mathcal{L}\left(p_{y}\right)\right)^{-1} \uparrow_{T_{p_{y}} \mathbb{F}_{y}}=G\left(y, v_{y}\right)^{-1} \uparrow_{T_{p_{y}} \mathbb{F}_{y}} .
$$

We recall that $G$ is homogenous of degree zero in $v$. Besides we know that

$$
\left(\left(\exp _{y}\right)^{\prime}\left(r \mathcal{L}\left(p_{y}\right)\right) \mathcal{L}\left(p_{y}\right)=\left.\frac{d}{d t}\right|_{t=r} \exp _{y}\left(t \mathcal{L}\left(p_{y}\right)\right) \| v_{x}\right.
$$

where $r=d_{F}(x, y)$. The image of $\mathcal{L}\left(p_{y}\right)$ under $\left(\exp _{y}\right)^{\prime}\left(r \mathcal{L}\left(p_{y}\right)\right)$ is therefore $g_{x}$-orthogonal to $c_{i}$, $1 \leqslant i \leqslant d-1$. (This is precisely the statement of the Gauß lemma in Finsler geometry [5, §6.1.A], as the vectors $c_{i}, 1 \leqslant i \leqslant d-1, \operatorname{span} T_{\mathcal{L}\left(p_{x}\right)^{\triangleright^{\circ}}}$.) In particular,

$$
c_{i}^{*}\left(\exp _{y}\right)^{\prime}\left(r \mathcal{L}\left(p_{y}\right)\right)\left[\mathcal{L}\left(p_{y}\right) \otimes\left(d_{F}\left(x_{\tau}(\cdot), y\right)\right)^{\prime}\right]=0 .
$$

We shall use the following identity,

$$
x_{\tau}(p)=\exp _{y}\left(d_{F}\left(x_{\tau}(p), y\right) \mathcal{L}(p)\right), \quad p \in \mathbb{E}_{y} .
$$

Differentiating (128) and using (126), (127), and $c_{i}^{*}\left(v_{x}\right)=0,1 \leqslant i \leqslant d-1$, we obtain

$$
\begin{aligned}
c_{i}^{*}\left(x_{\tau} \Upsilon_{\mathbb{F}_{y}}\right)^{\prime}\left(p_{y}\right)^{t} b_{j}^{*} & =r c_{i}^{*}\left(\left(\exp _{y}\right)^{\prime}\left(r \mathcal{L}\left(p_{y}\right)\right) G\left(y, v_{y}\right)^{-1 t} b_{j}^{*}\right. \\
& =r c_{i}^{*}\left(\left(\exp _{y}\right)^{\prime}\left(r b_{d}\right)\right) b_{j},
\end{aligned}
$$

for $1 \leqslant i, j \leqslant d-1$. Let $M$ be the matrix with entries $c_{i}^{*}\left(\left(\exp _{y}\right)^{\prime}\left(r b_{d}\right)\right) b_{j}, 1 \leqslant i, j \leqslant d-1$. It holds $\sum_{i=1}^{d-1} t c_{i}^{*} c_{i}^{*}=G\left(x, v_{x}\right)\left\lceil_{T_{\mathcal{L}\left(p_{x}\right)^{\circledR}{ }^{\circ}}}\right.$, whence we get

$$
\begin{aligned}
\left({ }^{t} M M\right)_{i j} & =g_{x}\left(\left(\exp _{y}\right)^{\prime}\left(r b_{d}\right) b_{i},\left(\exp _{y}\right)^{\prime}\left(r b_{d}\right) b_{j}\right) \\
& =\frac{1}{r^{2}} g_{x}\left(J_{i}(r), J_{j}(r)\right) \\
& =\delta_{i j}-\frac{r^{2}}{3} g_{y}\left(R\left(b_{i}, b_{d}\right) b_{d}, b_{j}\right)+\mathcal{O}\left(r^{3}\right) .
\end{aligned}
$$

Here the well-known Taylor expansion in the last line is derived using the formulas of [5, §5.5]. We conclude the proof of the proposition by noticing that $(\operatorname{det} B)^{2}=\operatorname{det}\left({ }^{t} B B\right)=\operatorname{det} G\left(y, v_{y}\right)$ and $\operatorname{det} C=\left(\operatorname{det} G\left(x, v_{x}\right)\right)^{1 / 2}$. 


\section{A Applicability of a result by Bach and Møller}

In this appendix we show that we can apply the results of 4 in our situation and thus obtain a proof of Theorem 2.4. The starting point will be a Helffer-Sjöstrand formula established in [13. It involves the inverse of a certain operator which is introduced in the following. First, we recall that we have fixed some pure, tempered Gibbs measure, $\mu_{\beta, h}$, and have set $\mathscr{H}^{0}=L^{2}\left(\mu_{\beta, h}\right)$. We further set $\mathscr{H}^{1}:=L^{2}\left(\mu_{\beta, h}\right) \widehat{\otimes} \ell^{2}\left(\mathbb{Z}_{h}^{d}\right)$, where $\widehat{\otimes}$ deontes the (completed) tensor product of Hilbert spaces, and

$$
\begin{aligned}
& \Omega^{0}:=\left\{f: \mathbb{R}^{\mathbb{Z}_{h}^{d}} \rightarrow \mathbb{C}: \exists n \in \mathbb{N}, x_{1}, \ldots, x_{n} \in \mathbb{Z}_{h}^{d}, f_{n} \in C_{b}^{\infty}\left(\mathbb{R}^{n}\right)\right. \\
& \left.\forall \sigma \in \mathbb{R}^{\mathbb{Z}_{h}^{d}}: f(\sigma)=f_{n}\left(\sigma_{x_{1}}, \ldots, \sigma_{x_{n}}\right)\right\} .
\end{aligned}
$$

Then $\Omega^{0}$ is a dense subspace of $\mathscr{H}^{0}$ and the algebraic tensor product $\Omega^{1}:=\Omega^{0} \otimes\left\langle e_{x}: x \in \mathbb{Z}_{h}^{d}\right\rangle$ is a dense subspace of $\mathscr{H}^{1}$. Here $\left\langle e_{x}: x \in \mathbb{Z}_{h}^{d}\right\rangle$ is the linear hull of the canonical orthonormal basis of $\ell^{2}\left(\mathbb{Z}_{h}^{d}\right)$. We next define two operators, $\mathscr{L}_{\beta, h}^{(i)}, i=1,2$, on $\mathscr{H}^{i}$ with $\operatorname{domain} \operatorname{dom}\left(\mathscr{L}_{\beta, h}^{(i)}\right):=\Omega^{i}$ by

$$
\mathscr{L}_{\beta, h}^{(0)} f(\sigma):=\sum_{x \in \mathbb{Z}_{h}^{d}}\left(-\beta^{-1} \partial_{\sigma_{x}}^{2} f(\sigma)+\partial_{\sigma_{x}} E_{\{x\}}\left(\sigma_{x} \mid \sigma_{\{x\}^{c}}\right) \partial_{\sigma_{x}} f(\sigma)\right),
$$

for $\sigma \in \mathbb{R}^{\mathbb{Z}_{h}^{d}}$ and $f \in \Omega^{0}$, and

$$
\mathscr{L}_{\beta, h}^{(1)}:=\mathscr{L}_{\beta, h}^{(0)} \otimes \mathbb{1}+E^{\prime \prime} .
$$

We recall that the matrix element of $E^{\prime \prime}$ at $\sigma \in \mathbb{R}_{h}^{d}$ is defined by (11). Then $\mathscr{L}_{\beta, h}^{(i)}$ is essentially selfadjoint on $\mathscr{H}^{i}$, for $i=0,1$ [13]. We denote its unique selfadjoint extension by $L_{\beta, h}^{(i)}$. We recall a result from [13. which permits to locate spectral gaps at the bottom of the spectrum of $L_{\beta, h}^{(i)}$ at large inverse temperatures. To this end, we set

$$
\begin{aligned}
& m_{\star}:=\inf _{x \in \mathbb{R}^{d}} D_{\theta \theta}^{\prime \prime}(x, 0)-J \sum_{|\ell| \leqslant R} \sup _{x \in \mathbb{R}^{d}} W_{\theta \theta}^{\prime \prime}(x, \ell, 0), \\
& m^{\star}:=\sup _{x \in \mathbb{R}^{d}} D_{\theta \theta}^{\prime \prime}(x, 0)+2 J \sum_{|\ell| \leqslant R} \sup _{x \in \mathbb{R}^{d}} W_{\theta \theta}^{\prime \prime}(x, \ell, 0),
\end{aligned}
$$

and assume that there is some $n \in \mathbb{N}$ such that

$$
(n+1) m_{\star}-n m^{\star}>0 .
$$

Note that this is always true, for fixed $n$, provided $J>0$ is sufficiently small. Moreover, we set, for $\varepsilon>0$,

$$
\mathcal{I}(n, \varepsilon):=\bigcup_{\nu=1}^{n}\left(\nu m_{\star}-\varepsilon, \nu m^{\star}+\varepsilon\right)
$$

Theorem. A.1 For every $\varepsilon>0$, there is some $\beta_{0} \geqslant 1$ such that, for all $\beta \geqslant \beta_{0}$ and $h \in(0,1]$,

$$
\begin{aligned}
\operatorname{spec}\left(L_{\beta, h}^{(0)}\right) \cap\left(-\infty,(n+1) m_{\star}-\varepsilon\right] & \subset \mathcal{I}(n, \varepsilon) \cup\{0\}, \\
\operatorname{spec}\left(L_{\beta, h}^{(1)}\right) \cap\left(-\infty,(n+1) m_{\star}-\varepsilon\right] & \subset \mathcal{I}(n, \varepsilon) .
\end{aligned}
$$

Next, we introduce an effective Hamiltonian, or, Feshbach operator, $\mathcal{F}_{\beta, h}$, associated with $L_{\beta, h}^{(1)}$. To this end we denote the projection on $\mathscr{H}^{0}$ onto the subspace of constant functions by $\mathrm{p}$, and set $P:=\mathrm{p} \otimes \mathbb{1}$. We write $\mathrm{p}^{\perp}=\mathbb{1}-\mathrm{p}$ and $P^{\perp}=\mathrm{p}^{\perp} \otimes \mathbb{1}$. In view of Theorem A.1 $\mathcal{F}_{\beta, h}$ is then well-defined by

$$
\begin{aligned}
\mathcal{F}_{\beta, h} & :=P L_{\beta, h}^{(1)} P-P L_{\beta, h}^{(1)} P^{\perp}\left(P^{\perp} L_{\beta, h}^{(1)} P^{\perp}\right)^{-1} P^{\perp} L_{\beta, h}^{(1)} P \\
& =P E^{\prime \prime} P-P E^{\prime \prime} P^{\perp}\left(P^{\perp} L_{\beta, h}^{(1)} P^{\perp}\right)^{-1} P^{\perp} E^{\prime \prime} P
\end{aligned}
$$


Now we are in a position to write down the Helffer-Sjöstrand formula for our lattice spin model. Its validity is also proved in [13]. We note that it only holds if we assume $\mu_{\beta, h}$ to be pure. The crucial point is that $\operatorname{ker} L_{\beta . h}^{(0)}=\mathbb{C} 1$ if and only if $\mu_{\beta, h}$ is pure.

Theorem. A.2 There is some $\beta_{0} \geqslant 1$ such that, for all $\beta \geqslant \beta_{0}, h \in(0,1]$, and $x, y \in \mathbb{Z}_{h}^{d}$,

$$
\operatorname{Cor}_{\beta, h}\left(\sigma_{x} ; \sigma_{y}\right)=\frac{1}{\beta}\left\langle e_{x} \mid\left(L_{\beta, h}^{(1)}\right)^{-1} e_{y}\right\rangle_{\mathscr{H}^{1}}=\frac{1}{\beta}\left\langle e_{x} \mid\left(\mathcal{F}_{\beta, h}\right)^{-1} e_{y}\right\rangle_{\mathscr{H}^{1}},
$$

where $e_{z} \equiv 1 \otimes e_{z}$, for $z \in \mathbb{Z}_{h}^{d}$.

To obtain a proof of Theorem 2.4 we only have to verify certain conditions that allow to apply [4. Theorems $3.3 \& 3.7$ ] to our situation. In fact, it suffices to derive a certain estimate involving the operators $L_{\beta, h}^{(i)}$ which is done in the next lemma. The main idea behind all this is that, due to the small temperature localization at the global minimum 0 of the spin system, we expect the Feshbach operator $\mathcal{F}_{\beta, h}$ to be well-approximated by $E^{\prime \prime}(0)$; see (130). Before we come to that we have, however, to introduce some further notation.

We let $\mathrm{D}_{E} \in \mathscr{L}\left(\ell^{2}\left(\mathbb{Z}_{h}^{d}\right)\right)$ denote the diagonal part of $E^{\prime \prime}(0)$, that is, $\mathrm{D}_{E} e_{x}:=E^{\prime \prime}(0)_{x x} e_{x}$, for $x \in \mathbb{Z}_{h}^{d}$. Furthermore, we set

$$
\begin{aligned}
\mathrm{T} & :=\mathrm{D}_{E}^{-1 / 2}\left(\mathrm{D}_{E}-J^{-1} E^{\prime \prime}(0)\right) \mathrm{D}_{E}^{-1 / 2}, \\
\mathrm{~V} & :=\left(\mathbb{1} \otimes \mathrm{D}_{E}^{-1 / 2}\right)\left(E^{\prime \prime}-\mathbb{1} \otimes E^{\prime \prime}(0)\right)\left(\mathbb{1} \otimes \mathrm{D}_{E}^{-1 / 2}\right), \\
\mathrm{B}_{0} & :=L_{\beta}^{(0)} \otimes \mathrm{D}_{E}^{-1}+\mathbb{1}, \\
\mathrm{B} & :=L_{\beta}^{(0)} \otimes \mathrm{D}_{E}^{-1}+\mathbb{1} \otimes\left(\mathrm{D}_{E}^{-1 / 2} E^{\prime \prime}(0) \mathrm{D}_{E}^{-1 / 2}\right)=\mathrm{B}_{0}-J \mathbb{1} \otimes \mathrm{T} .
\end{aligned}
$$

Identifying $\ell^{2}\left(\mathbb{Z}_{h}^{d}\right)$ with $\operatorname{Ran} P=\mathbb{C} 1 \otimes \ell^{2}\left(\mathbb{Z}_{h}^{d}\right)$ we have

$$
\left(\mathbb{1} \otimes \mathrm{D}_{E}^{-1 / 2}\right) \mathcal{F}_{\beta, h}\left(\mathbb{1} \otimes \mathrm{D}_{E}^{-1 / 2}\right)=\mathbb{1}-J \mathrm{~T}+\beta^{-1 / 2} \mathrm{Y},
$$

where

$$
\mathrm{Y}:=\beta^{1 / 2} P \vee P-\beta^{1 / 2} P \vee P^{\perp}\left(P^{\perp}(\mathrm{B}+\mathrm{V}) P^{\perp}\right)^{-1} P^{\perp} \mathrm{V} P .
$$

Lemma. A.3 There exist $\beta_{0} \geqslant 1, J_{0}>0$, and $\vartheta \in(0,1)$ such that, for all $\beta \geqslant \beta_{0}, J \in\left(0, J_{0}\right]$, and $x, y \in \mathbb{Z}_{h}^{d}$,

$$
\left|\mathrm{Y}_{x y}\right| \leqslant\left\{(\mathbb{1}-\vartheta J \mathrm{~T})^{-1}\right\}_{x y}
$$

Proof: Combining the estimate succeeding Equation (53) of [13] and Lemma 7.4 of [13] we see that there is some constant $C \in(0, \infty)$ such that, for all sufficiently small $J>0$ and all $x, y \in \mathbb{Z}_{h}^{d}$,

$$
\left(E_{x x}^{\prime \prime}(0) E_{y y}^{\prime \prime}(0)\right)^{-1 / 2}\left|E_{x y}^{\prime \prime}-E_{x y}^{\prime \prime}(0)\right| \leqslant \frac{C}{\beta^{1 / 2}}\left(\delta_{x y}+J \mathrm{~T}_{x y}\right)\left(L_{\beta}^{(0)}+1\right)
$$

in the sense of quadratic forms on $\Omega^{0}$. Here we also use that we have a strictly positive uniform lower bound for $E_{x x}^{\prime \prime}(0), x \in \mathbb{Z}_{h}^{d}$, provided $J>0$ is sufficiently small. Moreover, we use that there is some $c>0$ such that, for all $x, y \in \mathbb{Z}_{h}^{d}$, we have the bound $\widetilde{J}_{x y} \leqslant c J \mathrm{~T}_{x y}$, where the $\widetilde{J}_{x y} \geqslant 0$ are the constants appearing in [13, §7]. The form bound (132) clearly implies that

$$
\left|\left\langle e_{x} \mid P \vee P e_{y}\right\rangle_{\mathscr{H}^{1}}\right|=\frac{\left|\left\langle 1 \mid\left(E_{x y}^{\prime \prime}-E_{x y}^{\prime \prime}(0)\right) 1\right\rangle_{\mathscr{H}}\right|}{\sqrt{E_{x x}^{\prime \prime}(0) E_{y y}^{\prime \prime}(0)}} \leqslant \frac{C}{\beta^{1 / 2}}\left(\delta_{x y}+J \mathrm{~T}_{x y}\right),
$$

because $L_{\beta}^{(0)} 1=0$. Since

$$
\delta_{x y}+J \mathrm{~T}_{x y}<\left\{\sum_{n=0}^{\infty}(J \mathrm{~T})^{n}\right\}_{x y}=\left\{(\mathbb{1}-J \mathrm{~T})^{-1}\right\}_{x y},
$$


due to the fact that $\mathbf{T}_{x y} \geqslant 0$ with strict inequality at least for $|x-y| / h=1$, there is some $\tilde{\vartheta} \in(0,1)$ such that

$$
\beta^{1 / 2}\left|\left\langle e_{x} \mid P \vee P e_{y}\right\rangle_{\mathscr{H}}\right| \leqslant C\left\{(\mathbb{1}-\tilde{\vartheta} J \mathrm{~T})^{-1}\right\}_{x y} .
$$

We set $\overline{\mathrm{B}}_{0}:=P^{\perp} \mathrm{B}_{0} P^{\perp}, \overline{\mathrm{B}}:=P^{\perp} \mathrm{B} P^{\perp}$, and $\overline{\mathrm{V}}:=P^{\perp} \mathrm{V} P^{\perp}$ in the following. Since the numbers $E_{x x}^{\prime \prime}(0)$ are bounded from above uniformly in $x \in \mathbb{Z}_{h}^{d}$, it also follows from (132) that there is some $\widetilde{C} \in(0, \infty)$ such that

$$
\left\|\left\{\mathrm{B}_{0}^{-1 / 2} \mathrm{VB}_{0}^{-1 / 2}\right\}_{x y}\right\|+\left\|\left\{\overline{\mathrm{B}}_{0}^{-1 / 2} \overline{\mathrm{V}} \overline{\mathrm{B}}_{0}^{-1 / 2}\right\}_{x y}\right\| \leqslant \frac{\widetilde{C}}{\beta^{1 / 2}}\left(\delta_{x y}+J \mathrm{~T}_{x y}\right)
$$

for all $x, y \in \mathbb{Z}_{h}^{d}$. Now let $\varepsilon \in\left(0, m^{\star}-m_{\star}\right)$. By Theorem 2.4(i) we know that, for sufficiently large $\beta \geqslant 1$,

$$
\overline{\mathrm{B}}_{0} \geqslant \frac{m_{\star}-\varepsilon}{m^{\star}}+1=: \frac{1}{\vartheta^{\prime}},
$$

where $\vartheta^{\prime} \in(0,1)$. This implies, for all $x, y \in \mathbb{Z}_{h}^{d}$,

$$
\left\|\left\{\overline{\mathrm{B}}_{0}^{-1 / 2} J \mathrm{\top} \overline{\mathrm{B}}_{0}^{-1 / 2}\right\}_{x y}\right\| \leqslant J \mathrm{~T}_{x y}\left\|\overline{\mathrm{B}}_{0}^{-1}\right\| \leqslant \vartheta^{\prime} J \mathrm{~T}_{x y},
$$

which permits to get

$$
\left\|\left\{\overline{\mathrm{B}}_{0}^{1 / 2} \overline{\mathrm{B}}^{-1} \overline{\mathrm{B}}_{0}^{1 / 2}\right\}_{x y}\right\| \leqslant\left\|\left\{\sum_{n=0}^{\infty}\left(\overline{\mathrm{B}}_{0}^{-1 / 2} J \mathrm{~T} \overline{\mathrm{B}}_{0}^{-1 / 2}\right)^{n}\right\}_{x y}\right\| \leqslant\left\{\left(\mathbb{1}-\vartheta^{\prime} J \mathrm{~T}\right)^{-1}\right\}_{x y} .
$$

Using (134) and (135) we can now copy the proof of [4, Theorem 4.6] to get

$$
\beta^{1 / 2}\left|\left\langle e_{x} \mid P \vee P^{\perp}\left(P^{\perp}(\mathrm{B}+\mathrm{V}) P^{\perp}\right)^{-1} P^{\perp} \bigvee P e_{y}\right\rangle\right| \leqslant C^{\prime}\left\{\left(\mathbb{1}-\vartheta^{\prime} J \mathrm{~T}\right)^{-1}\right\}_{x y},
$$

for some $C^{\prime} \in(0, \infty)$. The previous estimate together with (133) implies the assertion of the lemma.

The proof of Theorem 2.4(iii) now follows from Lemma A.3 together with [4, Theorems $3.3 \&$ \& 3.7].

\section{B Some elementary linear algebra}

In this appendix we prove that Formula (16) of Theorem 2.5 yields Formula (17).

We let $N: \mathbb{f} \rightarrow S^{d-1}$ denote the exterior normal field on $\mathbb{f}$. (Note that, of course, the figuratrix does not depend on $x$ in the translation invariant case.) The derivative of its inverse is given by $\left(N^{-1}\right)^{\prime}(\stackrel{v}{v})=\left|H_{p}^{\prime}(p(\grave{v}))\right|\left(H_{p p}^{\prime \prime}(p(\grave{v}))^{\perp}\right)^{-1}$, for $\dot{v} \in S^{d-1}$. On the other hand we have $F(v)=\left\langle v \mid N^{-1}(\dot{v})\right\rangle$ and direct calculations show that the Hessian of $F$ at $v$ restricted to the

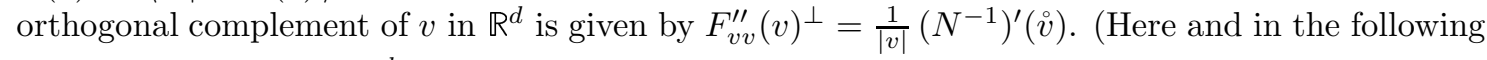
we always assume $v \in \mathbb{R}^{d} \backslash\{0\}$ and set $\stackrel{0}{v}=v /|v|$.) This implies

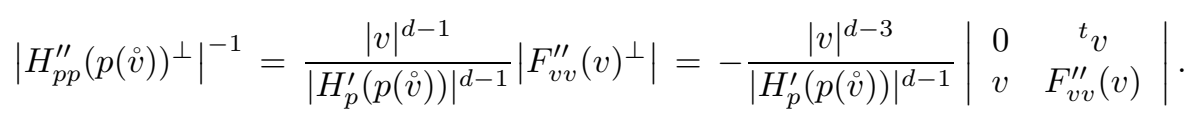

Next, we pick a basis, $\left\langle b_{1}, \ldots, b_{d}\right\rangle$, which is orthonormal with respect to $G(v)=\frac{1}{2}\left(F^{2}\right)_{v^{i} v^{j}}^{\prime \prime}(v)$ and such that $b_{d}=F(v)^{-1} v$. We denote its dual basis by $\left\langle b_{1}^{*}, \ldots, b_{d}^{*}\right\rangle$. Furthermore, we denote the matrix whose $i$-th row is $b_{i}^{*}$ by $B$ and the matrix whose $i$-th column is $b_{i}$ by $Q$, so that $B Q=\mathbb{1}_{d}$. Then [5, §2.2]

$$
F_{v v}^{\prime \prime}(v)={ }^{t} B\left(\begin{array}{cc}
\frac{1}{F(v)} \mathbb{1}_{d-1} & 0 \\
0 & 0
\end{array}\right) B, \quad|Q|^{-2}=|G(v)|, \quad\left({ }^{t} Q v\right)_{d}=\frac{|v|^{2}}{F(v)} .
$$


Consequently, we get

$$
\left|\begin{array}{cc}
0 & { }^{t} v \\
v & F_{v v}^{\prime \prime}(v)
\end{array}\right|=|Q|^{-2}\left|\begin{array}{ccc}
0 & { }^{t} v Q & \\
{ }^{t} Q v & \frac{1}{F(v)} \mathbb{1}_{d-1} & 0 \\
0 & 0 & 0
\end{array}\right|=-|G(v)| \frac{|v|^{4}}{F(v)^{d+1}} .
$$

From (136), (137) and the homogenity of $F$ we infer that

$$
\frac{\left|H_{p}^{\prime}(p(v))\right|^{d-3}}{\left|H_{p p}^{\prime \prime}(p(v))^{\perp}\right||v|^{d-1}}=\frac{1}{\left|H_{p}^{\prime}(p(v))\right|^{2} F(\stackrel{\circ}{*})^{2}} \frac{|G(v)|}{F(v)^{d-1}} .
$$

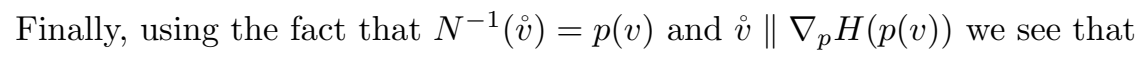

$$
\left|H_{p}^{\prime}(p(v))\right|^{2} F\left(\stackrel{\circ}{v^{2}}=\left\langle\nabla_{p} H(p(v)) \mid p(v)\right\rangle^{2} .\right.
$$

This shows that (16) and (17) are equivalent.

\section{Basic facts about almost analytic extensions}

We recall some notions developed in [14]: $\Omega$ always denotes some open subset of $\mathbb{C}^{n}$ in the following and $\Omega_{\mathbb{R}}:=\Omega \cap \mathbb{R}^{n}$. Two functions, $g_{1}, g_{2} \in C^{\infty}(\Omega, \mathbb{C})$, are called equivalent, in symbols $g_{1} \sim g_{2}$, iff, for every compact $\Omega^{\prime} \Subset \Omega$ and $N \in \mathbb{N}$, there exists a constant $C_{N, \Omega^{\prime}} \in(0, \infty)$ such that

$$
\left|g_{1}(z)-g_{2}(z)\right| \leqslant C_{N, \Omega^{\prime}}|\Im z|^{N}, \quad z \in \Omega^{\prime} .
$$

We recall a technical lemma from [14] which is used very often in the main text.

Lemma. C.1 Let $\mathcal{G} \subset \mathbb{R}^{n}$ be open, $f \in C_{b}^{\infty}(\mathcal{G})$, and $g: \mathcal{G} \rightarrow \mathbb{R}$ be locally Lipschitz continuous, i.e. for all compact $\mathcal{G}^{\prime} \Subset \mathcal{G}$ there is some $C_{\mathcal{G}^{\prime}} \in(0, \infty)$ such that $|g(x)-g(y)| \leqslant C_{\mathcal{G}^{\prime}}|x-y|$, $x, y \in \mathcal{G}^{\prime}$. Assume that $\operatorname{supp}(g)=\mathcal{G}$ and that, for all compact $\mathcal{G}^{\prime} \Subset \mathcal{G}$ and $N \in \mathbb{N}$, there is some $C_{N, \mathcal{G}^{\prime}} \in(0, \infty)$ such that

$$
|f(x)| \leqslant C_{N, \mathcal{G}^{\prime}}|g(x)|^{N}, \quad x \in \mathcal{G}^{\prime} .
$$

Then, for all compact $\mathcal{G}^{\prime} \Subset \mathcal{G}, N \in \mathbb{N}$, and $\alpha \in \mathbb{N}_{0}^{n},|\alpha|<N$, there exists some $C_{N, \mathcal{G}^{\prime}, \alpha} \in(0, \infty)$ such that

$$
\left|\partial_{x}^{\alpha} f(x)\right| \leqslant C_{N, \mathcal{G}^{\prime}, \alpha}|g(x)|^{N-|\alpha|}, \quad x \in \mathcal{G}^{\prime} .
$$

Let $g_{1}, g_{2} \in C^{\infty}(\Omega, \mathbb{C})$ be equivalent. By the previous lemma we find, for every compact $\Omega^{\prime} \Subset \Omega$, $N \in \mathbb{N}$, and all multi-indices $\alpha, \beta \in \mathbb{N}_{0}^{n}$, some $C_{N, \Omega^{\prime}, \alpha, \beta} \in(0, \infty)$ such that

$$
\left|\partial_{\Re z}^{\alpha} \partial_{\Im z}^{\beta}\left(g_{1}(z)-g_{2}(z)\right)\right| \leqslant C_{N, \Omega^{\prime}, \alpha, \beta}|\Im z|^{N}, \quad z \in \Omega^{\prime} .
$$

A function $\tilde{f} \in C^{\infty}(\Omega, \mathbb{C})$ is called almost analytic, iff $\partial_{\bar{z}_{i}} \tilde{f} \sim 0$, for $i=1, \ldots, n$. We denote the set of all almost analytic functions on $\Omega$ by $C^{\text {aa }}(\Omega, \mathbb{C})$. Furthermore, let $f \in C^{\infty}\left(\Omega_{\mathbb{R}}, \mathbb{C}\right)$. Then $\tilde{f} \in C^{\infty}(\Omega, \mathbb{C})$ is called an almost analytic extension of $f$, iff $\tilde{f} \in C^{\text {aa }}(\Omega, \mathbb{C})$ and $\tilde{f} \uparrow_{\Omega_{\mathbb{R}}}=f$. We denote the set of all almost analytic extensions of $f$ by $C^{\text {aa }}[f]$. For instance, every $f \in C_{b}^{\infty}\left(\mathbb{R}^{n}, \mathbb{C}\right)$ possesses almost analytic extensions and $\tilde{f}_{1}, \tilde{f}_{2} \in C^{\text {aa }}[f]$ fulfill $f_{1} \sim f_{2}$.

Conversely, if $\tilde{f} \in C^{\text {aa }}[f]$ and $g \sim 0$, then again $\tilde{f}+g \in C^{\text {aa }}[f]$, so that $C^{\text {aa }}[f]$ is an equivalence class modulo $\sim$. For every $\delta>0$, there is some $\tilde{f} \in C^{\text {aa }}[f]$ with support contained in $\{z \in$ $\Omega:|\Im z| \leqslant \delta\}$. Moreover, for $\tilde{f} \in C^{\text {aa }}[f]$, for all multi-indices $\alpha, \beta \in \mathbb{N}_{0}^{n}$, and $i=1, \ldots, n$, it holds $\partial_{\Re z}^{\alpha} \partial_{\Im z}^{\beta} \partial_{\bar{z}_{i}} \tilde{f} \sim 0$ by Lemma C.1. In particular, it follows that any partial derivative of $\tilde{f} \in C^{\text {aa }}(\Omega, \mathbb{C})$ is again almost analytic.

Acknowledgement. I am gratefully indepted to Johannes Sjöstrand for proposing to study the problems addressed in this article and for many helpful discussions. This work was supported by the IHP network HPRN-CT-2002-00277 from the European Union. 


\section{References}

[1] S. Albeverio, Yu. G. Kondratiev, M. Röckner, and T. V. Tsikalenko. A priori estimates for symmetrizing measures and their applications to Gibbs states. J. Funct. Anal., 171: 366-400, 2000 .

[2] V. Bach, T. Jecko, and J. Sjöstrand. Correlation asymptotics of classical lattice spin systems with nonconvex Hamilton function at low temperature. Ann. Henri Poincaré, 1: 59-100, 2000 .

[3] V. Bach and J. S. Møller. Correlation at low temperature: I. Exponential decay. J. Funct. Anal., 203: 93-149, 2003.

[4] V. Bach and J. S. Møller. Correlation at low temperature: II. Asymptotics. J. Stat. Phys., 116: 591-628, 2004 .

[5] D. Bao, S.-S. Chern, and Z. Shen. An introduction to Riemann-Finsler geometry. Graduate Texts in Mathematics, 200, Springer, New York, 2000.

[6] M. Giaquinta and S. Hildebrandt. Calculus of variations II. Grundlehren der mathematischen Wissenschaften, 311, Springer, Berlin-Heidelberg, 1996.

[7] B. Helffer and J. Sjöstrand. On the correlation for Kac like models in the convex case. J. Stat. Phys., 74: 349-409, 1994.

[8] M. Klein and E. Rosenberger. Agmon-type estimates for a class of difference operators. Preprint, mp arc 07-206, 2007.

[9] V. V. Kucherenko. Asymptotic solution of the Cauchy problem for equations with complex characteristics. J. Soviet math., 13: 24-81, 1980. Translated from Itogi Nauki $i$ Tekhniki, Sovremennye Problemy Matematiki, 8: 41-136, 1977.

[10] Y. Li and L. Nirenberg. The distance function to the boundary, Finsler geometry, and the singular set of viscosity solutions of some Hamilton-Jacobi equations. Comm. Pure Appl. Math., 58: 85-146, 2005.

[11] P. L. Lions. Generalized solutions of Hamilton-Jacobi equations. Research Notes in Mathematics, 69, Pitman, London, 1982.

[12] A. Martinez. An introduction to semiclassical and microlocal analysis. Universitext, Springer, New York-Berlin-Heidelberg, 2002.

[13] O. Matte. Supersymmetric Dirichlet operators, spectral gaps, and correlations. Annales Henri Poincaré, 7: 731-780, 2006.

[14] A. Melin and J. Sjöstrand. Fourier integral operators with complex-valued phase functions. In: Fourier integral operators and partial differential equations. Colloq. Internat., Univ. Nice, Nice, 1974, pp. 120-223. Lecture Notes in Mathematics, 459, Springer, Berlin, 1975.

[15] A. Melin and J. Sjöstrand. Fourier integral operators with complex phase functions and parametrix for an interior boundary value problem. Comm. Partial Differential Equations, 1: 313-400, 1976.

[16] A. Menikoff and J. Sjöstrand. On the eigenvalues of a class of hypoelliptic operators. Math. Ann., 235: 55-85, 1978.

[17] P. J. Paes-Leme. Ornstein-Zernike and analyticity properties for classical lattice spin systems. Ann. Phys., 115: 367-387, 1978. 
[18] E. Rosenberger. Asymptotic spectral analysis and tunnelling for a class of difference operators. Thesis, university of Potsdam. http://nbn-resolving.de/urn:nbn:de:kobv:517-opus-7393, 2006.

[19] J. Sjöstrand. Applications of Fourier distributions with complex phase functions. In: Fourier integral operators and partial differential equations. Colloq. Internat., Univ. Nice, Nice, 1974, pp. 255-282. Lecture Notes in Mathematics, 459, Springer, Berlin, 1975.

[20] J. Sjöstrand. Correlation asymptotics and Witten Laplacians. St. Petersburg Math. J., 8: 123-147, 1997.

[21] J. Sjöstrand. Complete asymptotics for correlations of Laplace integrals in the semi-classical limit. Mém. Soc. Math. France, 83, 2000.

[22] F. Treves. Introduction to pseudodifferential and Fourier integral operators II. Plenum Press, New York-London, 1980. 